В. И. БОБРОВСКИЙ, Д.Н. КОЗАЧЕНКО, Р. В. ВЕРНИГОРА, В. В. МАЛАШКИН

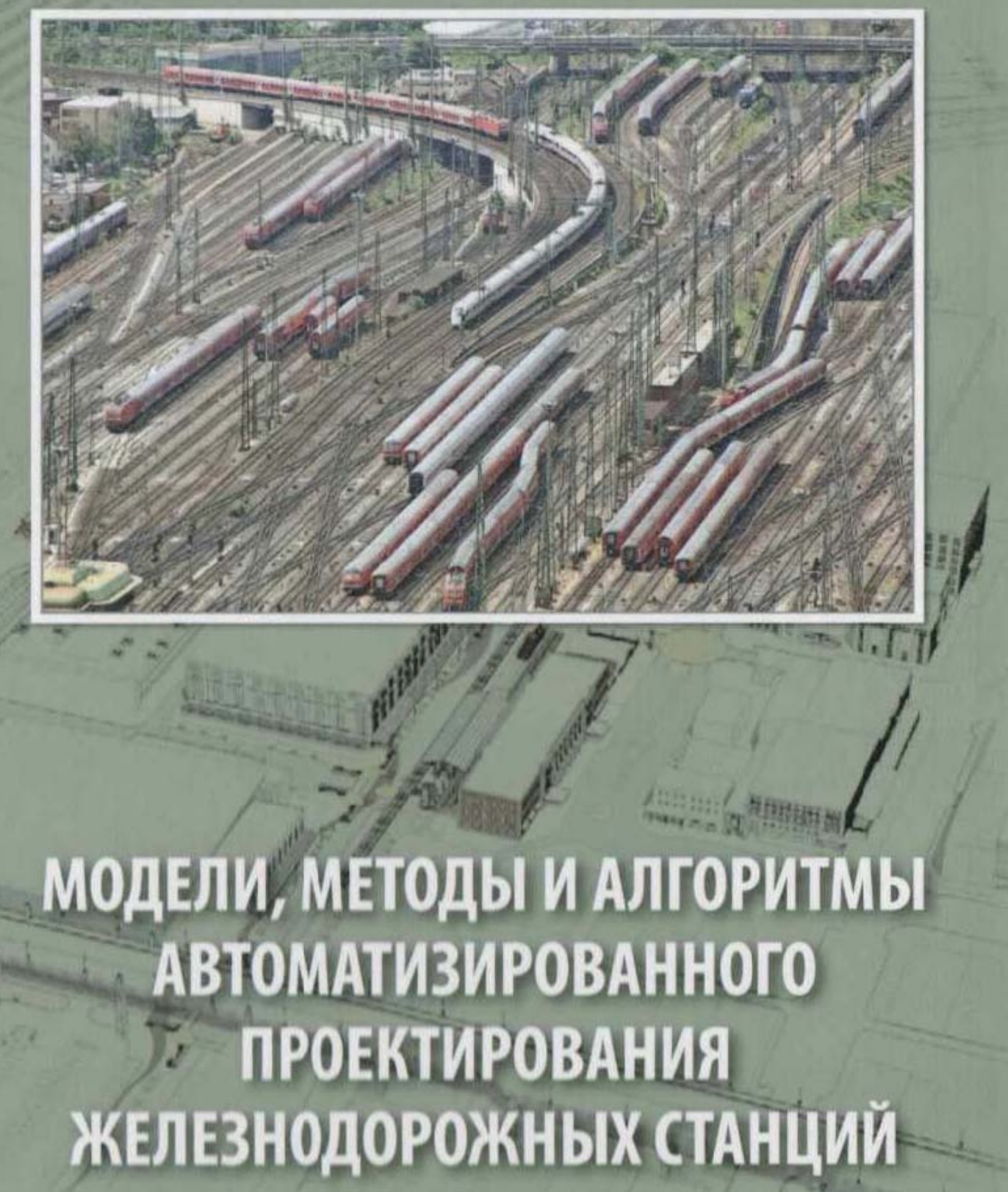




\section{МИНИСТЕРСТВО ТРАНСПОРТА И СВЯЗИ УКРАИНЫ}

Днепропетровский национальный университет железнодорожного транспорта имени академика В. Лазаряна

В.И. БОБРОВСКИЙ Д.Н. КОЗАЧЕНКО

Р.В. ВЕРНИГОРА

В.В. МАЛАШКИН

МОДЕЛИ, МЕТОДЫ И АЛГОРИТМЫ

АВТОМАТИЗИРОВАННОГО ПРОЕКТИРОВАНИЯ ЖЕЛЕЗНОДОРОЖНЫХ СТАНЦИЙ 
УДК 725.31

ББК 39.213-2я9

M 74

ISBN 978-966-1507-24-0

Издательство Маковецкий Ю.В.

Днепропетровск, 2010

Рекомендовано к печати решением Ученого совета Днепропетровского национального университета железнодорожного транспорта имени академика В. Лазаряна (протокол № 1 от 28.09.2009 года)

\section{Рецензенты:}

Д-р техн. наук, проф. Евгений Васильевич Нагорный, заведуюший кафедрой «Транспортные технологии» Харьковского наичонального автомобильно-дорожного университета, Украина

Д-р техн. наук, проф. Татьяна Васильевна Бутько, заведуюшая кафедрой «Управление эксплуатационной работой» Украинской государственной академии железнодорожного транспорта, г. Харьков, Украина

\section{МОДЕЛИ, МЕТОДЫ И АЛГОРИТМЫ АВТОМАТИЗИРОВАННОГО ПРОЕКТИРОВАНИЯ ЖЕЛЕЗНОДОРОЖНЫХ СТАНЦИЙ}

М 74 МОДЕЛИ, МЕТОДЫ И АЛГОРИТМЫ АВТОМАТИЗИРОВАННОГО ПРОЕКТИРОВАНИЯ ЖЕЛЕЗНОДОРОЖНЫХ СТАНЦИЙ: Монография / В.И. Бобровский, Д.Н. Козаченко, Р.В.

Вернигора, В.В. Малашкин - Дн-вск: Изд-во Маковецкий, 2010. - 156с. — ISBN 978-966-1507-24-0

УДК 725.31

ББК 39.213-2я9

Монография посвящена вопросам автоматизации проектирования путевого развития железнодорожных станций.

Книга предназначена для ученых, инженеров, аспирантов, студентов высших учебных заведений железнодорожного транспорта

(C) Издательство Маковецкий Ю.В. Днепропетровск, 2010

(C) Дніпропетровський Національний університет залізничного транспорту імені академіка В. Лазаряна, 2010 


\section{СОДЕРЖАНИЕ}

ВВЕДЕНИЕ

ГЛАВА 1 АНАЛИЗ СОВРЕМЕННОГО СОСТОЯНИЯ ПРОБЛЕМЫ АВТОМАТИЗАЦИИ ПРОЕКТИРОВАНИЯ ЖЕЛЕЗНОДОРОЖНЫХ

СТАНЦИЙ 8

ГЛАВА 2 СИСТЕМА ГЕОМЕТРИЧЕСКИХ МОДЕЛЕЙ ПЛАНА ПУТЕВОГО РАЗВИТИЯ СТАНЦИЙ 16

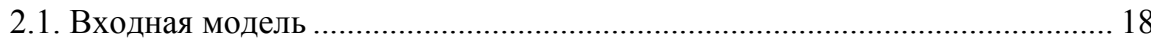

2.2. Внутренняя модель плана путевого развития станции .............................. 22

2.2.1. Каноническая модель плана станции ........................................... 22

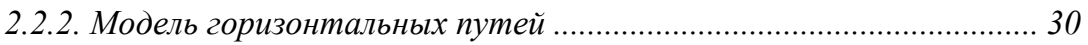

2.2.3. Представление сигналов во внутренней модели станции.............. 31

2.3. Внутренняя модель горочной горловины сортировочного парка............. 32

2.3.1. Каноническая модель спускной части горки.................................. 32

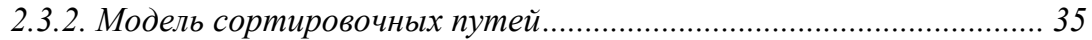

2.4. Выходные модели плана путевого развития станций ................................. 37

2.4.1. Расчетные параметры плана путевого развития......................... 37

2.4.2. Модель для интерактивного проектирования крупных станций 39

2.4.3. Модель для построения масштабных планов станций................... 41

ГЛАВА 3 АВТОМАТИЗИРОВАННОЕ ФОРМИРОВАНИЕ СИСТЕМЫ ГЕОМЕТРИЧЕКИХ МОДЕЛЕЙ ПЛАНА СТАНЦИИ …………………......... 44

3.1. Ввод немасштабной схемы станции и построение входной модели ........ 44

3.2. Формирование модели горизонтальных путей станции ............................ 48

3.3. Преобразование входной модели станции в каноническую......................5 52

3.3.1. Формирование списков инцидентности вершин орграфа станщии52

3.3.2. Определение направления боковых путей стрелочных переводов 53

3.3.3. Определение направления поворота круговых кривых .................. 57

3.3.4. Формирование списка сигналов на плане станщуи .......................... 58 


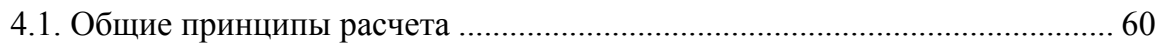

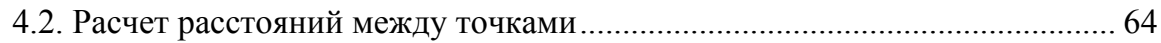

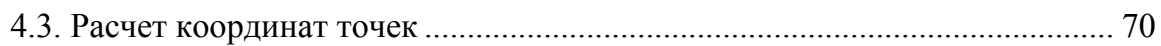

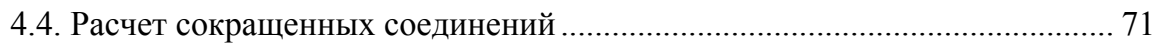

4.5. Расчет специализированных стрелочных улиц............................................ 75

4.5.1. Сокращенные стрелочные улищы .................................................. 75

4.5.2. Стрелочная улица под двойным углом крестовины ....................... 81

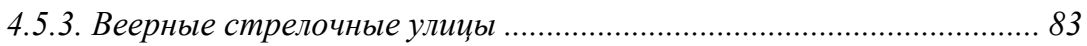

4.6. Объединение базовых элементов в общий план станции ........................... 87

4.7. Определение положения предельных столбиков и сигналов .................... 91

4.8. Расчет потребной полезной длины путей на станциях ............................. 97

4.8.1. Сущуность задачи и методика ее рещения ...................................... 97

4.8.2. Методика расчета полезных длин путей в отдельном парке ....... 99

4.8.3. Алгоритм комплексного расчета полезных длин путей станщии 101

ГЛАВА 5 РАСЧЕТ ГОРОЧНЫХ ГОРЛОВИН СОРТИРОВОЧНЫХ ПАРКОВ104

5.1. Расчет углов поворота дополнительных кривых ........................................ 104

5.2. Расчет элементов плана горочной горловины ………............................. 105

5.3. Проектирование конечных соединений сортировочных путей .............. 110

5.4. Определение положения замедлителей ….............................................. 113

5.5. Поиск точек пересечения эквидистант смежных сортировочных путей 115

ГЛАВА 6 ПРОГРАММНАЯ РЕАЛИЗАЦИЯ СИСТЕМЫ

АВТОМАТИЗИРОВАННОГО ПРОЕКТИРОВАНИЯ ПЛАНА ПУТЕВОГО

РАЗВИТИЯ СТАНЦИЙ................................................................................ 121

6.1. Общая характеристика программного обеспечения............................... 121

6.2. Программа графического ввода немасштабной схемы станции .............. 121 
6.3. Программа расчета координат точек плана путевого развития станции 125

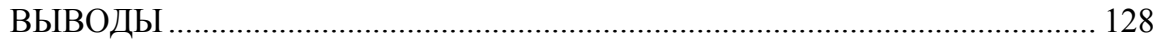

БИБЛИОГРАФИЧЕСКИЙ СПИСОК .............................................................. 131 ПРИЛОЖЕНИЕ А РАСЧЕТ ПЛАНА ПУТЕВОГО РАЗВИТИЯ СТАНЦИИ136 ПРИЛОЖЕНИЕ Б РАСЧЕТ ПЛАНА ГОРОЧНОЙ ГОРЛОВИНЫ ............... 141 ПРИЛОЖЕНИЕ В РАСЧЕТ ПЛАНА СОКРАЩЕННОЙ ГОРЛОВИНЫ .... 144 ПРИЛОЖЕНИЕ Г РАСЧЕТ ПЛАНОВ СТРЕЛОЧНЫХ УЛИЦ .................... 147 


\section{ВВЕДЕНИЕ}

Железнодорожные станции являются одним из главных элементов транспортной инфраструктуры и играют важную роль в обеспечении потребностей государства и населения в перевозках. В современных условиях, характеризующихся нестабильностью объемов перевозок, изменениями структуры и направления транспортных потоков, необходимостью сокращения эксплуатационных расходов железных дорог, основной целью совершенствования станций является приведение их конструкции и технологии в соответствие с объемами работы. Эффективным средством решения задачи поиска рациональных путей совершенствования конструкции, технического оснащения и технологии работы железнодорожных станций являются математические модели, методы и алгоритмы анализа и синтеза станций в сочетании с использованием современных средств вычислительной техники и информационных технологий.

Как показал выполненный анализ научных трудов, методы прямого синтеза оптимальной конструкции железнодорожных станций отсутствуют. Поэтому для поиска рационального варианта станции используется метод его последовательного улучшения, основанный на решении задач анализа и синтеза. Исходный вариант путевого развития станции представляется в виде немасштабной схемы, на базе которого ЭВМ осуществляет построение геометрических моделей и выполняет параметрический синтез плана станции. В результате синтеза получают масштабный план путевого развития станции, который необходим для формирования ее функциональной модели с целью анализа проекта на основе имитационного моделирования. Результаты моделирования используются для оценки варианта, анализа его недостатков и выбора путей совершенствования. Для реализации данной методики проектирования необходима интегрированная система структурных и функциональных моделей станций, построенная на единой методологической основе с использованием системного подхода. 
Для описания конструкции путевого развития станций использованы геометрические модели, основанные на взвешенных ориентированных графах. В работе представлена система геометрических моделей, используемых на отдельных этапах синтеза (входные, внутренние, выходные), а также методы их преобразования.

Разработанные модели и методы использованы при создании программного обеспечения для практической реализации автоматизированного проектирования железнодорожных станций.

Монография предназначена для ученых, инженеров, аспирантов и студентов высших учебных заведений железнодорожного транспорта. 


\section{ГЛАВА 1}

\section{АНАЛИЗ СОВРЕМЕННОГО СОСТОЯНИЯ ПРОБЛЕМЫ АВТОМАТИЗА- ЦИИ ПРОЕКТИРОВАНИЯ ЖЕЛЕЗНОДОРОЖНЫХ СТАНЦИЙ}

Пропускная и перерабатывающая способность железнодорожных станций и узлов, эффективность их эксплуатации напрямую зависят от оптимальности решений, принятых при их проектировании. Существенное повышение качества проектирования, увеличение производительности труда проектировщиков может быть достигнуто в результате внедрения новой информационной технологии, основой которой является система автоматизированного проектирования (САПР). Одной из основных проблем теории САПР является разработка эффективных математических моделей проектируемых объектов и алгоритмов выполнения проектных процедур. Эта задача является особенно важной для проектирования железнодорожных станций и узлов, что объясняется высокой стоимостью их строительства и реконструкции, длительным сроком эксплуатации, невозможностью построения физических моделей.

При проектировании железнодорожных станций и узлов наиболее массовыми и трудоемкими являются расчеты соединений путей, что обусловило разработку программ и использование ЭВМ для выполнения этих расчетов. Исследования и алгоритмизация методов расчета соединений путей начаты в 60-х годах одновременно с началом широкого внедрения ЭВМ в практику инженерно-технических расчетов. Во многих организациях (Мосгипротранс, ЦНИИС, Ленгипротранс и др.) были разработаны программы $[1,2]$, обеспечивающие выполнение на ЭВМ расчетов соединений путей разного типа. Одновременно выполняются первые научные исследования, в которых разрабатываются методики и алгоритмы расчетов соединений путей [3-4]. Среди указанных программ были специализированные, предназначенные для расчета отдельных элементов соединений путей, а также универсальные, позво- 
ляющие рассчитывать комплексы взаимосвязанных элементов любой структуры.

В рассматриваемой задаче существует две основные проблемы: формализация схемы станции и ее исходных параметров для ввода в ЭВМ (построение модели станции) и разработка алгоритма анализа модели для расчета выходных параметров.

В ЦНИИСе [3] выполнен детальный логический анализ двух вариантов формализации схем станций - кодирование схемы размещения элементов путевого развития станций и кодирование уравнений, необходимых для ее расчета. В результате анализа был сделан выбор в пользу второго варианта, который обеспечивал большую надежность расчетов, простоту алгоритма и универсальность программы, что при имеющихся в то время технических средствах было немаловажным. В тоже время отмечено, что во втором варианте степень автоматизации расчетов ниже, поскольку последовательность расчетов устанавливается проектировщиком.

В дальнейшем на базе указанных принципов ЦНИИСом и Мосгипротрансом разработана методика, алгоритм и программа для расчетов соединений путей на ЭВМ [5]. Указанная программа состоит из управляющего блока, не зависящего от схемы станции, и библиотеки модулей для расчета отдельных типовых соединений путей. Проектировщик определяет порядок расчета схемы, выбирает необходимые модули, задает по определённому макету требуемые числовые параметры для каждого из них (линейные и угловые), а также обеспечивает передачу промежуточных (расчетных) параметров между модулями. Теоретически программа обеспечивает расчет любой конструкции соединений путей; практически существуют ограничения по наличию необходимых модулей в библиотеке и по памяти ЭВМ. Важным достоинством указанной методики является возможность задания ограничений на решение и их контроль, многовариантные и оптимизационные расчеты определенных элементов схем, что особенно важно в стесненных условиях и при реконструкции станций, а также автоматизированный подбор варьи- 
руемых исходных данных для получения наилучшего решения. Существенным недостатком указанной методики является высокая сложность подготовки данных для расчетов, что существенно затрудняет использование разработанной программы.

Методические вопросы автоматизации расчетов плана путевого развития парков станций, расположенных на кривых, рассматриваются в [6].

Фундаментальные исследования проблемы автоматизации расчетов путевого развития станций были выполнены в ИК АН УССР [7, 4]. В указанных работах впервые был разработан метод кодирования схем станций с использованием теории графов. При этом конструкция любой схемы представляется множеством элементарных циклов (контуров), выделяемых на графе схемы. Разработан также алгоритм расчета координат схемы путевого развития станции, основанный на предварительных расчетах и увязке параметров элементарных циклов схемы. Разработанная контурная модель станции [4] является универсальной формой представления информации о ее схеме. Указанная модель после автоматического преобразования может быть использована для решения широкого класса задач проектирования станций, в частности для расчета пропускной способности горловин [8]. К недостаткам контурного представления схем следует отнести избыточность, так как при этом каждое ребро графа, как правило, включается в модель дважды. Как отмечено в [4], данная модель станции позволяет существенно упростить алгоритм переработки исходной информации ценой возможных ограничений по памяти ЭВМ. На наш взгляд, главным недостатком контурной модели является сложность ее подготовки (выделение контуров на схеме станций) и большой избыточный объем вводимой информации, поскольку указанные операции выполняются вручную. Достаточно сложно также корректировать контурную модель при необходимости внесения изменений в схему станции. В этой связи целесообразно упрощение входной модели станции, возможно, за счет усложнения алгоритма ее преобразования и анализа. 
Возрастание сложности проектируемых станций и узлов, необходимость повышения производительности труда проектировщиков, качества и обоснованности проектных решений с одной стороны, а также развитие математических методов и технических средств (ЭВМ) с другой стороны, сделали актуальной задачу создания системы автоматизированного проектирования станций $[9,10]$. В данных работах перечислены основные предпосылки для создания САПР ЖС, подчеркивается необходимость развития методов имитационного моделирования транспортных объектов для решения широкого класса задач проектирования станций, в том числе и оптимизационных задач. В $[10,11]$ предложена возможная структура построения САПР, а также перечислены основные этапы ее создания. В частности, в качестве этапов развития САПР и ее составных частей выделены задачи автоматизации расчетов соединений путей (первый этап) и моделирования транспортных систем с целью их анализа и синтеза (второй этап). Указанные этапы могут послужить основой технологической линии проектирования (ТЛП) железнодорожных станций и узлов.

Вопросы формализации схем станций для автоматизации расчетов и проектирования рассматриваются в работах, выполненных в МИИТе [12-15]. Разработан метод формализованного представления схем станций в виде двухуровневых структур, содержащих информацию о секциях станции и элементах каждой секции [15]. Использован табличный метод кодирования схем; предусмотрено четыре вида таблиц разной структуры, включающих информацию как о топологии схемы, так и о ее параметрах. Необходимо отметить системный подход к проектированию в указанных работах, в которых упоминается комплексная система, позволяющая выполнить расчет координат точек станции, построение ее масштабного плана, а также рассчитывать технологические показатели на основе имитационного моделирования. Указывается, что данная система позволяет проектировщику в диалоговом режиме анализировать введенные в ЭВМ варианты схем станций и на этой основе выбирать вариант для реализации. К недостаткам следует отнести сложность табличного метода кодирования схем станций вообще и использо- 
ванной в [15] структуры в частности, а также необходимость ручной подготовки и ввода.

Методика формализации конструктивных параметров железнодорожных станций с целью получения на ЭВМ их графического изображения приведена в [16]. Станция представляется как блочно-иерархическая система, в которой выделено три уровня - функциональный, структурный и уровень базисных элементов. Входная информация о станции представлена в виде таблиц шести различных форм, содержащих данные о топологии схемы, о путях станции, о типе и параметрах стрелочных улиц, о поворотах станционных путей и параметрах соответствующих кривых. В результате расчетов определяются координаты точек плана и все необходимые данные для построения на графопостроителе чертежа станции. В указанной работе отмечены также другие возможные сферы использования предложенного метода формализации схем - моделирование работы станций для получения техникоэксплуатационных показателей, а также создание информационных систем для хранения данных о планах станций, их техническом оснащении и показателях эксплуатационной работы. Недостаток работы - высокая сложность и трудоемкость работ по подготовке и вводу в ЭВМ формализованных данных о схеме станции. Одной из весьма трудоемких процедур, выполняемых при проектировании планов путевого развития станций, является расстановка предельных столбиков (ПС) и сигналов. В настоящее время имеется ряд специальных программ $[1,17]$ для автоматизации решения данной задачи. Однако, как показывает анализ, несовершенство использованных моделей путевого развития и алгоритмов распознавания ситуаций вызывает необходимость выполнения проектировщиком значительной подготовительной работы, что ограничивает область применения указанных программ. Поэтому возникает задача автоматизации расчетов координат ПС и сигналов для всех возможных случаев их установки при минимальной входной информации.

Для успеха автоматизированного проектирования станций важно установить показатели, характеризующие качество конструкции их путевого раз- 
вития. В этой связи большой интерес представляют работы $[18,19]$, в которых предлагается минимизировать удельную строительную длину путей, энергетические расходы на движение поездов, а также расходы на ремонт путей и подвижного состава за счет выбора рациональной конструкции горловин станции.

Существенные особенности имеет проектирование горочных горловин сортировочных парков, при котором необходимо обеспечить размещение стрелочных переводов, тормозных позиций, а также устройств автоматики с учетом допускаемых радиусов кривых, ширины междупутий и других технических условий. Кроме того, при проектировании необходимо определить положение и параметры дополнительных кривых на спускной части горки и на сортировочных путях. Для решения указанной задачи на ЭВМ трасса расчетного пути задается уравнением проекций отдельных участков трассы на вертикальную ось [20]. Принципиальная сложность данного расчета заключается в том, что в указанное уравнение обычно входит несколько в общем случае неизвестных углов. Поэтому для решения уравнения приходится принимать значения некоторых углов подбором на основе предварительного приближенного решения задачи графическим методом [21], либо по данным проектов аналогичных горловин. Недостатком указанного способа, кроме его трудоемкости, является сложность формализации структуры уравнения и задания его параметров до расчета. Учитывая указанную неопределенность, в [22] была предложена методика оптимизации проектирования трассы расчетного крайнего пути по критерию его минимальной расчетной длины. Была сформулирована условная вариационная задача, для решения которой использован метод множителей Лагранжа; в результате были получены все неизвестные углы расчетного пути. Данный метод, однако, не получил распространения, так как в нем не учитываются ограничения, накладываемые на значения отдельных углов, а также их влияние на условия вписывания внутренних пучков горловины. Кроме того, как показывают исследования, сумма 
углов поворота расчетного пути и его расчетная длина при вариации отдельных углов изменяются весьма незначительно.

Существующая технология проектирования станций ориентирована на работу с чертежом. Чертеж является носителем информации для воспроизведения запроектированного объекта. Однако этим роль чертежа не исчерпывается. Построение и коррекция геометрической модели станции представляет собой итерационный процесс, распределенный по всем этапам проектирования. В этих условиях проектировщику необходим внешний накопитель информации, адекватно отображающий объект. Таким накопителем выступает чертеж, эскиз или набросок. Чертеж имеет большую емкость и обеспечивает высокую скорость поиска и выбора необходимой информации. Процесс взаимодействия проектировщика с геометрической моделью с помощью чертежа является одним из важнейших, облегчающих принятие решения.

Таким образом, традиционную методику проектирования можно представить в виде схемы, изображенной на рис. 1.1.

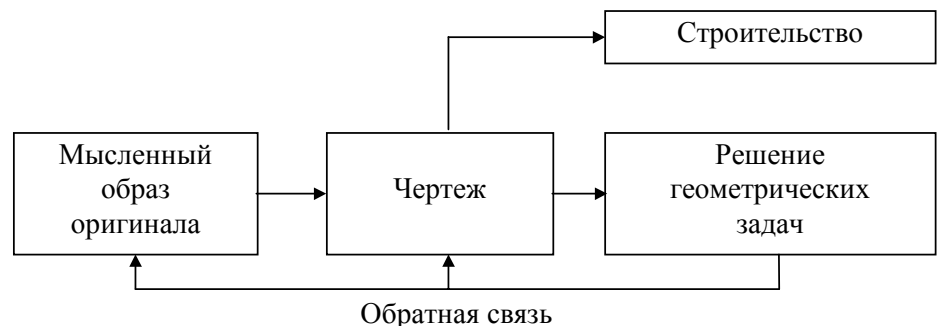

Рисунок 1.1 - Схема традиционной технологии проектирования

При автоматизированном проектировании все или часть проектных решений получают в результате взаимодействия человека и ЭВМ. САПР должна усиливать творческие способности человека за счет возможности современных ЭВМ быстро перерабатывать большие объемы информации, в том числе графической. Автоматизированное проектирование основывается на математической модели объекта (см. рис. 1.2). 


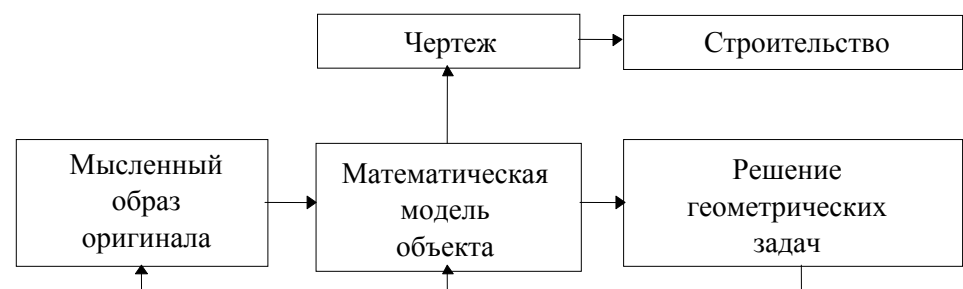

Обратная связь

Рисунок 1.2 - Схема автоматизированного проектирования

Математическая модель является более совершенным способом представления планов путевого развития станций и более мощным и удобным инструментом для его технико-эксплуатационной оценки. Чертеж в этих условиях начинает играть вспомогательную роль, а построение модели выполняется либо автоматически, либо автоматизированно с помощью средств компьютерной графики. В этой связи одной из задач исследования является автоматизация построения математических моделей путевого развития станций на базе немасштабных схем, предлагаемых проектировщиком, с последующим их преобразованием в рабочие чертежи, сопровождаемые значениями всех числовых параметров плана.

Построение математической модели станции и разработка методов и алгоритмов расчета ее параметров позволит существенно ускорить процесс проектирования станций за счет использования графического ввода немасштабных схем, интерактивного режима работы с визуализацией результатов, автоматического расчета всех необходимых параметров путевого развития и построения рабочих чертежей. Кроме того, геометрическая модель станции, полученная при решении данной задачи, может быть использована и при функциональном моделировании станции, выполняемом для оценки качества проекта. Наконец, решение указанной задачи является обязательным шагом на пути к созданию интеллектуальной САПР ЖС. 


\section{ГЛАВА 2 \\ СИСТЕМА ГЕОМЕТРИЧЕСКИХ МОДЕЛЕЙ ПЛАНА ПУТЕВОГО РАЗВИТИЯ СТАНЦИЙ}

Анализ математических моделей путевого развития железнодорожных станций показал, что методы прямого синтеза его оптимальной конструкции в настоящее время развиты недостаточно. В связи с этим система моделей должна поддерживать итерационный процесс совершенствования исходного варианта станции на основе последовательного многократного решения задач анализа и синтеза.

В данной главе представлены геометрические модели станций (ГМ), используемые для обеспечения графического ввода в ЭВМ их немасштабных схем, автоматизации параметрического синтеза планов путевого развития, построения чертежей и подготовки данных для функционального моделирования.

Для решения задачи синтеза планов путевого развития станций необходима система геометрических моделей, используемых на отдельных его этапах (входные, внутренние, выходные), а также методы их преобразования. В основу моделей положено представление схем станций в виде орграфов, вершинам и дугам которых ставятся в соответствие необходимые параметры.

Входная модель станции должна обеспечить возможность графического ввода немасштабной схемы с его визуальным контролем, который позволяет избежать трудоемкого ручного кодирования и исключить появление ошибок. Графический ввод должен дополняться параметризацией отдельных элементов, которая выполняется с использованием принципа умолчания (вместо нерегламентированных параметров используются их наиболее вероятные значения - марки стрелочных переводов, радиусы кривых, вставки и т.д.). Входная модель должна обеспечивать возможность ее автоматического преобразования во внутреннюю модель станции. 
Внутренняя модель станции должна отображать состав элементов станции (пути, стрелочные переводы, соединительные кривые, предельные столбики, сигналы и др.), их взаимное расположение и взаимосвязь, а также необходимые размеры. Данная модель предназначена для автоматического расчета основных параметров путевого развития; она включает каноническую модель станции и модель ее горизонтальных путей и используется для расчета плана путевого развития.

Каноническая модель отображает топологическую структуру станции и может быть представлена в виде взвешенного орграфа. Вершинам орграфа ставятся в соответствие характерные точки плана путевого развития станции, а каждая его дуга соответствует участку пути между указанными точками. Горизонтальные пути станции представлены вершинами древовидного графа, ребрам которого поставлены в соответствие заданные междупутья.

По окончании расчетов внутренняя модель преобразуется в выходную, отображающую не только взаимное расположение элементов путевого развития станции в пространстве, но и их геометрическую форму и размеры. Выходная ГМ предназначена для визуализации результатов расчета плана в интерактивном режиме, а также для построения чертежей. Кроме того, на базе данных выходной ГМ определяются строительные и полные длины путей, с помощью которых могут быть определены показатели качества проекта. Выходная модель может служить основой для решения широкого круга задач, в том числе и для функционального моделирования станций с целью получения их технико-технологической оценки.

В целом интегрированное семейство ГМ должно обеспечить итерационный процесс синтеза рациональных планов станций с заданными свойствами и наилучшими характеристиками [23]. С этой целью должна быть предусмотрена возможность интерактивного изменения моделей; при этом редактирование схемы станции (структурный синтез) выполняется на уровне входной ГМ, а отдельных ее параметров (параметрический синтез) - на уровне внутренней ГМ. Указанные модели должны обладать универсальностью, позволяя выполнять конструирование и расчет станций, а также по- 
строение их масштабных планов для анализа работы с помощью методов имитационного моделирования.

В работе приведены структура и основные принципы построения семейства ГМ планов путевого развития станций, а также методика их использования для решения сформулированных задач; теоретические основы геометрического моделирования планов станций на разных его этапах проиллюстрированы на примере станции А, схема которой приведена на рис. 2.1.

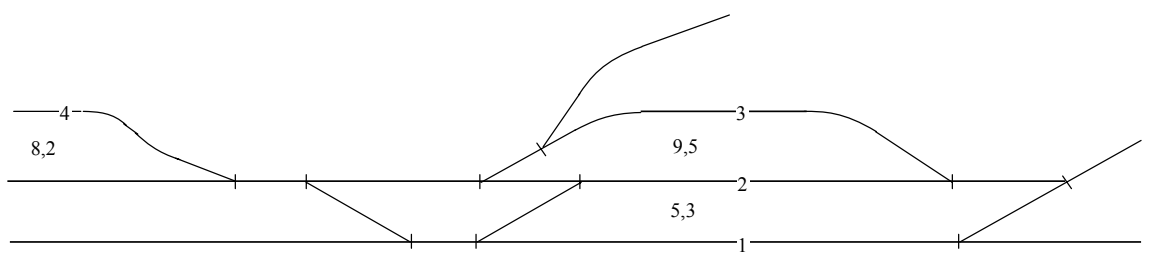

Рисунок 2.1 - Схема путевого развития станции А

\section{1. Входная модель}

Разработка схемы станции и создание ее входной модели характеризуется наибольшим уровнем взаимодействия проектировщика и ЭВМ. Поэтому на этапе ввода в ЭВМ схемы путевого развития железнодорожной станции структура ее математической модели должна быть основой для построения эффективного графического редактора, ориентированного на решение этой прикладной задачи.

На данном этапе схема путевого развития представляется в виде множества графических объектов $\Omega_{\text {вх }}$. При этом выделены следующие типы объектов: участок пути е, центр стрелочного перевода $\mathbf{s}$, вершина угла поворота c, номер пути w, междупутье $\mathbf{m}$, сигнал $\mathbf{l}$. Каждому объекту ставится в соответствие тип, экранные координаты характерных точек $\mathbf{p}=(x, y)$ и список конструктивных параметров.

Участок пути (объект LINE) описывается следующей структурой:

$$
\mathbf{e}=\left(\mathbf{p}_{e \mathrm{H}}, \mathbf{p}_{e \kappa}, \mu_{e}, l\right),
$$

где $\mathbf{p}_{e \mathrm{H}}, \mathbf{p}_{\text {eк }}-$ начальная и конечная точки отрезка; 
$\mu_{e}-$ метод определения длины участка е;

$l$ - параметр, характеризующий длину участка е.

Длина участка пути определяется в соответствии с установленным методом $\mu_{e}\left(\mu_{e} \in[0,6]\right)$ :

0 - длина определяется автоматически на основе анализа схемы;

1 - длина определяется по разности координат смежных точек;

2 - длина определяется численным значением $l$;

3 - длина определяется шириной междупутья $l$;

4, 5, 6 - длина определяется из условия обеспечения полезной длины $l$ данного пути парка, соответственно в четном, нечетном и в обоих направлениях.

Центры стрелочных переводов (объекты SWITCH) и вершины углов поворота кривых (объекты CURVE) описываются структурами:

$$
\begin{gathered}
\mathbf{s}=\left\{\mathbf{p}_{S}, N_{S}, m_{S}, T_{S}, c_{S}\right\}, \\
\mathbf{c}=\left\{\mathbf{p}_{C}, N_{C}, r_{C}, \boldsymbol{\mu}_{C}, \alpha_{C}, N_{T}\right\},
\end{gathered}
$$

где $\mathbf{p}_{S}, \mathbf{p}_{C}-$ экранные координаты, соответственно, центра стрелочного перевода и вершины угла поворота;

$N_{S}, N_{C}-$ соответственно, номер стрелочного перевода и вершины угла поворота кривой;

$m_{S}-$ марка крестовины;

$T_{S}$ - тип рельса;

$c_{S}$ - наличие электрической централизации;

$\mu_{C}-$ метод определения угла (0 - определяется автоматически на основе анализа схемы; 1 - задано численное значение; 2 - сокращенное соединение);

$\alpha_{C}-$ величина угла поворота;

$N_{T}$ - номер расчетной вершины при расчете параметров конечного соединения. 
Номера путей и междупутья (объекты, соответственно, WAY и MIDWAY) представляются структурами.

$$
\begin{aligned}
& \mathbf{w}=\left\{\mathbf{p}_{w}, N_{w}\right\}, \\
& \mathbf{m}=\left\{\mathbf{p}_{m}, g_{m}\right\},
\end{aligned}
$$

где $\mathbf{p}_{w}, \mathbf{p}_{m}-$ координаты точки вставки номера пути и междупутья;

$N_{w}$ - номер пути;

$g_{m}$ - ширина междупутья.

Сигналы (объекты SIGNAL) описываются структурой

$$
\mathbf{q}_{\mathrm{Bx}}=\left\{\mathbf{p}_{q}, N_{q}, d_{q}, k_{q}\right\},
$$

где $\mathbf{p}_{q}-$ координаты вставки сигнала;

$N_{q}$ - номер сигнала;

$d_{q}$ - направление действия сигнала $(0$ - в направлении, противоположном направлению ориентации дуг, 1 - в направлении ориентации дуг);

$k_{q}$ - тип сигнала $(0$ - мачтовый, 1 - карликовый, 2 - карликовый спаренный и др.).

В памяти ЭВМ каждый графический объект представляются с помощью ассоциативного списка

$$
\left(\left(a_{1} . t_{1}\right)\left(a_{2} . t_{2}\right) \ldots\left(a_{N} \cdot t_{N}\right)\right),
$$

где $a_{1} \ldots a_{N}-$ ключи, представляющие собой цифровой код параметров, входящих в структуры (2.1)-(2.5) (см. табл. 2.1);

$t_{1} \ldots t_{N}$ - связанные с ключами данные.

В целом математическая модель $\Omega_{\mathrm{Bx}}$ представляет собой список графических объектов, которые записываются в порядке ввода схемы в ЭВМ.

На рис. 2.2 приведен фрагмент схемы станции и соответствующий ему список $\Omega_{\text {вх }}$ Созданная входная модель может быть сохранена в виде текстового файла в формате stn; в Приложении А (см. табл. А.1) приведен файл входной модели станции А (см. рис. 2.1). 
Таблица 2.1 - Список цифровых кодов параметров объектов входной модели

\begin{tabular}{|l|l|l|l|}
\hline Ключ & Параметр & Ключ & Параметр \\
\hline 0 & Тип объекта & 31 & $\mu_{C}$ \\
\hline 1 & $N_{S}, N_{C}, N_{w}, N_{r}$ & 32 & $\mid \alpha_{C}$ \\
\hline 10 & $\begin{array}{l}\text { Координаты точек } \mathbf{p}_{e н}, \mathbf{p}_{s}, \\
\mathbf{p}_{c}, \mathbf{p}_{w}, \mathbf{p}_{m}, \mathbf{p}_{r}\end{array}$ & 33 & $N_{T}$ \\
\hline 11 & Координаты точки $\mathbf{p}_{e \kappa}$ & 40 & $\mu_{e}$ \\
\hline 20 & $m_{S}$ & 41 & $L$ \\
\hline 21 & $T_{S}$ & 50 & $G$ \\
\hline 22 & $c_{S}$ & 60 & $d_{r}$ \\
\hline 30 & $r_{C}$ & 61 & $k_{r}$ \\
\hline
\end{tabular}

a)

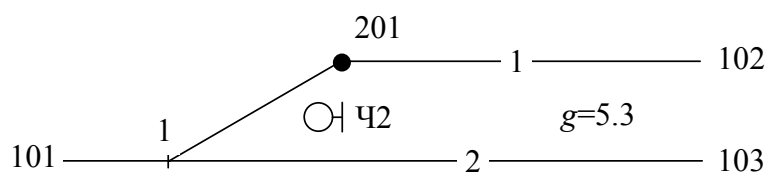

б)

\begin{tabular}{l|l}
\hline \multicolumn{1}{c|}{ Список } & \multicolumn{1}{c}{ Примечание } \\
\hline$(((0$ SWITCH) (1 1) (10 5 5) (20 0) (21 0) (22 1)) & Центр перевода 1 \\
\hline$((0$ CURVE) (1 201) (10 20 15)(30 300)(31 0) (32 0)) & Вершина угла поворота 201 \\
\hline$((0$ LINE) (10 0 5) (11 5 5)(40 0)(41 0)) & Отрезок 101-1 \\
\hline$((0$ LINE) (10 5 5) (11 50 5)(40 0)(41 0)) & 0трезок 1-103 \\
\hline$((0$ LINE) (10 5 5) (11 20 15)(40 0)(41 0)) & 0трезок 1-201 \\
\hline$((0$ LINE) (10 20 15) (11 50 15)(40 0)(41 0)) & 0трезок 201-102 \\
\hline$((0$ WAY) (1 1) (10 30 15)) & Номер пути 1 \\
\hline$((0$ WAY) (1 2) (10 25 5)) & Номер пути 2 \\
\hline$((0$ MIDWAY) (10 40 10)(50 5.3)) & Междупутье 2-1 \\
\hline$((0$ SIGNAL) (1 ч2) (10 20 10) (60 0) (61 0))) & Сигнал Ч2 \\
\hline
\end{tabular}

Рисунок 2.2 - Входная модель станции: $a$ - схема путевого развития; $\sigma$ - список графических объектов $\Omega_{\mathrm{Bx}}$ 


\section{2. Внутренняя модель плана путевого развития станции}

\subsection{1. Каноническая модель плана станици}

Каноническая модель плана путевого развития станции должна обеспечить:

- входной контроль введенной информации;

- возможность изменения отдельных параметров схемы, заданных по умолчанию;

- анализ, автоматическое распознавание типовых элементов схемы путевого развития и выбор программных модулей для расчета;

- комплексный расчет плана путевого развития и построение выходной модели.

Как показал анализ типов структурных моделей, для реализации процедур автоматизированного синтеза путевого развития станций наиболее подходящими являются ГМ, основанные на взвешенных орграфах. В состав канонической модели станции должны входить топологическая и параметрическая модели. Топологическая модель отображает состав элементов станции (пути, стрелочные переводы, соединительные кривые и др.), их взаимное расположение и взаимосвязь. Параметрическая модель содержит сведения о форме и геометрических размерах соответствующих элементов.

Топологическая модель станции представляет собой ориентированный граф $G=(V, E)$, в котором выделено три подмножества вершин: $V^{S}, V^{C}$ и $V^{W}$. Вершины $v_{i} \in V^{S}$ являются центрами стрелочных переводов (ЦП), вершины $v_{j} \in V^{C}-$ вершинами углов поворота кривых (ВУП), вершины $v_{k} \in V^{W}-$ концами путей (КП). Дуга графа $e=(v, u)$ обозначается упорядоченной парой, состоящей из начальной $v$ и конечной $u$ вершин; ее направление задано от вершины $v$ к вершине $u$. Принято, что все дуги ориентированы слева направо (см. рис. 2.3). 


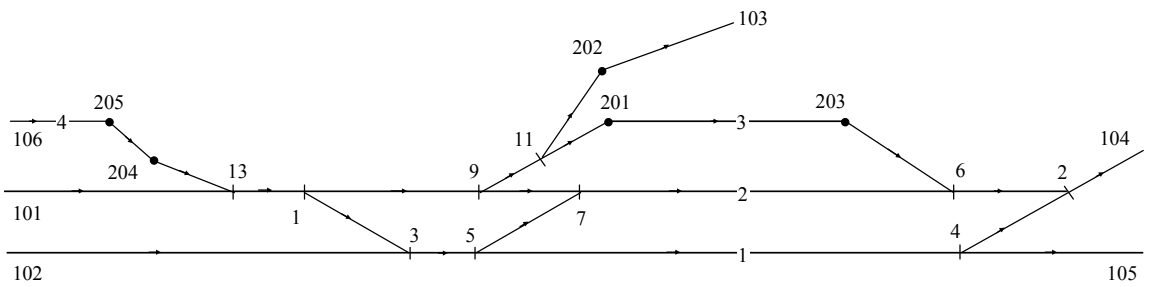

Рисунок 2.3 - Орграф $G=(V, E)$ схемы станции А

Степень вершины ориентированного графа $\operatorname{deg} v$ определяется числом инцидентных ей дуг:

$$
\operatorname{deg} v=d^{+}(v)+d^{-}(v)
$$

Здесь $d^{+}(v), d^{-}(v)$ - соответственно полустепени исхода и захода вершины $v$, которые определяются как множество всех дуг, исходящих из вершины $v$ и заходящих в нее:

$$
d^{+}(v)=|\Gamma(v)| \quad \text { и } \quad d^{-}(v)=\left|\Gamma^{-1}(v)\right|,
$$

где $\Gamma(v)-$ множество вершин, являющихся конечными вершинами дуг, у которых начальной вершиной является $v$;

$\Gamma^{-1}(v)$ - множество вершин, являющихся начальными вершинами дуг, у которых конечной вершиной является $v$.

Полустепени вершин $v_{i} \in V^{S}$ ориентированного графа $G$ позволяют идентифицировать тип вершины (ЦП $-\operatorname{deg} v=3$, ВУ $-\operatorname{deg} v=2$, КП $\operatorname{deg} v=1)$, а также определить направление укладки стрелочных переводов $\left(\right.$ если $d^{+}(v)=2$ и $d^{-}(v)=1-$ перевод противошерстный; если $d^{+}(v)=1$ и $d^{-}(v)$ $=2$ - перевод пошерстный). При этом очевидно, что максимальная полустепень каждой вершины не превышает двух.

Из теории графов известно, что сумма полустепеней исхода всех вершин графа равна сумме полустепеней захода и равна числу его дуг [24]:

$$
\sum_{i=1}^{n} d^{+}\left(v_{i}\right)=\sum_{i=1}^{n} d^{-}\left(v_{i}\right)=m,
$$

где $n, m-$ соответственно число вершин и дуг графа $G$. 
Данное утверждение используется для входного контроля данных о схеме станции.

Для представления ориентированного графа станции в ЭВМ принята структура данных, называемая списками инцидентности [25]. Данная структура является наиболее экономичной в отношении памяти, т. к. рассматриваемый граф является неплотным $\left(m<<n^{2}\right)$. Списки инцидентности содержат для каждой вершины $v \in V$ список вершин $u$ таких, что $v \rightarrow u$. При этом, поскольку полустепень исхода каждой вершины графа $G$ не превышает двух, то для представления каждого списка достаточно двух переменных в памяти ЭВМ. В целом число переменных, необходимое для представления графа с помощью списков инцидентности, не превышает $3 n$.

Для разделения множества вершин графа $G$ на подмножества $V^{S}, V^{C}$, $V^{W}$, каждому из них выделены непересекающиеся группы номеров: $N^{S}=\{1,2$, $\ldots, 99\}, N^{W}=\{101,102, \ldots, 199\}, N^{C}=\{201,202, \ldots, 299\}$.

Ниже приведен пример списков инцидентности вершин $v_{i} \in V$ графа $G$, показанного на рис. 2.3 (см. табл. 2.2).

Таблица 2.2 - Списки инцидентности вершин орграфа $G$

\begin{tabular}{|r|r|r|r|r|r|}
\hline \multicolumn{1}{|c|}{$N$} & \multicolumn{1}{|c|}{$N_{P}$} & \multicolumn{1}{|c|}{$N_{B}$} & \multicolumn{1}{c|}{$N$} & \multicolumn{1}{c|}{$N_{P}$} & \multicolumn{1}{c|}{$N_{B}$} \\
\hline 1 & 9 & 3 & 202 & 103 & 0 \\
\hline 3 & 5 & 0 & 203 & 6 & 0 \\
\hline 5 & 4 & 7 & 204 & 13 & 0 \\
\hline 7 & +6 & 0 & 205 & 204 & 0 \\
\hline 9 & +7 & 11 & 101 & +13 & 0 \\
\hline 11 & 201 & 202 & 102 & +3 & 0 \\
\hline 13 & 1 & 0 & 103 & 0 & 0 \\
\hline 2 & 104 & 0 & 104 & 0 & 0 \\
\hline 4 & 105 & +2 & 105 & 0 & 0 \\
\hline 6 & 2 & 0 & 106 & 205 & 0 \\
\hline 201 & 203 & 0 & & & \\
\hline
\end{tabular}

Данный граф представлен матрицей размером $3 \times n(n=21)$; первый столбец матрицы представляет собой список всех вершин графа; в строках двух других столбцов - списки инцидентности соответствующих вершин. Длина каждого списка равна 0, 1 или 2 элемента. 
Для удобства анализа схем станций принято, что первым в списке для вершин $v_{i} \in V^{S}$ с полустепенью исхода $d^{+}\left(v_{i}\right)=2$ (противошерстные переводы) указывается номер вершины $u_{1}$, с которой данная стрелка (вершина $v_{i}$ ) связана по прямому пути (обыкновенный перевод) или правому пути (симметричный перевод); вторым в списке указывается номер другой вершины $u_{2}$, смежной с $v_{i}$ (например, на рис. $2.3 v_{1}=1, u_{1}=9, u_{2}=3$ ).

Для вершин $v_{i} \in V^{C}$ с полустепенью исхода $d^{+}\left(v_{i}\right)=1$ (ВУП, ЦП пошерстных стрелок) второй элемент списка отсутствует (рис. 2.3, $v_{4}=7, u_{1}=6$, $u_{2}=0$ ); для вершин $v_{i} \in V^{W}$ с полустепенью исхода $d^{+}\left(v_{i}\right)=0$ (КП) отсутствуют оба элемента списка $\left(v_{18}=103, u_{1}=0, u_{2}=0\right)$.

Следует заметить, что при принятом способе представления орграфа $G$ для некоторых вершин $v_{i} \in V^{S}, d^{+}\left(v_{i}\right)=1$ (ЦП пошерстных стрелок) невозможно определить из модели углы наклона смежных отрезков. Поэтому при наличии входной модели, эти углы устанавливаются на основании экранных координат вершин отрезков, а при табличном вводе внутренней модели в списках инцидентности необходимо конечные вершины дуг, заходящих в такие ЦП по прямому пути, пометить знаком '+'. Например, для ЦП 13 (см. рис. 2.3) конечная вершина 13 дуги 101-13 помечена знаком '+' (см. табл. 2.2); для ЦП 2 знаком ``` помечена конечная вершина дуги 4-2.

Орграф схемы станции $G=(V, E)$ является взвешенным; каждая вершина подмножеств $V^{S}, V^{C}, V^{W}$ характеризуется некоторым вектором параметров (соответственно, $\mathbf{X}^{S}, \mathbf{X}^{C}, \mathbf{X}^{W}$ ). В этой связи списки инцидентности вершин орграфа $G$ дополняются совокупностями соответствующих параметров $\mathbf{X}$. Окончательный вид канонической модели для схемы, показанной на рис. 2.3, приведен в табл. 2.3. Ниже рассмотрены особенности определения векторов параметров $\mathbf{X}$ для каждого из типов вершин. В частности, для вершин $v_{i} \in V^{S}$ (ЦП) должны быть заданы номер пути $w$, тип стрелочного перевода $\tau_{\text {c }}$ его направление $S$ и, при необходимости, длины прямых вставок $f_{P}, f_{B}$ до вершин, смежных с $v_{i}\left(\mathbf{X}^{S}=\left\{w, \tau_{c}, S, f_{P}, f_{B}\right\}\right)$. 
Таблица 2.3 - Каноническая модель станции А

\begin{tabular}{|c|c|c|c|c|c|c|c|c|}
\hline$N$ & $N_{P}$ & $N_{B}$ & $W$ & $S$ & $T$ & $f_{P}$ & $f_{B}$ & \\
\hline 1 & 9 & 3 & 2 & 1 & 1 & 9999 & & \\
\hline 3 & 5 & 0 & 1 & 1 & 1 & & & \\
\hline 5 & 4 & 7 & 1 & 0 & 0 & 9999 & & \\
\hline 7 & +6 & 0 & 2 & 0 & 0 & 300 & & \\
\hline 9 & +7 & 11 & 2 & 0 & 0 & & & \\
\hline 11 & 201 & 202 & 0 & 0 & 0 & & & \\
\hline 13 & 1 & 0 & 2 & 1 & 0 & & & \\
\hline 2 & 104 & 0 & 2 & 1 & 1 & & & \\
\hline 4 & 105 & +2 & 1 & 0 & 1 & & & \\
\hline 6 & 2 & 0 & 2 & 1 & 0 & & & \\
\hline$N$ & $N_{P}$ & $N_{B}$ & $W$ & $R$ & $L_{P}$ & $\alpha^{\circ}$ & $\alpha^{\prime}$ & $\alpha^{\prime \prime}$ \\
\hline 201 & 203 & 0 & 3 & 300 & & & & \\
\hline 202 & 103 & 0 & 0 & 200 & 58.00 & -4 & -30 & -10 \\
\hline 203 & 6 & 0 & 3 & 400 & & & & \\
\hline 204 & 13 & 0 & 0 & 200 & & & & \\
\hline 205 & 204 & 0 & 4 & 200 & 6.25 & 204 & & \\
\hline$N$ & $N_{P}$ & $N_{B}$ & $W$ & $L_{P}$ & & & & \\
\hline 101 & +13 & 0 & 2 & & & & & \\
\hline 102 & +3 & 0 & 1 & & & & & \\
\hline 103 & 0 & 0 & 0 & & & & & \\
\hline 104 & 0 & 0 & 0 & & & & & \\
\hline 105 & 0 & 0 & 1 & & & & & \\
\hline 106 & 205 & 0 & 4 & 51 & & & & \\
\hline
\end{tabular}


Для каждой вершины $v_{i}$, расположенной на одном из горизонтальных путей, должен быть указан ненулевой номер $w$ этого пути. Принадлежность вершины некоторому горизонтальному пути позволяет в дальнейшем определить ее ординату $\left(Y\left(v_{i}\right)=Y(w)\right)$, используя заданные значения ширины междупутий. Если некоторая вершина $v_{i}$ не принадлежит ни одному из горизонтальных путей, то для нее принимается $w=0$.

Тип стрелочного перевода $\tau_{\mathrm{c}}$ представляет собой номер $\left(\tau_{\mathrm{c}}=0,1, \ldots\right)$, под которым данный стрелочный перевод записан в таблице характеристик. Характеристики стрелочного перевода включают марку крестовины $1 / N$, тип рельса, основные размеры $(a, b)$, угол стрелки $\alpha$ и его тригонометрические функции, а также вставку $k_{0}$.

Направление стрелочного перевода $S$ позволяет различать левосторонние $(S=0)$ и правосторонние $(S=1)$ стрелочные переводы.

Кроме перечисленных параметров, в особых случаях указывают также данные о прямых вставках, укладываемых вправо от данного стрелочного перевода в направлении прямого $\left(f_{\mathrm{p}}\right)$ и/или бокового $\left(f_{\mathrm{B}}\right)$ путей. Как правило, указанные прямые вставки определяются автоматически по схеме взаимного расположения стрелок и в исходных данных не приводятся. Данные о них нужно указывать лишь в следующих случаях:

- при необходимости выбора величины конструктивной вставки, отличающейся от установленной инструкцией для данной схемы взаимного расположения стрелок;

- при необходимости задать длину одного из путей парка для перехода из левой горловины в правую (при расчете координат).

В этих двух случаях задаваемая вставка представляет собой положительное число в пределах $0<f<9999$.

В отдельных случаях (см. рис. 2.4) величина вставки $f$ определяется шириной некоторого междупутья $g$, которую нельзя определить из схемы по разности ординат горизонтальных путей. В этих случаях вместо вставки $f$ не- 
обходимо задать величину требуемого междупутья $g$ со знаком '-'; в противном случае будет принято его стандартное значение $g=5,3$ м.

a)

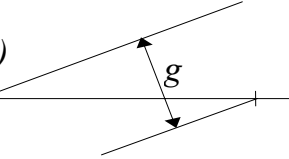

б)

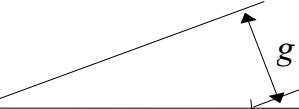

Рисунок 2.4 - Схемы взаимного расположения стрелочных переводов, требующих явного задания ширины междупутья $g$

Если же необходимую ширину междупутья можно определить по разности ординат горизонтальных путей (см. рис. 2.5), то величину $g$, напротив, задавать не следует.

Наконец, в случаях, когда величина вставки должна быть определена по разности координат смежных точек, тогда вместо вставки указывается число 9999. Обычно это имеет место, когда расстояние между смежными стрелочными переводами определяется из условия замкнутости некоторого контура в схеме, а также когда оно зависит от заданной длины одного из путей парка.
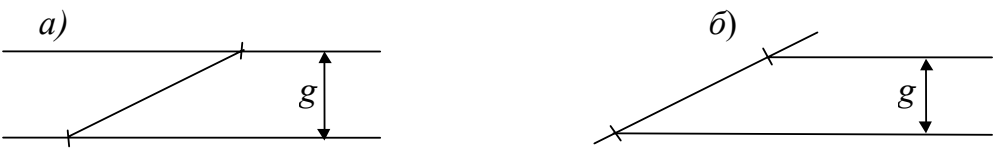

Рисунок 2.5 - Схемы взаимного расположения стрелочных переводов, в которых ширина междупутья $g$ определяется автоматически

Перечисленные возможности обеспечивают необходимую гибкость при проектировании путевого развития станции.

Для вершин $v_{i} \in V^{C}(\mathrm{BУ})$ должны быть заданы номер пути $w$ и радиус кривой $R$, а при необходимости - длина прямой вставки $f_{\mathrm{P}}$ на отрезке до вершины, смежной с $v_{i}$, и угол поворота кривой $\alpha\left(\mathbf{X}^{C}=\{w, R, f, \alpha\}\right)$.

Угол $\alpha$ и/или прямую вставку $f_{\mathrm{P}}$ следует обязательно задавать в тех случаях, когда они не могут быть определены автоматически из соответствующего фрагмента схемы. Обычно это имеет место, когда неизвестны или 
одинаковы ординаты границ отрезка, так что невозможно по их разности определить значения параметров $f_{\mathrm{P}}$ и $\alpha$. Так, в рассматриваемом примере (см. рис. 2.3) для ВУ 202 должны быть заданы значения вставки 202-103 $\left(f_{\mathrm{P}}\right)$ и угла $\alpha$ (см. табл. 2.3).

Отдельно необходимо остановиться на случае, когда кривая является элементом сокращенного конечного соединения путей, так что величина угла поворота не может быть найдена из схемы как сумма образующих его стрелочных углов. В этом случае один из параметров (угол $\alpha$ или вставка $f_{\mathrm{P}}$ ) может быть рассчитан по данным о соединении (см. п. 4.4); при этом другой параметр должен быть задан.

Для расчета неизвестного угла $\alpha$ поворота кривой в сокращенном соединении необходимо определить расчетный путь (см. п. 4.4) и указать в модели для начальной вершины расчетного пути его конечную вершину, а также длину вставки $f_{\mathrm{P}}$ между ними. (см. табл. 2.3, список параметров вершины 205, являющейся начальной вершиной расчетного пути 205-204, по элементам которого определяется неизвестный угол в вершине 204).

При необходимости может быть рассчитана неизвестная вставка $f_{\mathrm{P}}$ при заданном значении угла в сокращенном соединении, которое в этом случае должно быть указано в модели. Так, в рассматриваемом примере можно задать некоторую величину угла (для примера, $3^{\circ} 10^{\prime} 15^{\prime \prime}$ ) в вершине 204 и рассчитать вставку 205-204; ниже приведены соответствующие строки канонической модели (см. табл. 2.3):

\begin{tabular}{|l|l|l|l|l|l|l|l|l|}
\hline $\mathrm{N}$ & $\mathrm{P}$ & $\mathrm{B}$ & $\mathrm{W}$ & $\mathrm{R}$ & $\mathrm{L}_{P}$ & $\alpha^{\circ}$ & $\alpha^{\prime}$ & $\alpha^{\prime \prime}$ \\
\hline 204 & 13 & 0 & 0 & 200 & 0 & 3 & 10 & 15 \\
\hline 205 & 204 & 0 & 4 & 200 & & & & \\
\hline
\end{tabular}

Угол поворота должен быть выражен в градусах, минутах и секундах со знаком (указывают знак плюс, если поворот пути от первоначального направления против часовой стрелки). При этом необходимо придерживаться принятой ориентации дуг графа схемы - слева направо. 
Наконец, для вершин $v_{i} \in V^{W}\left(\right.$ КП) может быть задано расстояние $f_{P}$ до вершины, смежной с $v_{i}$, в случаях, когда соответствующий путь в схеме станции имеет определенную длину (например, тупиковый путь заданной длины). Во всех остальных случаях принимается $f_{\mathrm{P}}=0$; при этом соответствующий путь на плане выравнивается по крайнему левому концу чертежа станции.

\subsection{2. Модель горизонтальных путей}

Горизонтальные пути станции могут быть представлены с помощью древовидного графа $D=(W, H)$, где $W$ - множество вершин, представляющих горизонтальные пути станции (парка, горловины), $H$ - множество ребер, которые соответствуют междупутьям, разделяющим указанные пути [26].

Каждый путь $w$ в графе $D$ характеризуется вектором параметров:

$$
\mathbf{R}=\left(w, Y, C_{\mathrm{n}}, \eta\right)
$$

где $\quad w$ - номер пути;

$Y$ - ордината пути;

$C_{\text {п }}$ - категория пути (главный, приемоотправочный, прочий);

$\eta$ - условия укладки стрелочных переводов (нормальные, стесненные).

Один из узлов дерева, соответствующий пути с заданной ординатой, является его корнем $r$.

Каждому ребру дерева ставится в соответствие ширина междупутья, заданная между путями, указанными в соответствующих узлах. В соответствии с определением дерева число междупутий (ребер) независимо от конфигурации схемы равно $M-1$, где $M$ - число узлов (путей в схеме). Предложенная древовидная структура является удобным средством для определения ординат $Y_{i}$ всех горизонтальных участков путей станции и принадлежащих им точек по заданной ординате $Y_{0}$ опорной точки, находящейся на одном из них, и ширине междупутий. Машинное представление дерева основано на использовании связных списков. При этом, каждый узел дерева содержит вектор параметров $\mathbf{R}$, а также указатель на путь-предок и расстояние $g$ до оси этого пути. Представление дерева с использованием указателей, ведущих от по- 
томков к предкам, выбрано, так как в этом случае в каждом узле необходим только один указатель.

Для расчета ординат горизонтальных путей станции значения $E$ ширины междупутий представляются в виде списка, в котором для каждого из них

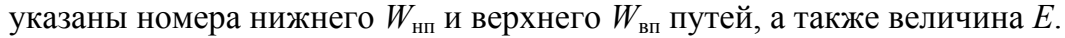

Кроме того, для расчёта координат точек плана станции должна быть задана опорная точка, к которой осуществляется привязка координат всех остальных его точек. В качестве опорной точки выбирается один из ЦП (номер вершины $N_{0}$ ), который находится на горизонтальном пути станции; для данного ЦП должны быть заданы координаты $X_{0}$ и $Y_{0}$.

Указанные данные для станции, схема которой показана на рис. 2.3, приведены в табл. 2.4.

Таблица 2.4 - Внутренняя модель горизонтальных путей станции А

\begin{tabular}{|c|c|c|}
\hline$N_{\mathbf{O}}$ & $X_{\mathbf{O}}$ & $Y_{\mathbf{O}}$ \\
\hline 1 & 200 & 0,33 \\
\hline$W_{\text {нп }}$ & $W_{\text {вп }}$ & $E$, м \\
\hline 1 & 2 & 5,3 \\
\hline 2 & 3 & 9,5 \\
\hline 2 & 4 & 8,2 \\
\hline
\end{tabular}

\subsection{3. Представление сигналов во внутренней модели станщии}

Во внутренней модели сигналы представлены списком $\mathbf{Q}$ каждый элемент которого описывается структурой:

$$
\mathbf{q}_{\mathrm{BH}}=\left(v, z, k_{q}, N_{q}\right),
$$

где $v \in V^{S}$ - номер стрелочного перевода, у которого устанавливается сигнал;

$z$ - направление установки сигнала относительно стрелочного перевода: 0 - за крестовиной на прямом пути; 1 - за крестовиной на боковом пути; 2 - в створе изолирующего стыка рамного рельса.

Номер сигнала $N_{q}$ и его тип $k_{q}$ устанавливают по данным входной модели (2.6). 


\section{3. Внутренняя модель горочной горловины сортировочного парка}

\subsection{1. Каноническая модель спускной части горки}

Модель горочной горловины может быть представлена в виде ориентированного бинарного дерева $G_{\text {г }}=(V, E)$ с корнем $r \in V$. В дереве выделено три подмножества вершин: $V^{S}, V^{C}$ и $V^{W}$. Вершины $v_{i} \in V^{S}$ являются ЦП, $v_{j} \in V^{C}$ - ВУП кривых на спускной части горки, вершины $v_{k} \in V^{W}-$ ВУП кривых на сортировочных путях. Принято, что корнем дерева должна быть головная стрелка горловины $\left(r \in V^{S}\right)$.

Дуга дерева $e=(v, u)$ обозначается упорядоченной парой, состоящей из начальной $v$ и конечной $u$ вершин; направление задано от вершины $v$ к вершине $u$. Принято, что все дуги ориентированы слева направо.

Полустепени исхода вершин $v$ зависят от их типа. Для большинства стрелочных переводов (вершины $\left.v_{i} \in V^{S}\right) d^{+}\left(v_{i}\right)=2$. Исключение составляет головная стрелка (корень дерева $r$ ), для которой $d^{+}(r)=1$, если она пошерстная. Для пошерстных стрелок, которые могут укладываться для обеспечения выхода с крайних путей сортировочного парка в обход горба горки, также $d^{+}\left(v_{i}\right)=1$. Для ВУП на спускной части горки $\left(v_{j} \in V^{C}\right) d^{+}\left(v_{i}\right)=1$, на сортировочных путях $\left(v_{k} \in V^{W}\right)-d^{+}\left(v_{k}\right)=0$. Полустепени захода всех вершин, кроме корня, по определению бинарного дерева равны единице $\left(d^{-}(v)=1\right)$; для корня $d^{-}(r)=0$. Таким образом, для идентификации вершин дерева недостаточно информации об их полустепенях исхода и захода; для разделения множества

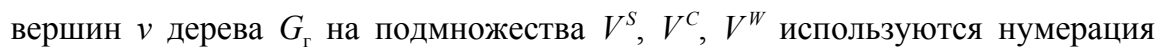
вершин.

Нумерация вершин дерева $G_{\text {г }}$ осуществляется в следующем порядке. Вначале вершины $v_{i} \in V^{S}$ и $v_{j} \in V^{C}$ объединяют в одну общую группу и каждой из них присваивают порядковый номер $\left(v_{i}=1,2, \ldots, 99\right)$. При этом головная стрелка должна быть в списке вершин первой $(r=1)$; остальные вершины могут быть расположены в произвольном порядке. Затем для идентификации 
типа вершин номера ВУП $\left(v_{j} \in V^{C}\right)$ увеличивают на 100 , так чтобы $v_{i}<100$, $v_{j}>100$.

Пути сортировочного парка нумеруют последовательными номерами $m=1,2, \ldots, M$ ( $M$ - число путей в парке), начиная с наиболее удаленного от оси парка. ВУП на сортировочных путях $\left(v_{k} \in V^{W}\right)$ присваивают номера соответствующих путей, увеличенные на $200\left(v_{k}=201,202, \ldots, 200+M\right)$. Пример нумерации вершин горловины приведён на рис. 2.6.

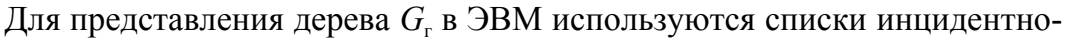
сти. Учитывая, что $G_{\text {г }}$ является ориентированным бинарным деревом, каждой его вершине инцидентны не более двух дуг $\left(v \rightarrow u_{л}\right.$ и $\left.v \rightarrow u_{\text {п }}\right)$. При этом во всех случаях необходимо различать левую $u_{\text {л }}$ п правую $u_{\text {п }}$ вершины, смежные с вершиной $v$. Ориентация осуществляется по направлению угла поворота в вершине $v$ (углу поворота против часовой стрелки соответствует дуга $v \rightarrow u_{\text {Л }}$ ).

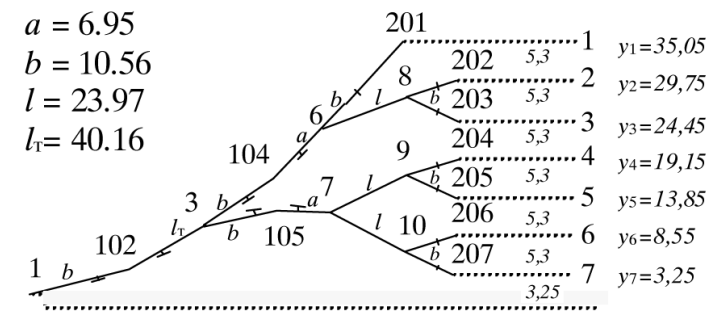

Рисунок 2.6 - Схема горочной горловины

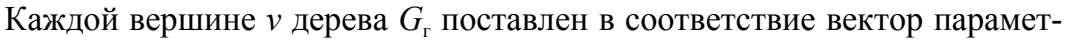
ров $\left(\mathbf{X}_{i}^{S}, \mathbf{X}_{j}^{C}, \mathbf{X}_{k}^{W}\right)$, компоненты которого определяются типом вершины:

a) стрелочный перевод $\left(v_{i} \in V^{S}\right)$ :

$$
\mathbf{X}^{S}=\left(l_{\pi}, l_{\pi}\right),
$$

где $l_{\text {л }}, l_{\text {п }}$ длина прямолинейной части соответственно дуг $v \rightarrow u_{\text {л }}$ и $v \rightarrow u_{\text {п }}$.

б) ВУП на спускной части $\left(v_{j} \in V^{C}\right)$ :

$$
\mathbf{X}^{C}=(\alpha, l, R, \psi),
$$

где $\alpha$ - абсолютная величина угла поворота; если $\alpha=0$, это является признаком необходимости расчета угла программой; 
$l$ - длина прямолинейной части дуги $(v \rightarrow u)$;

$R$ - радиус кривой;

$\psi$ - признак включения длины кривой в длину отрезка $l$ (при $\psi=1$ заданная длина отрезка $l$ уменьшается на длину кривой $l_{c}\left(l^{\prime}=l-l_{c}\right)$; при $\psi=0$ заданная длина отрезка $l$ сохраняется независимо от длины кривой $l_{c}$ ).

в) ВУП на сортировочном пути $\left(v_{k} \in V^{W}\right)$ :

$$
\mathbf{X}^{W}=(Y, R)
$$

где $\quad Y$ - ордината пути относительно оси сортировочного парка;

$R$ - радиус кривой.

Внутренняя модель горочной горловины, изображенной на рис. 2.6, приведена в табл. 2.5.

Таблица 2.5 - Внутренняя модель горочной горловины

\begin{tabular}{|c|c|c|c|c|c|c|c|c|c|}
\hline$v$ & $u_{\text {л }}$ & $U_{\text {п }}$ & $\alpha^{\circ}$ & $\alpha^{\prime}$ & $\alpha^{\prime \prime}$ & $l_{\text {л }}$ & $L_{\text {п }}$ & $R$ & $\psi$ \\
\hline 1 & 102 & 0 & 0 & 0 & 0 & 10.56 & 0 & 0 & 0 \\
\hline 102 & 3 & 0 & 0 & 0 & 0 & 40.16 & 0 & 200 & 0 \\
\hline 3 & 104 & 105 & 0 & 0 & 0 & 10.56 & 10.56 & 0 & 0 \\
\hline 104 & 6 & 0 & 1 & 50 & 52 & 6.95 & 0 & 200 & 0 \\
\hline 105 & 0 & 7 & 1 & 50 & 52 & 0 & 6.95 & 200 & 0 \\
\hline 6 & 201 & 8 & 0 & 0 & 0 & 10.56 & 23.97 & 0 & 0 \\
\hline 7 & 9 & 10 & 0 & 0 & 0 & 23.97 & 23.97 & 0 & 0 \\
\hline 8 & 202 & 203 & 0 & 0 & 0 & 10.56 & 10.56 & 0 & 0 \\
\hline 9 & 204 & 205 & 0 & 0 & 0 & 10.56 & 10.56 & 0 & 0 \\
\hline 10 & 206 & 207 & 0 & 0 & 0 & 10.56 & 10.56 & 0 & 0 \\
\hline
\end{tabular}

Необходимо заметить, что заданные на первом этапе вершины $V^{W}$ являются условными и используются для описания топологии проектируемой схемы. В действительности в конечных соединениях на некоторых путях парка на следующем этапе проектирования приходится укладывать дополнительные кривые (ВУП $\left.V^{\mathrm{B}}\right)$. ВУП указанных кривых $\left(v_{k}^{\prime} \in V^{\mathrm{B}}\right)$ делят соответствующие дуги $\left(v_{i}, v_{k}\right), v_{i} \in V^{S}, v_{k} \in V^{W}$ на две новые дуги: $\left(v_{i}, v_{k}^{\prime}\right)$ и $\left(v_{k}^{\prime}, v_{k}\right)$. Дополнительным вершинам $v_{k}^{\prime}$ присваиваются номера основных вершин, увеличенные на $100\left(v_{k}^{\prime}=301,302, \ldots, 300+M\right)$. Обычно указанные вершины и 
связанные с ними параметры появляются уже в процессе проектирования и в исходное описание конструкции горловины не включаются. Однако, в некоторых случаях, например, на промежуточных этапах проектирования, дополнительные вершины включают в описание схемы. В этих случаях вектор $\mathbf{X}^{W}$ включает компоненты:

$$
\mathbf{X}^{W}=\left(Y, R, \alpha_{\text {д }}, R_{\text {д }}, K\right)
$$

где $\quad \alpha_{\text {д }}-$ угол поворота дополнительной кривой с учетом знака (знак плюс при повороте против часовой стрелки);

$R_{\text {д }}$ - радиус дополнительной кривой;

$K_{\text {д }}$ - длина дополнительной прямой вставки, укладываемой между крестовиной последнего стрелочного перевода и началом дополнительной кривой; при отсутствии вставки принимается $K_{д}=0$.

Следует заметить, что длина вставки между основной и дополнительной кривыми не задается, т.к. она может быть рассчитана по остальным известным данным.

\subsection{2. Модель сортировочных путей}

Каждой вершине угла поворота на сортировочном пути ставится в соответствие вектор параметров $\mathbf{X}_{W}$ :

$$
\mathbf{X}_{W}=(Y, R)
$$

где $\quad Y$-ордината пути относительно оси сортировочного парка;

$R$ - радиус кривой.

Следует заметить, что радиусы кривых на расчетных путях с трудными условиями вписывания обычно принимают равными минимальному значению $180 \mathrm{M}$.

Список указанных параметров $\mathbf{X}_{W}$ для горочной горловины, схема которой показана на рис. 2.6, приведен в табл. 2.6.

Для привязки горочной горловины к существующей системе координат должны быть заданы координаты головной стрелки в этой системе $X_{1}, Y_{1}$. В практике проектирования горочных горловин приняты следующие соглаше- 
ния [20]. Горочная горловина делится осью сортировочного парка на две части - левую и правую, проектирование которых выполняется, как правило, независимо. Проектирование ведется в прямоугольной системе координат в первой четверти; если горочная горловина расположена по другому, то она предварительно соответствующим образом разворачивается. За ось абсцисс $O X$ принята ось сортировочного парка; ось ординат проходит через головную стрелку горловины (см. рис. 2.6).

Таблица 2.6 - Модель сортировочных путей горочной горловины

\begin{tabular}{|r|r|l|}
\hline BY & \multicolumn{1}{|c|}{ Y } & R \\
\hline 201 & 35,05 & 180 \\
202 & 29,75 & 200 \\
203 & 24,45 & 200 \\
204 & 19,15 & 200 \\
205 & 13,85 & 200 \\
206 & 8,55 & 200 \\
207 & 3,25 & 200 \\
\hline
\end{tabular}

При принятой древовидной структуре представления горловины при наличии в ее конструкции перекрестного съезда в качестве начального принимается обычно выходной (пошерстный) стрелочный перевод этого съезда (корень дерева). В этой связи для сохранения общепринятого начала координат предусмотрена возможность задания координат $\left(x_{1}, y_{1}\right)$ начальной стрелки в системе $O X У$.

Для расчетов углов наклона к оси $O X$ всех элементов горловины должен быть задан угол наклона $\varphi$ базисного элемента горловины к оси $O X$; в качестве базисного элемента принята ось симметрии начального стрелочного перевода.

Для определения положения парковых тормозных позиций необходимо указать минимальную ширину междупутья $e_{\min }$, при которой можно устанавливать замедлители на парковых путях. 


\section{4. Выходные модели плана путевого развития станций}

Выходная модель путевого развития станций должна содержать совокупность параметров, необходимых для решения поставленной задачи. Разработанная система геометрических моделей может быть использована для решения достаточно широкого круга задач - построения масштабных планов и схем станций, подготовки технической документации, организации интерактивного синтеза планов путевого развития станций сложной конструкции, создания функциональных моделей для имитационного моделирования и др. Возможная структура выходных моделей для решения некоторых задач и перечень содержащихся в них данных приведены ниже.

\subsection{1. Расчетные параметры плана путевого развития}

Универсальной формой выходной модели является совокупность расчетных параметров плана путевого развития станций, которая включает данные о сопрягающих кривых, расстояниях между характерными точками плана и их координатах.

Информация о параметрах сопрягающих кривых представляется структурами:

$$
\mathbf{C}_{i}=\{v, \alpha, R, T, K\}, i=1,2, \ldots, n_{C},
$$

где $\quad v$ - номер вершины $v \in V C ; \alpha-$ угол поворота кривой в градусах, минутах, секундах; $R$ - радиус; $T$ - тангенс; $K$ - длина кривой; $n_{C}-$ число кривых на плане.

Данные о расстояниях между характерными точками плана (центрами стрелочных переводов, вершинами углов поворота, концами путей) представляются структурами:

$$
\mathbf{E}_{j}=\{v, u, l, f\}, j=1,2, \ldots, n_{E},
$$

где $v, u$ - номера, соответственно, левой и правой точек отрезка; $l-$ общее расстояние между точками; $f$ - длина прямой вставки; $n_{E}-$ число отрезков плана. 
Координаты характерных точек плана представляются структурами:

$$
\mathbf{P}_{k}=\{v, x, y\}, k=1,2, \ldots, n_{P},
$$

где $v$ - номер вершины; $x, y$ - координаты точки в локальной системе; $n_{P}$ - число точек на плане.

Для расчета показателей проекта по данным выходной модели определяются строительная $L_{\text {стр }}$ и полная $L_{\text {полн }}$ длины путей

$$
\begin{gathered}
L_{\text {стр }}=\sum_{i=1}^{n_{c}} K_{i}+\sum_{j=1}^{n_{E}} f_{j} \\
L_{\text {полн }}=L_{\text {стр }}+2 \cdot \sum_{q=1}^{n_{S}} L_{\text {оq }}
\end{gathered}
$$

где $L_{\mathrm{o}}-$ полная длина стрелочного перевода.

Выходная модель горочной горловины содержит списки параметров сопрягающих кривых на спускной части горки и на сортировочных путях, данные о расстояниях между точками и координатах центров стрелочных переводов. Кроме того, для горочных горловин выходная модель дополнительно включает координаты точек, определяющих места возможной установки замедлителей на сортировочных путях, а также суммы углов поворота на маршрутах скатывания отцепов.

Данные о возможном расположении парковых замедлителей представляются структурами:

$$
\mathbf{R}_{g}=\{g, g+1, x, y\}, g=1,2, \ldots, n_{W^{-}} 1,
$$

где $g$ - номер сортировочного пути; $x, y$ - координаты точки, находящейся между путями $(g, g+1)$ и удаленной от них на расстояние $e_{\min } / 2$, при котором допускается установка парковых замедлителей; $n_{W}$ - число сортировочных путей в парке.

Информация об алгебраических суммах углов по маршрутам скатывания отцепов с горки, необходимая для выбора расчетного трудного пути при определении высоты горки, представляется списком структур:

$$
\mathbf{A}_{g}=\left\{g, \Sigma \alpha_{\mathrm{c}}, \Sigma \alpha_{\Pi}, \Sigma \alpha\right\}, \quad g=1,2, \ldots, n_{W},
$$


где $\Sigma \alpha_{c}, \Sigma \alpha_{n}, \Sigma \alpha-$ алгебраические суммы углов поворота на маршруте скатывания отцепов на путь $g$, соответственно, на спускной части горки, на сортировочном пути и общая.

Результаты расчета параметров плана путевого развития станции, схема которой показана на рис. 2.3, приведены в Приложении А (табл. А.2).

Результаты расчета параметров плана горочной горловины, схема которой показана на рис. 2.6, приведены в Приложении Б.

\subsection{2. Модель для интерактивного проектирования крупных станциий}

Разработанная система геометрических моделей обеспечивает расчет плана путевого развития отдельных горловин либо относительно несложных станций с прямолинейными горизонтальными путями. Крупные станции, состоящие из нескольких парков, а также станции, которые целиком или частично располагаются на кривых, необходимо разбить на базовые элементы (блоки), в пределах которых пути остаются прямолинейными и горизонтальными. Для каждого блока выполняются предварительные расчеты, результаты которых представляются в виде выходной модели определенной структуры. В дальнейшем выходные модели блоков используются для их объединения в общем плане станции, которое осуществляется в интерактивном режиме. Указанный режим обеспечивает возможность выбора и оперативного изменения положения каждого блока на плане станции. При этом всякое объединение блоков можно снова рассматривать как отдельный блок, используя его для продолжения процесса автоматизированного проектирования крупных станций.

Таким образом, план путевого развития крупной станции может быть представлен совокупностью блоков $\mathbf{B}_{1}, \mathbf{B}_{2}, \ldots, \mathbf{B}_{n}, n-$ число блоков. Каждый блок представляется выходной моделью $\mathbf{B}_{i}$, содержащей данные о его элементах:

$$
\mathbf{B}=\left\{G, v_{\mathrm{o}}\right\},
$$

где $G$-ориентированный граф схемы путевого развития блока;

$v_{\mathrm{o}}$ - базовая вершина (опорная точка). 
Ориентированный граф $G=(V, E)$ представляется множеством вершин $v_{i} \in V$, каждая из которых характеризуется вектором:

$$
v=\left\{N, N_{\mathrm{P}}, N_{\mathrm{B}}, x, y, \mathbf{Z}\right\},
$$

где $\quad N$-номер вершины;

$N_{\mathrm{P}}$ - номер вершины по прямому пути;

$N_{\mathrm{B}}$ - номер вершины по боковому пути;

$x, y$ - координаты вершины в локальной системе координат блока;

$\mathbf{Z}$ - список конструктивных параметров.

Значения параметров списка $\mathbf{Z}$ зависят от типа вершины: для ЦП данный список содержит тип стрелочного перевода, для ВУП - значение радиуса кривой, для КП - тип окончания (0 - конец пути, 1 - упор).

Для размещения блока на общем плане ему необходимо присвоить но-

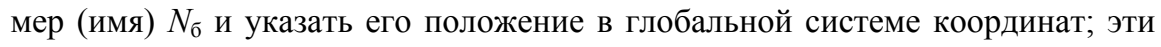
данные для каждого блока представляются структурой

$$
\mathbf{P}=\left\{N_{\tilde{\sigma}}, \varphi, X, Y\right\},
$$

где $N_{\text {б }}$ - номер (имя) блока;

$\varphi$ - угол поворота блока относительно начала координат глобальной системы;

$X, Y$ - координаты точки размещения начала координат блока в глобальной системе координат.

Параметры $(\varphi, X, Y)$ являются переменными величинами и могут интерактивно изменяться в процессе проектирования.

В процессе построения плана станции определенные пути пары объединяемых блоков $\mathbf{B}_{i}, \mathbf{B}_{j}$ сопрягаются круговыми кривыми. Для реализации указанной процедуры для сопрягаемых путей должен быть задан список структур:

$$
\mathbf{U}_{k}=\left\{N_{i}, v_{i k}, N_{j}, v_{j k}, R_{k}\right\}, k=1,2, \ldots, m
$$

где $N_{i}, N_{j}$ - номера объединяемых блоков;

$v_{i k}, v_{j k}$ - номера вершин КП $\left(v_{k} \in V^{W}\right)$, ограничивающих сопрягаемые пути в блоках; 
$R_{k}$ - радиус сопрягающей кривой;

$m$ - число сопрягаемых пар путей.

\subsection{3. Модель для построения масштабных планов станций}

Выходная модель станции может быть представлена в виде графического файла. В качестве формата выходного файла принят формат обмена графическими данными AutoCAD dxf, который поддерживается большинством графических редакторов. Данный файл представляет собой текстовое описание графического изображения в виде ассоциативного списка. В файле dxf выделены 7 секций: HEADER, CLASSES, TABLES, BLOCKS, ENTITIES, OBJECTS, THUMBNAILIMAGE. В качестве основы для формирования файла используется специальный шаблон, в секции BLOCKS которого описаны графические изображения тангенсов, сигналов и стрелочных переводов на планах станций (см. рис. 2.7).

Формирование чертежа заключается в заполнении секции ENTITIES. При этом прямолинейные участки путей и участки кривых представляются в виде полилиний AutoCAD, каждая из которых имеет две вершины. Описание полилиний представлено в табл. 2.7.

Прямолинейные участки путей создаются на основании дуг графа $e \in E$. В случае, если вершиной дуги является центр стрелочного перевода, либо конец пути, то соответствующей вершине полилинии присваиваются координаты вершины дуги. Если же вершиной дуги является вершина угла поворота круговой кривой, то координаты вершины полилинии определяются по формулам:

$$
x=x_{\mathrm{H}}+\frac{x_{e \kappa}-x_{e \mathrm{H}}}{l_{\mathrm{e}}} k, y=y_{\mathrm{H}}+\frac{y_{e \kappa}-y_{e \mathrm{e}}}{l_{\mathrm{e}}} k,
$$

где $k=T$ для начальной вершины и $k=l_{\mathrm{e}}-T$ для конечной вершины. 
a)

b)

1/11 правосторонний

1/11 левосторонний б)

1/9 правосторонний

1/9 левосторонний

2)

Рисунок 2.7 - Блоки, используемые при построении плана станции: $a$ знак тангенса; $\sigma$ - нецентрализованный стрелочный перевод; 6 - централизованные стрелочные переводы; 2 - изображения сигналов

Участки круговых кривых создаются на основании вершин графа $v \in V^{C}$. При этом координаты начальной точки кривой определяются с помощью выражения (2.11) по данным заходящей дуги при $k=l_{\mathrm{e}}-T$, а координаты конечной точки - по данным исходящей дуги при $k=T$. Коэффициент кривизны определяется с помощью выражения

$$
\varphi=\operatorname{tg}\left(\alpha_{\mathrm{\kappa}} / 4\right),
$$

где $\alpha_{\kappa}-$ угол поворота кривой.

Таблица 2.7 - Описание полилиний в файле $\mathrm{dxf}$

\begin{tabular}{|c|l|}
\hline $\begin{array}{c}\text { Идентификатор } \\
\text { поля }\end{array}$ & \multicolumn{1}{c|}{ Описание поля } \\
\hline 0 & Тип объекта (LWPOLYLINE) \\
\hline 100 & Имя подкласса (AcDbPolyline) \\
\hline 90 & Число вершин \\
\hline 70 & Флаг замкнутости \\
\hline 43 & Ширина полилинии \\
\hline 10 & Абсцисса вершины (указывается для каждой вершины) \\
\hline 20 & Ордината вершины (указывается для каждой вершины) \\
\hline 42 & Коэффициент кривизны $\varphi$ \\
\hline
\end{tabular}


Условные обозначения тангенсов кривых, стрелочных переводов и сигналов добавляются в чертеж плана станции в виде блоков (см. рис. 2.7). В табл. 2.8 представлено описание этих блоков.

Величины углов поворота блоков, соответствующих стрелочным переводам, определяются на основании углов наклона дуг, соответствующих рамным рельсам, а их имя - на основании типа стрелочного перевода $\tau_{\mathrm{c}}$ и его направления $S$. Величины углов поворота блоков тангенсов устанавливаются в соответствии с углами наклона отрезков, инцидентных вершинам углов поворота.

Таблица 2.8- Описание блоков в файле $\mathrm{dxf}$

\begin{tabular}{|c|l|}
\hline $\begin{array}{c}\text { Идентификатор } \\
\text { поля }\end{array}$ & \multicolumn{1}{c|}{ Описание поля } \\
\hline 0 & Тип объекта (INSERT) \\
\hline 100 & Имя подкласса (AcDbBlockReference) \\
\hline 2 & Имя блока \\
\hline 10 & Абсцисса точки вставки блока \\
\hline 20 & Ордината точки вставки блока \\
\hline 50 & Угол поворота блока, $\alpha_{\text {Б }}$ \\
\hline
\end{tabular}




\section{ГЛАВА 3 \\ АВТОМАТИЗИРОВАННОЕ ФОРМИРОВАНИЕ СИСТЕМЫ ГЕОМЕТРИЧЕКИХ МОДЕЛЕЙ ПЛАНА СТАНЦИИ}

\section{1. Ввод немасштабной схемы станции и построение входной модели}

Формирование схемы путевого развития осуществляется путем добавления, удаления и изменения отрезков, соответствующих участкам пути в процессе графического ввода схемы станции. Такой подход позволяет существенно ускорить процесс ввода схемы в ЭВМ по сравнению с ее формированием из отдельных примитивов (стрелок кривых и т.д.) [27]. Для обеспечения совмещения отрезков в центрах стрелочных переводов и вершинах углов поворота реализуется объектная привязка. На первом этапе осуществляется проверка идентичности координат вводимых точек $(x, y)$ конечным точкам $\mathbf{p}$ существующих отрезков. Точки считаются идентичными в случае, когда расстояние между ними не превышает заданной величины погрешности $\varepsilon$ :

$$
\sqrt{(x(\mathbf{p})-x)^{2}-(y(\mathbf{p})-y)^{2}}<\varepsilon,
$$

где $\quad x(\mathbf{p}), y(\mathbf{p})-$ координаты существующих точек.

Далее осуществляется контроль принадлежности вводимых точек существующим отрезкам. При этом точка $A$ считается принадлежащей отрезку $B C$ в случае, если выполняются условия:

$$
\left\{\begin{array}{l}
\left|l_{A}^{B C}\right|<\varepsilon \\
l_{A B}<l_{B C} \\
l_{A C}<l_{B C}
\end{array}\right.
$$

где $\quad l_{A}^{B C}-$ расстояние (со знаком) от точки до прямой;

$l_{A B}, l_{A C}$ - расстояние от точки $A$ до конечных точек отрезка;

$l_{B C}$ - длина отрезка $B C$.

Расчетная схема определения принадлежности точки отрезку приведена на рис. 3.1. 


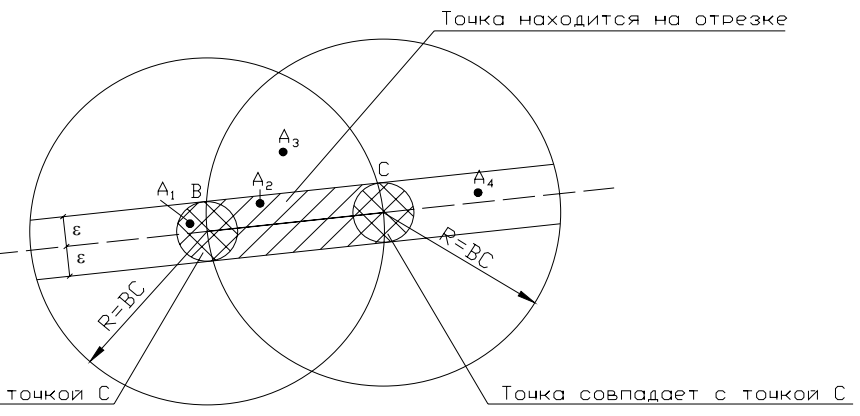

Рисунок 3.1 - Расчетная схема определения принадлежности точки отрезку

Расстояние от точки $A$ до отрезка $B C$ в соответствии с рис. 3.2 может быть определено из выражения

$$
l_{A}^{B C}=A B \cdot \sin \angle A B C=A B \cdot \sin (\alpha-\beta)
$$

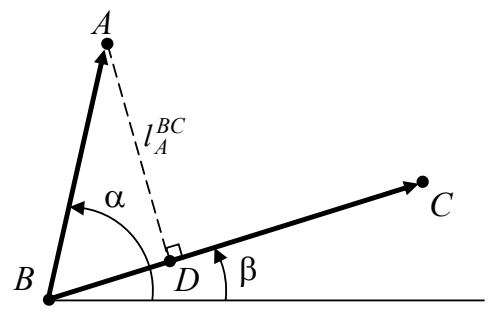

Рисунок 3.2 - Расчетная схема определения координат ближайшей точки отрезка

Значение синуса угла $A B C$ может быть установлено на основании координат точек:

$$
\begin{gathered}
\sin (\alpha-\beta)=\sin \alpha \cos \beta-\sin \beta \cos \alpha, \\
\sin \alpha=\frac{y_{A}-y_{B}}{B A}, \quad \cos \alpha=\frac{x_{A}-x_{B}}{B A}, \sin \beta=\frac{y_{C}-y_{B}}{B C}, \cos \beta=\frac{x_{C}-x_{B}}{B C} .
\end{gathered}
$$

Подставляя величины в выражение (3.2) получаем:

$$
l_{A}^{B C}=\frac{\left(y_{A}-y_{B}\right)\left(x_{C}-x_{B}\right)-\left(y_{C}-y_{B}\right)\left(x_{A}-x_{B}\right)}{\sqrt{\left(x_{C}-x_{B}\right)^{2}+\left(y_{C}-y_{B}\right)^{2}}}
$$

Величина $l_{A}^{B C}$ принимает отрицательное значение если точка лежит 
справа от отрезка, положительное значение, если точка лежит слева от отрезка и 0, если точка и отрезок принадлежат одной прямой.

В случае, если условие (3.1) выполняется, точка $A$ переносится в точку $D$ на отрезке $B C$ (см. рис. 3.2):

$$
x_{D}=\frac{B A}{B C}\left(x_{C}-x_{B}\right) \cos \angle A B C+x_{B}, y_{D}=\frac{B A}{B C}\left(y_{C}-y_{B}\right) \cos \angle A B C+y_{B},
$$

где

$$
\cos \angle A B C=\frac{\overline{B A} \cdot \overline{B C}}{B A \cdot B C} .
$$

Ввиду малости угла $A B C, \cos \angle A B C \approx 1$, для определения координат точки $D$ могут быть использованы упрощенные выражения:

$$
x_{D}=\frac{B A}{B C}\left(x_{C}-x_{B}\right)+x_{B}, y_{D}=\frac{B A}{B C}\left(y_{C}-y_{B}\right)+y_{B} .
$$

Учитывая, что вершины вводимых отрезков должны принадлежать множеству вершин графа $G$ путевого развития станции определяется их степень и, в необходимых случаях, осуществляется модификация. Примеры различных случаев модификации вершин модели при добавлении отрезков приведены в табл. 3.1, варианты 1-5. При этом, в случае, когда новый отрезок примыкает к конечной точке существующего, контролируется величина угла между существующим отрезком $A B$ и новым отрезком $B C$. Для упрощения мерой величины угла отрезка может быть косинус, который, ввиду принятой ориентации отрезков, может иметь только положительные значения.

$$
\cos \angle A B C<1-\delta
$$

где $\delta$ - величина погрешности.

Если данное условие выполняется, то в точку $B$ помещается объект CURVE (см. табл. 3.1, вариант 2); в противном случае конечная точка отрезка $B$ перемещается в точку $C$ (см. табл. 3.1, вариант 3$)$.

При удалении отрезка из модели также определяются степени соответствующих вершин, и в соответствии с ними корректируется модель (см. табл. 3.1, вариант 6-9). 
Таблица 3.1 - Модификация входной модели станции в процессе ввода ее графического изображения

\begin{tabular}{|c|c|c|c|c|}
\hline & $\begin{array}{l}\text { Номер } \\
\text { варианта }\end{array}$ & $\begin{array}{l}\text { До модифика- } \\
\text { ции }\end{array}$ & $\begin{array}{l}\text { После модифика- } \\
\text { ции }\end{array}$ & Действие \\
\hline \multirow{5}{*}{ 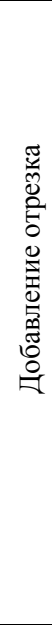 } & 1 & & & - \\
\hline & 2 & & $\overrightarrow{\mathrm{C}}$ & $\begin{array}{l}\text { Угол между отрезками } \\
\text { больше минимального: } \\
\text { поместить в конечную } \\
\text { точку объект CURVE }\end{array}$ \\
\hline & 3 & $\ldots . . .+\dot{C}$ & $\vec{B}$ & $\begin{array}{l}\text { Угол между отрезками } \\
\text { меньше минимального: } \\
\text { объединить отрезки }\end{array}$ \\
\hline & 4 & & & $\begin{array}{l}\text { Удалить в конечной точ- } \\
\text { ке отрезка объект } \\
\text { CURVE и поместить в } \\
\text { нее объект SWITCH }\end{array}$ \\
\hline & 5 & & & $\begin{array}{l}\text { Разбить отрезок на два } \\
\text { отрезка и поместить в } \\
\text { точку объект SWITCH }\end{array}$ \\
\hline \multirow{4}{*}{ 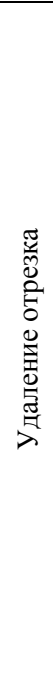 } & 6 & & & - \\
\hline & 7 & & & $\begin{array}{l}\text { Удалить объект CURVE } \\
\text { в конечной точке отрезка }\end{array}$ \\
\hline & 8 & & $\vec{C}$ & $\begin{array}{l}\text { Угол между оставшими- } \\
\text { ся отрезками больше } \\
\text { минимального: удалить } \\
\text { объект SWITCH в ко- } \\
\text { нечной точке отрезка и } \\
\text { поместить в нее объект } \\
\text { CURVE } \\
\end{array}$ \\
\hline & 9 & $A^{*}$ & $\vec{C}$ & $\begin{array}{l}\text { Угол между оставшими- } \\
\text { ся отрезками меньше } \\
\text { минимального: удалить } \\
\text { объект SWITCH в ко- } \\
\text { нечной точке отрезка и } \\
\text { объединить оставшиеся } \\
\text { отрезки }\end{array}$ \\
\hline
\end{tabular}

При этом в случае удаления отрезков, примыкающих к вершине SWITCH, контролируется величина угла наклона между оставшимися отрезками $A B$ и $B C$. В случае, когда условие (3.4) выполняется в точку $B$, добавляется объект CURVE (см. табл. 3.1, вариант 8); иначе оставшиеся отрезки $A B$ и $B C$ объединяются в один $A C$ (см. табл. 3.1, вариант 9). 
При вводе номеров путей указываются координаты точки вставки номера пути $\mathbf{p}_{w}$ и определяется ее принадлежность существующим горизонтальным отрезкам $\mathbf{e}_{i}$ с помощью условий:

$$
\left\{\begin{array}{l}
y\left(\mathbf{p}_{e n, i}\right)=y\left(\mathbf{p}_{e r, i}\right) \\
x\left(\mathbf{p}_{e n, i}\right)<x\left(\mathbf{p}_{w}\right)<x\left(\mathbf{p}_{e \kappa, i}\right) . \\
\left|y\left(\mathbf{p}_{w}\right)-y\left(\mathbf{p}_{e n, i}\right)\right|<\varepsilon
\end{array}\right.
$$

В случае, если указанная точка не принадлежит ни одному из горизонтальных отрезков, выдается сообщение об ошибке.

При вводе междупутий $\mathbf{m}$ проверяется наличие горизонтальных отрезков над и под точкой вставки $\mathbf{p}_{m}$ указателя междупутья. В случае отсутствия хотя бы одного из таких отрезков, выдается сообщение об ошибке.

На рис. 3.3 приведен результат графического ввода схемы станции А, изображенной на рис 2.1 .

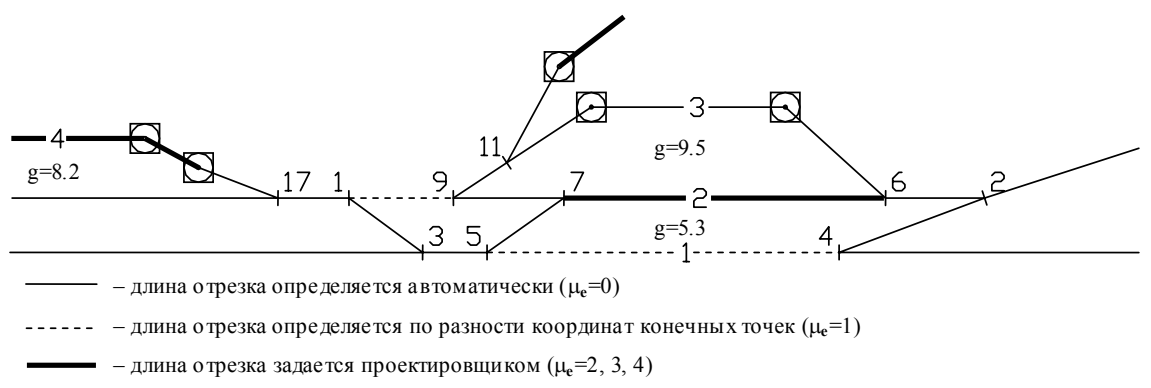

Рисунок 3.3 - Графический ввод немасштабной схемы станции А

\section{2. Формирование модели горизонтальных путей станции}

Построение модели горизонтальных путей станции осуществляется в автоматизированном режиме на основе преобразований входной модели $\Omega_{\mathrm{Bx}}$ (см. п. 2.1). Для этого выполняется поиск горизонтальных участков путей $\mathbf{e}$ во введенной схеме, определение тех пар путей, для которых должна быть задана ширина междупутья, идентификация номеров путей $\mathbf{w}$ и междупутий m, заданных на немасштабной схеме, интерактивный ввод недостающих зна- 
чений ширины междупутий, определение принадлежности вершин орграфа $G$ горизонтальным участкам путей.

Предварительно введем определение горизонтального участка пути орграфа $G$. Простой орцепью орграфа является последовательность дуг $e_{i}$, в которой конечная вершина всякой дуги, отличной от последней, является начальной вершиной следующей, причем все входящие в нее вершины различны. Поскольку в орграфе $G$ нет параллельных дуг, орцепь может быть задана последовательностью входящих в нее вершин: $w=\left(v_{0}, v_{1}, \ldots, v_{n}\right)$. Тогда горизонтальным участком пути является такая простая орцепь, у которой ординаты всех ее вершин одинаковы: $y_{0}=y_{1}=\ldots=y_{n}$. При этом каждый такой путь должен иметь максимальную длину на графе $G$, и значит вершина $v_{0}$ не должна иметь заходящих, а $v_{n}$ - исходящих горизонтальных дуг (т.е. дуг с одинаковыми ординатами концевых вершин).

Поиск горизонтальных путей начинается с сортировки списка дуг орграфа $G$ по возрастанию абсцисс их левых вершин $x(v)$. Далее отсортированные горизонтальные дуги е, включаются в некоторый путь $w_{j}$ при условии совпадения ординат $y\left(v_{1}\right)=y\left(v_{2}\right)=y\left(w_{j}\right)$. После построения всех горизонтальных путей их нумеруют последовательными условными номерами $w_{j}=j$, $j=1, \ldots, M$, где $M$ - число горизонтальных путей в схеме. Для каждого полученного участка пути осуществляется поиск соответствующего объекта $\mathbf{w} \in \Omega_{\text {вх }}$ в соответствии с условием:

$$
\left\{\begin{array}{l}
x\left(w_{0}\right)<x(\mathbf{w})<x\left(w_{n}\right) \\
y\left(w_{0}\right)=y(\mathbf{w})=y\left(w_{n}\right)
\end{array}\right.
$$

В случае отсутствия номера пути $\mathbf{w}$ в списке $\Omega_{\text {вх }}$ пути присваивается условный номер $N_{w}$. Далее всем отрезкам е, включенным в состав горизонтальных путей, ставится в соответствие номер соответствующего пути $N_{w}$.

Для автоматического определения тех пар путей $\left(w_{i}, w_{j}\right), i \neq j$, для которых должна быть задана ширина междупутий, необходимо построить дерево 
$D=(W, H)$ смежных путей станции (см. п. 2.2.2). Для построения дерева $D$ необходимо предварительно построить взвешенный неориентированный граф расстояний между путями $\bar{\Gamma}=(W, \bar{\Lambda})$, каждая вершина которого $w_{i}$ соответствует горизонтальному пути станции. Вес $\Delta y_{i j}$ peбра $\left(w_{i}, w_{j}\right)$ равен абсолютной величине разности экранных ординат соответствующих путей $\left(\Delta y_{i j}=\left|y\left(w_{i}\right)-y\left(w_{j}\right)\right|\right)$. При этом принято, что указанное ребро существует, если существует перпендикуляр, пересекающий оба горизонтальных пути $w_{i}$ и $w_{j}$; в противном случае $\Delta y_{i j}=0$. Формально условие существования ребра $\left(w_{i}, w_{j}\right)$ в графе $\bar{\Gamma}$ можно записать как

$$
\left.\begin{array}{l}
x\left(v_{0 i}\right)<x\left(v_{n j}\right) \\
x\left(v_{0 j}\right)<x\left(v_{n i}\right)
\end{array}\right\}
$$

где $x\left(v_{0}\right), x\left(v_{n}\right)$ - экранные абсциссы, соответственно, начала и конца пути.

На рис. 3.4, а показан граф $\bar{\Gamma}$ для схемы станции, приведенной на рис. 3.3; он представляется квадратной матрицей смежности $\mathrm{A}_{\Gamma}=\left[\Delta y_{i j}\right], i=1, \ldots$, $M, j=1, \ldots, M$. Ниже приведена матрица смежности для данного графа; элементы матрицы $\Delta y_{i j}$ вычислены при значениях междупутий, указанных на рис. 3.3.

$$
\mathrm{A}_{\Gamma}=\left[\begin{array}{rrrr}
- & 9 & 17 & 16 \\
9 & - & 8 & 7 \\
17 & 8 & - & - \\
16 & 7 & - & -
\end{array}\right]
$$

На графе $\bar{\Gamma}$ строится кратчайший остов $\bar{D}$, у которого сумма весов ребер наименьшая. В этом случае в остов $\bar{D}$ будут входить ребра, связывающие только смежные пути станции. Начальные и конечные вершины указанных ребер соответствуют тем парам путей, для которых должна быть задана ширина междупутий. 

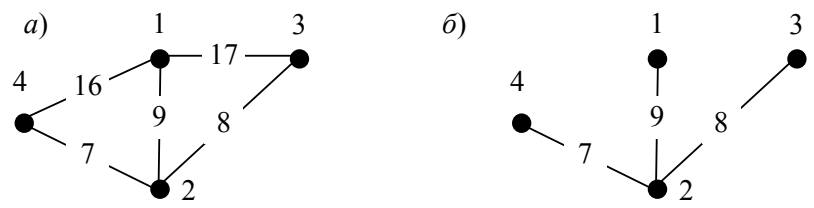

Рисунок 3.4 - Модель горизонтальных путей станции: $a$-граф $\bar{\Gamma}$ горизонтальных путей станции; $\sigma$ - кратчайший остов $\bar{D}$ графа $\bar{\Gamma}$

Для построения кратчайшего остова используется алгоритм Прима [24]; кратчайший остов для рассматриваемого примера показан на рис. 3.4, б.

Далее контролируется соответствие заданных междупутий $\mathbf{m} \in \Omega_{\mathrm{Bx}}$ ребрам дерева $\bar{D}$. В случае обнаружения несоответствия выдается сообщение об ошибке.

В заключение определяются действительные значения ординат $Y_{i}$ всех горизонтальных путей схемы, для чего должна быть задана ордината опорной точки $Y_{0}$, находящейся на одном из этих путей. Для расчета ординат $Y_{i}$ необходимо построить ориентированное дерево $D$ (см. п. 2.2.2), принимая вершину остова $\bar{D}$, соответствующую пути с заданной ординатой $Y_{0}$, в качестве корня. На рис. 3.5 показано дерево, полученное из остова $\bar{D}$ (см. рис. $3.4,6)$, когда в качестве корня взята вершина $w_{2}(r=2-$ путь 2$)$; в узлах дерева указаны номера путей, а дугам поставлены в соответствие междупутья.

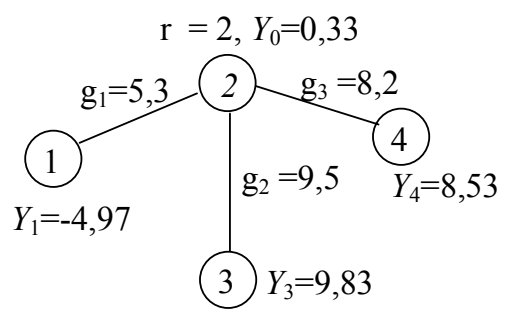

Рисунок 3.5 - Дерево горизонтальных путей $D=(W, H)$ для станции А Для определения значений ординат $Y_{i}$ используется процедура обхода дерева $D$ в глубину. При этом ордината очередного пути определяется как 


$$
Y_{j}=Y_{i}+g_{i j} \operatorname{sign}\left(y\left(w_{i}\right)-y\left(w_{j}\right)\right)
$$

Изложенные процедуры позволяют выполнить автоматизированное преобразование входной модели станции и получить модель ее горизонтальных путей, необходимую для расчета ординат всех точек плана станции.

\section{3. Преобразование входной модели станции в каноническую}

Процесс преобразования входной модели во внутреннюю выполняется автоматически и связан с решением следующих задач: формирование списков инцидентности ориентированного графа $G$; определение направления отклонения путей стрелочных переводов и классификация примыкающих участков; определение направления поворота круговых кривых.

\subsection{1. Формирование списков инцฺидентности вериин орграфа станщฺии}

Для формирования списков инцидентности выполняется анализ множества участков путей станции $е \in \Omega_{\text {вх }}$, которые соответствуют дугам графа $G$. Конечные точки участков путей сортируются таким образом, чтобы $x_{v}<x_{u}$. На основе анализа списка дуг графа $G$ составляется список его вершин $\left(v_{1}, v_{2}, \ldots\right.$, $\left.v_{n}\right)$, в котором каждая из них представлена структурой:

$$
v_{i}=\left(v_{j}, v_{l}, d^{+}\left(v_{i}\right), d^{-}\left(v_{i}\right), x_{i}, y_{i}\right)
$$

где $v_{j}, v_{l}$ - конечные вершины дуг, исходящих из $v_{i}$ (число таких вершин равно $\left.d^{+}\left(v_{i}\right) \leq 2\right)$;

$d^{+}\left(v_{i}\right), d^{-}\left(v_{i}\right)$ - соответственно, полустепени исхода и захода вершины $v_{i}$ (могут принимать значения 0,1 или 2 в зависимости от типа вершины);

$x_{i}, y_{i}-$ координаты вершины $v_{i}$ на эскизе.

Для определения типа вершины $v_{i}$ (ЦП, ВУП, КП) вычисляется параметр $t_{i}$ :

$$
t_{i}=3 \cdot d^{-}\left(v_{i}\right)+d^{+}\left(v_{i}\right)
$$


При этом полученным значениям $t_{i}$ соответствуют следующие типы вершин: $t_{i}=1-$ левый КП $\left(v_{i} \in V^{W}\right) ; t_{i}=3-$ правый КП $\left(v_{i} \in V^{W}\right) ; t_{i}=4-$ ВУП $\left(v_{i} \in V^{C}\right) ; t_{i}=5$ - ЦП противошерстной стрелки $\left(v_{i} \in V^{S}\right) ; t_{i}=7$ - ЦП пошерстной стрелки $\left(v_{i} \in V^{S}\right)$. Значения $t_{i}=2,6$ или 8 недопустимы и свидетельствуют о наличии ошибок в эскизе.

В зависимости от найденного типа вершины $v_{i}$ ей присваивается уникальный текущий номер $N_{i}$ из диапазона номеров, отведенных для данного типа вершины: $N_{i}=\{1,99\}, v_{i} \in V^{S}, N_{i}=\{101,199\}, v_{i} \in V^{W}, N_{i}=\{201,299\}, v_{i} \in V^{C}$. Далее осуществляется анализ множества объектов $\mathbf{s} \in \Omega_{\mathrm{Bx}}$ и $\mathbf{c} \in \Omega_{\mathrm{Bx}}$ и идентификация по координатам соответствующих им вершин графа $G$. Списки параметров вершин $X^{S}$ и $X^{C}$ дополняются параметрами соответствующих объектов и исходящих ребер $X^{E}$ (данные о величине вставок $f_{\mathrm{P}}, f_{\mathrm{B}}$ и номере горизонтального пути $w)$.

\subsection{2. Определение направления боковых путей стрелочных переводов}

Если вершина является ЦП, то для несимметричных стрелочных переводов при любой укладке стрелочного перевода необходимо определить его направление (лево- или правосторонний). Кроме того, если вершина соответствует противошерстному стрелочному переводу (рамные рельсы слева от ЦП, см. рис. 3.6), возникает задача классификации упорядочения смежных вершин в списке инцидентности. Для примера рассмотрим вершину v. Обозначим дуги, инцидентные вершине $v$, соответственно, буквами: $\delta$ - дугу, заходящую в вершину $v, \delta_{1}$ и $\delta_{2}-$ дуги, исходящие из нее. При этом одна из дуг $\left(\delta_{1}\right)$ является продолжением дуги $\delta$, другая $\left(\delta_{2}\right)-$ отклоняется от нее влево (рис. 3.6, a) или вправо (рис. 3.6, б) в зависимости от направления стрелочного перевода. Конечным вершинам $v_{1}, v_{2}$, соответственно, дуг $\delta_{1}$ и $\delta_{2}$ в процессе построения модели были присвоены номера $N_{1}$ и $N_{2}$, которые и образуют список инцидентности для вершины $v$ с номером $N$. 


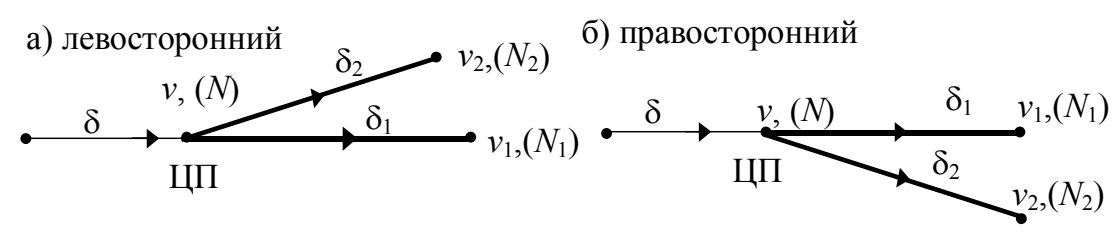

Рисунок 3.6 - Схема расположения дуг, инцидентных противошерстному стрелочному переводу (вершина $v$ )

Вершины $v_{1}, v_{2}$ включают в структуру (3.5) в порядке их появления в списке дуг, который, в свою очередь, зависит от последовательности вычерчивания на экране дисплея элементов схемы. В результате требуемый порядок записи номеров вершин в списке инцидентности $\left(N_{P}=N_{1}, N_{B}=N_{2}\right)$ может быть нарушен. Так, для приведенного на рис. 3.6 случая в структуре (3.5) вершины должны быть записаны в порядке $\left(v_{1}, v_{2}\right)$; иначе порядок номеров в списке инцидентности для вершины $v$ необходимо изменить.

При указании в списке инцидентности $\left(N_{P}, N_{B}\right)$ вершины, соответствующей пошерстному стрелочному переводу (рамные рельсы справа от ЦП), он должны быть отмечен в зависимости от направления примыкания (к прямому или боковому пути крестовины).

Таким образом, для обыкновенных стрелочных переводов возникает задача определения сторонности стрелочного перевода и классификации соответствующих ему путевых участков на участок рамного рельса, прямого и бокового пути крестовины. Для симметричных стрелочных переводов необходимо классифицировать участки на участок рамного рельса, левого и правого пути крестовины. Следует заметить, что в процессе ввода немасштабной схемы возможны искажения, в результате которых ни один из путей стрелочного перевода $C B$ и $C D$ не является в точности продолжением рамного рельса $A C$ (см. рис $3.76,6$ ).

Направление укладки перевода и дуга графа, соответствующая рамному рельсу, определяется по полустепени исхода вершины: если $d^{+}(v)=1-$ перевод противошерстный, а рамному рельсу соответствует исходящая дуга; 
если $d^{+}(v)=2$ - перевод пошерстный, а рамному рельсу соответствует заходящая дуга.
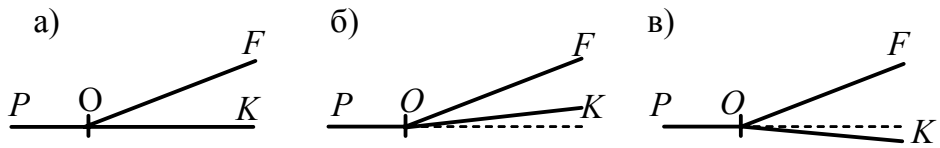

Рисунок 3.7 - Изображения обыкновенных стрелочных переводов на немасштабной схеме: $a$ - без искажений; $\sigma, \varepsilon-$ с искажениями

Направление отклонения путей стрелочных переводов $s$ (левосторонний $-s=0$, правосторонний $-s=1$ ) определяется по положению точек $F$ и $K$ относительно направления рамного рельса (ориентированного отрезка $P O$ ). Для определения положения некоторой точки $A$ относительно ориентированного отрезка $B C$ может быть преобразовано выражение (3.3) [28]:

$$
Z_{A}^{B C}=\operatorname{sign}\left(\left(y_{A}-y_{B}\right)\left(x_{C}-x_{B}\right)-\left(y_{C}-y_{B}\right)\left(x_{A}-x_{B}\right)\right),
$$

Величина $Z$ принимает значение -1, если точка лежит справа от отрезка, значение 1 , если точка лежит слева от отрезка, и 0 , если точка и отрезок принадлежат одной прямой. Таким образом, если $Z_{F}^{P O}+Z_{K}^{P O}<0$, то стрелочный перевод правосторонний $(s=1)$; если $Z_{F}^{P O}+Z_{K}^{P O}>0$, то стрелочный перевод левосторонний $(s=0)$.

Если $Z_{F}^{P O}+Z_{K}^{P O}=2$ или $Z_{F}^{P O}+Z_{K}^{P O}=-2$ (см. рис. 3.7, б) для классификации путевых участков необходимо с помощью выражения (3.7) установить положение точки $F$ относительно отрезка $O K$. При этом, если $\operatorname{sign}\left(F_{F}^{O K}\right)=\operatorname{sign}\left(F_{F}^{P O}\right)$, то отрезок $O F$ соответствует боковому пути перевода, иначе он соответствует прямому пути стрелочного перевода.

Если $Z_{F}^{P O}+Z_{K}^{P O}=0$ (см. рис. $\left.3.7,8\right)$, то направление отклонения бокового пути перевода определяется по результатам сравнения величины углов отрезков $P O F$ и $P O K$. Указанная задача может быть решена на основании сравнения угловых коэффициентов отрезков $O F$ и $O K$ с угловым коэффициентом отрезка PO. Однако такой поход связан с необходимостью дополни- 
тельного логического контроля для вертикальных отрезков, поэтому указанную задачу целесообразно решать методами векторной алгебры.

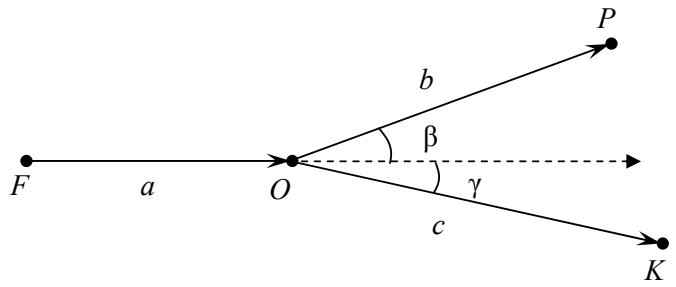

Рисунок 3.8 - Векторное представление изображения стрелочного перевода

Для решения этой задачи необходимо определить, какой из векторов $(b$ или $c$ ) имеет больший угол наклона к направлению вектора $a$ (см. рис. 3.8). С этой целью должно быть вычислено значение $z=\operatorname{sign}(\beta-\gamma)$; при этом если $z>0$, то боковому пути соответствует отрезок $O P$, а если $z<0$, то боковому пути соответствует отрезок $O K$. Учитывая, что после решения задачи определения направления укладки стрелочного перевода (см. выше) углы $\beta$ и $\gamma$ могут принимать значения в диапазоне $\left(+\frac{\pi}{2},-\frac{\pi}{2}\right)$, то величина $z$ может быть определена и из выражения $z=\operatorname{sign}(\cos \gamma-\cos \beta)$. Значение $(\cos \gamma-\cos \beta)$ можно получить методами векторной алгебры:

$$
\cos \gamma-\cos \beta=\frac{\bar{a} \cdot \bar{c}}{|a| \cdot|c|}-\frac{\bar{a} \cdot \bar{b}}{|a| \cdot|b|}=\frac{\bar{a} \cdot \bar{c} \cdot|b|-\bar{a} \cdot \bar{b} \cdot|c|}{|a| \cdot|b| \cdot|c|}=\frac{\bar{a}}{|a| \cdot|b| \cdot|c|}(\bar{c} \cdot|b|-\bar{b} \cdot|c|),
$$

откуда

$$
z=\operatorname{sign}\left[\frac{\bar{a}}{|a| \cdot|b| \cdot|c|}(\bar{c} \cdot|b|-\bar{b} \cdot|c|)\right]
$$

Ввиду того, что $|a| \cdot|b| \cdot|c|>0$, выражение (3.8) можно упростить:

$$
z=\operatorname{sign}[\bar{a} \cdot(\bar{c} \cdot|b|-\bar{b} \cdot|c|)]
$$


В координатах указанное выражение принимает вид:

$$
z=\operatorname{sign}\left[x_{a}\left(|b| \cdot x_{c}-|c| \cdot x_{b}\right)+y_{a}\left(|b| \cdot y_{c}-|c| \cdot y_{b}\right)\right]
$$

где $x_{a}=x_{O}-x_{F}, y_{a}=y_{O}-y_{F} ; x_{b}=x_{P}-x_{O}, \quad y_{b}=y_{P}-y_{O} ; \quad x_{c}=x_{K}-x_{O}$, $y_{c}=y_{K}-y_{O} ;|b|=\sqrt{x_{b}^{2}+y_{b}^{2}} ;|c|=\sqrt{x_{c}^{2}+y_{c}^{2}}$.

По результатам выполненных вычислений в необходимых случаях корректируются номера инцидентных вершин $N_{P}$ и $N_{B}$ в (3.5), а также значения соответствующих им параметров $f_{P}, f_{B}$.

Для симметричных стрелочных переводов на основании анализа степеней захода и исхода определяется отрезок, соответствующий направлению укладки рамных рельсов, а далее устанавливается положение точки $F$ отно-

сительно отрезка $O K$ с помощью выражения (3.7). При этом, если $Z_{F}^{O K}=1$, то отрезок $O F$ соответствует левому пути симметричного стрелочного перевода, иначе $O F$ соответствует правому пути.

\subsection{3. Определение направления поворота круговых кривых}

Направление поворота круговых кривых во внутренней модели определяется знаком угла. Для удобства ввода входная модель содержит только абсолютное значение величины угла поворота $|\alpha|$, а его знак необходимо установить в результате анализа немасштабной схемы. Возможные случаи ориентации кривых изображены на рис. 3.9.

Направление угла поворота устанавливается на основании определения положения точки $A$, соответствующей конечной вершине исходящей дуги, относительно отрезка $B C$, соответствующего заходящей дуге, с помощью выражения (3.7); при этом значение угла определяется как $\alpha=\operatorname{sign}\left(Z_{A}^{B C}\right) \cdot|\alpha|$.

В результате указанных действий входная модель преобразовывается во внутреннюю модель (см. табл. 2.3, 2.4). 

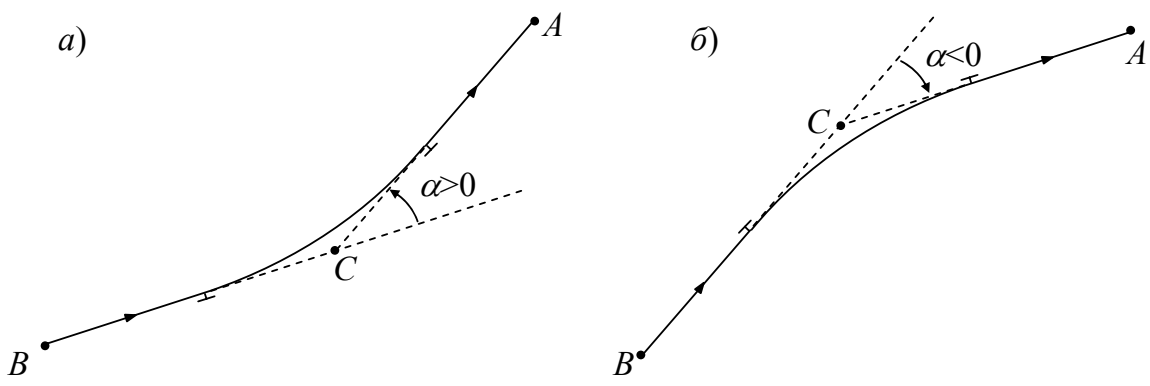

Рисунок 3.9 - Векторное представление изображения угла поворота кривой: $a$ - поворот против часовой стрелки; $\sigma$ - поворот по часовой стрелке

\subsection{4. Формирование списка сигналов на плане станщии}

Целью формирования списка сигналов станции является преобразование описания сигнала в виде структуры $\mathbf{q}_{\text {вх }}(2.6)$ во входной модели в описание $\mathbf{q}_{\text {вн }}$ (2.7). Для этого выполняется поиск ближайшей дуги графа $G$, располагающейся слева от сигнала. Расстояние $l_{\mathbf{p}_{q}}^{\mathbf{e}_{i}}$ от сигнала до $i$-й дуги графа определяется с помощью выражения (3.3). Учитывая, что знак $l_{\mathbf{p}_{q}}^{\mathbf{e}_{i}}$ указывает на положение точки относительно ориентированного отрезка, выбор дуги осуществляется с помощью выражения

$$
\begin{aligned}
& \left\{\begin{array}{l}
d_{q}=\frac{1-\operatorname{sign}\left(l_{\mathbf{p}_{q}}^{\mathbf{e}_{i}}\right)}{2} \\
0 \leq \frac{\left(\mathbf{p}_{\mathrm{\kappa} i}-\mathbf{p}_{\mathrm{н} i}\right)\left(\mathbf{p}_{q}-\mathbf{p}_{\mathrm{н} i}\right)}{\left|\mathbf{p}_{\mathrm{\kappa} i}-\mathbf{p}_{\mathrm{Hi} i}\right|^{2}} \leq 1,
\end{array}\right. \\
& l_{\mathbf{p}_{r}}^{\mathbf{e}_{i}}=\min , i=1,2, \ldots, m
\end{aligned}
$$

где $\mathbf{p}_{\text {нi}}, \mathbf{p}_{\text {кi }}-$ точки, соответствующие начальной и конечной вершинам дуги $\mathbf{e}_{i}$.

Далее на графе $G$ в направлении действия сигнала составляется цепь дуг, начинающаяся выбранной дугой $\mathbf{e}_{i}$ и заканчивающейся дугой, у которой конечной вершиной является стрелочный перевод (см. рис. 3.10). 


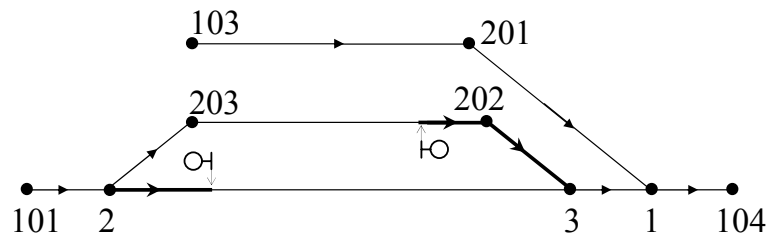

Рисунок 3.10 - Идентификация стрелочного перевода, у которого установлен сигнал

Номер найденного стрелочного перевода $v$ и относительное положение сигнала $z$ сохраняются в структуре $\mathbf{q}_{\text {вн }}(2.7)$. 


\section{ГЛАВА 4}

\section{РАСЧЕТ ПАРАМЕТРОВ ПЛАНА ПУТЕВОГО РАЗВИТИЯ СТАНЦИЙ}

\section{1. Общие принципы расчета}

Методика расчета элементов плана путевого развития станции основана на определении координат всех точек каждого базового элемента в локальной системе $\Sigma_{k}$ и последующем преобразовании координат отдельных точек для определения параметров кривых, сопрягающих пути смежных базовых элементов [26].

Расчет координат точек отдельного базового элемента производится в системе $\Sigma_{k}$, в которой основная группа путей параллельна оси абсцисс $O x_{k}$.

Расчет производится в следующем порядке. Первоначально определяются ординаты точек, находящихся на путях, параллельных оси абсцисс; при расчетах используются данные о междупутьях; привязка осуществляется через ординату заданной опорной точки $y_{0}$. Ординатам остальных точек, а также абсциссам всех точек, кроме опорной, присваиваются неопределенные значения $\left(y_{i}=\infty, x_{i}=\infty\right)$.

Далее определяются углы наклона $\theta_{j}$ к оси абсцисс всех отрезков базового элемента. С этой целью осуществляется классификация отрезков на горизонтальные и наклонные по разности ординат их концов; если ординаты неизвестны $\left(y_{i}=\infty\right)$, то отрезок относится к наклонным. На базе полученных данных осуществляется определение углов наклона $\theta_{j}$ отрезков, смежных с $i$ ой вершиной графа $G$. Если $v_{i} \in V^{S}$ (вершина $v_{i}$ является центром перевода), то определяется число отрезков $n_{\text {ні }}$ с неизвестным углом наклона, примыкающих к стрелке $\left(n_{\text {нi }} \in[0,3]\right)$. Далее определяется полустепень исхода вершины $d^{+}\left(v_{i}\right)$, значение которой характеризует направление укладки перевода (пошерстный $-d^{+}\left(v_{i}\right)=1$, противошерстный $-d^{+}\left(v_{i}\right)=2$ ). При этом контролируется достоверность модели $\left(\operatorname{deg} v=d^{+}\left(v_{i}\right)+d^{-}\left(v_{i}\right)=3\right)$ и составляются 
списки $k_{i}=d^{+}\left(v_{i}\right)$ исходящих и $m_{i}=d^{-}\left(v_{i}\right)$ заходящих в вершину $v_{i}$ дуг. Параметры $n_{\text {нi }}$ и $\operatorname{deg}^{+}\left(v_{i}\right)$ определяют выбор алгоритма расчета неизвестных углов наклона отрезков, примыкающих к стрелочному переводу (вершине $v_{i}$ ); в расчетах учитываются величина и знак угла крестовины (для правосторонних стрелок $\alpha<0)$. В случае, если у стрелочного перевода неизвестны углы наклона всех примыкающих отрезков $\left(n_{\text {нi }}=3\right)$, расчеты не производятся; они будут выполнены после нахождения углов наклона некоторых из этих отрезков при рассмотрении вершин, смежных с $v_{i}$.

Для вершин углов поворота $\left(v_{i} \in V^{C}\right)$ с известной величиной $\beta$ также определяется число примыкающих отрезков $n_{\text {нi }}$ с неизвестным углом наклона $\left(n_{\text {нi }} \in[0,2]\right)$. В случае, если $n_{\text {нi }}=1$, определяется угол наклона неизвестного отрезка по известному углу наклона другого отрезка и углу поворота кривой $\beta$. Если же угол $\beta$ неизвестен, либо $n_{\text {нi }} \neq 1$, расчет для вершины $v_{i}$ не производится.

После расчета углов наклона отрезков определяются параметры кривых (угол поворота $\beta$, тангенс $T$, длина $K$ ). Угол поворота $\beta$ определяется как разность углов наклона отрезков, примыкающих к его вершине; по абсолютной величине рассчитанного значения $|\beta|$ определяются тангенс $T$ и длина кривой $K$.

Рассчитанные параметры дополняют внутреннюю модель станции, после чего на ее базе осуществляется идентификация элементов плана станции и расчет расстояний $l_{j}$ и вставок $f_{j}, j=1,2, \ldots, n$ между его характерными точками (см. п. 4.2). Далее осуществляется расчет ординат $Y_{i}$ точек, не лежащих на горизонтальных путях $\left\{Y_{i}: w_{i}=0, i=1, \ldots, m\right\}$. Для расчета орграф $G$ преобразуется в неориентированный граф $\bar{G}$, на котором строится остовное дерево с использованием алгоритма поиска в глубину. Дерево строится таким образом, чтобы в его вершинах были точки с известными или рассчитанными в процессе построения ординатами. Концевыми являются вершины $v_{i} \in V^{W}$ 
(концы путей), либо вершины, инцидентные ребра которых имеют неопределенный вес (т.е. неизвестное приращение ординаты $\Delta Y$ ) и не позволяют продолжить построение дерева. В целях сокращения объема вычислений построение дерева прекращается, когда для всех вершин графа $G$ ординаты $Y$ станут известными.

После расчета ординат $Y$ осуществляется анализ углов наклона отрезков $\theta_{j}$, поставленных в соответствие дугам графа; при этом могут быть обнаружены ребра с неопределенным значением $\theta_{j}\left(\theta_{j}=\infty\right)$. Это свидетельствует о наличии в рассмотренной схеме таких углов поворота, величины которых нельзя определить как суммы углов соответствующих стрелочных переводов. Подобный случай может иметь место, например, при наличии в схеме сокращенных соединений путей. В таком случае осуществляется расчет одного из неизвестных углов поворота по разработанной методике (см. п. 4.4), после чего выполняется установка первоначальных значений координат всех вершин графа $G$ и вставок между ними и повторяются расчеты всех элементов плана. Указанное повторение необходимо, так как вычисленное на очередном этапе расчетов значение неизвестного угла $\beta$ влияет на параметры связанных с ним элементов; повторение продолжается до тех пор, пока не будут определены наклоны $\theta_{j}$ всех отрезков схемы.

На заключительном этапе расчетов вычисляются длины всех наклонных отрезков по разности ординат их концов:

$$
l_{j}^{*}=\frac{\left|Y_{v}-Y_{u}\right|}{\sin \theta_{j}}
$$

При этом контролируются условия определенности указанных ординат $Y_{v} \neq \infty, Y_{u} \neq \infty$. Также контролируются возможные отличия рассчитанных длин $l_{j}^{*}$ от значений, установленных на предыдущих этапах вычислений; указанные различия будут иметь место в ряде случаев, один из которых для примера приведен на рис. 4.1 . 


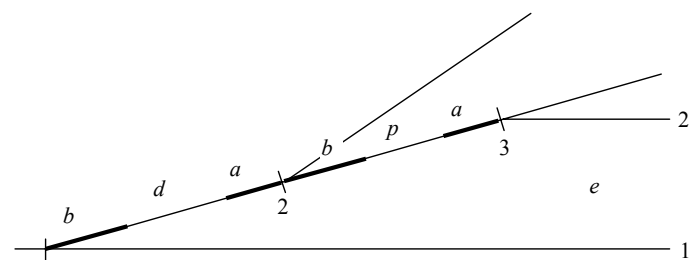

Рисунок 4.1 - Схема корректирования конструктивных вставок между стрелочными переводами 1-2-3

Как следует из приведенного рисунка, каждая из вставок 1-2 и 2-3, рассматриваемая изолированно от всей схемы, представляет собой конструктивную вставку при попутной укладке переводов 1-2-3. В то же время в данной схеме их суммарная длина должна соответствовать расстоянию между осями путей 1 и 2. Учитывая данное обстоятельство, величина $l_{j}^{*}(4.1)$, рассчитанная по разности ординат смежных точек наклонного отрезка, имеет приоритет пред присвоенным ранее конструктивным значением $l_{j}$ и принимается как окончательное значение. Следует добавить, что для определенности выбора расчетной и конструктивной вставок в схеме, приведенной на рис. 4.1, необходимо в модели расчетную вставку пометить кодом 9999.

По завершении определения и контроля вставок на наклонных отрезках осуществляется расчет абсцисс $X_{i}$ всех точек плана. Методика расчета также основана на построении на неориентированном графе $\bar{G}$ остовного дерева с корнем в опорной точке; при этом, как и для расчета ординат вершин, используется алгоритм поиска в глубину. При построении дерева одновременно осуществляется контроль связности графа, поскольку при ее отсутствии невозможно определить координаты всех точек плана.

После построения дерева осуществляется расчет расстояний и вставок между точками, являющимися концевыми вершинами ребер графа $\bar{G}$, не вошедших в остовное дерево.

В заключение осуществляется расчет ординат всех концевых вершин графа $G$ (вершин, являющихся концами путей $v_{i} \in V^{W}$ ). При этом все отрез- 
ки, для которых во входной модели не задана конкретная длина, выравниваются, соответственно, по левой $\left(d^{+}\left(v_{i}\right)=1\right)$ или по правой $\left(d^{+}\left(v_{i}\right)=0\right)$ границе плана. После выравнивания отрезков осуществляется расчет координат вершин $v_{i} \in V^{W}$ и определяются длины вставок, находящихся на инцидентных им отрезках.

Рассчитанные таким образом параметры используются для построения выходной модели станции (см. п. 2.4) и визуализации графического изображения ее плана на экране дисплея.

\section{2. Расчет расстояний между точками}

Расстояния между смежными точками схемы (длины отрезков и прямые вставки) зависят, в первую очередь, от типа точек, ограничивающих отрезки (ЦП, ВУ, КП). Возможны 5 различных комбинаций указанных точек на концах отрезка: ЦП-ЦП, ЦП-ВУ (ВУ-ЦП), ЦП-КП (КП-ЦП), ВУ-ВУ, ВУ-КП (КП-BУ).

Наиболее обширный класс составляют отрезки, ограниченные двумя стрелочными переводами (ЦП-ЦП). Расстояние между двумя смежными ЦП состоит из трех элементов:

$$
l_{j k}=d_{j}+f_{j k}+d_{k}
$$

где $d_{j}, d_{k}-$ геометрические размеры $a, b$ стрелочных переводов;

$f_{j k}-$ прямая вставка.

Геометрические размеры стрелочных переводов известны и поэтому задача расчета расстояния сводится к нахождению вставки, которая определяется их взаимным расположением. С этой целью все множество вариантов взаимного расположения стрелочных переводов разделено на ряд принципиальных схем, различающихся методом определения указанной прямой вставки. При этом 9 из них образуются при соединении двух переводов, у которых боковые пути отклоняются в одну сторону - двух правосторонних (ПП) или двух левосторонних (ЛЛ) переводов. Еще 9 вариантов возможны при соеди- 
нении разносторонних переводов - ПЛ или ЛП. Указанные 18 вариантов могут быть сведены к 6 принципиально различным схемам (см. рис. 4.2).

a)

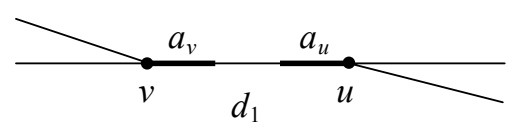

8)

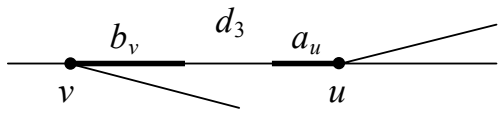

$\partial)$

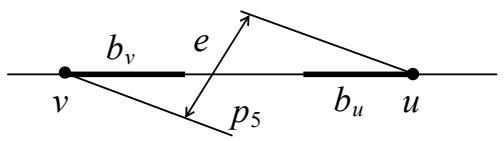

б)

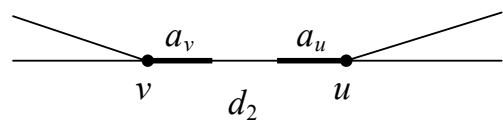

2)

Схема 4

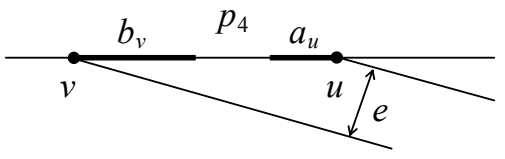

e)

Схема 6

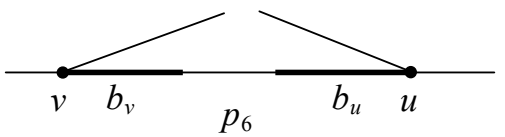

Рисунок 4.2 - Схемы взаимного расположения смежных стрелочных переводов

Схема 1 (рис. 4.2, a) - встречная укладка переводов с боковыми путями, направленными в разные стороны. Стрелочные переводы расположены остряками навстречу друг другу; между стыками рамных рельсов укладывается конструктивная вставка $d$.

Схема 2 (рис. 4.2, б) - встречная укладка переводов с боковыми путями, направленными в одну сторону. Стрелочные переводы расположены остряками навстречу друг другу; между стыками рамных рельсов укладывается конструктивная вставка $d$.

Схема 3 (рис. 4.2, в) - попутная укладка переводов с боковыми путями, направленными в разные стороны. Между торцом крестовины одного стрелочного перевода и стыком рамного рельса другого укладывается конструктивная вставка $d$. 
Схема 4 (рис. 4.2, г) - попутная укладка переводов с параллельными боковыми путями, направленными в одну сторону. Между торцом крестовины одного стрелочного перевода и стыком рамного рельса другого укладывается расчетная вставка $p$, величина которой зависит от расстояния между осями отклоняющихся путей.

Схема 5 (рис. 4.2, ) - встречная укладка переводов с параллельными боковыми путями, направленными в разные стороны. Между торцами крестовин стрелочных переводов укладывается расчетная вставка $p$, величина которой зависит от расстояния между осями отклоняющихся путей.

Схема 6 (рис. 4.2, e) - встречная укладка переводов с боковыми путями, направленными в одну сторону. Между торцами крестовин переводов укладывается расчетная вставка $p$, величина которой зависит от особенностей конструкции соединения путей, в котором используется данная схема. Чаще всего схема 6 встречается в перекрестных съездах (рис. 4.3, a), а также в замкнутых контурах (рис. 4.3, б).

a)

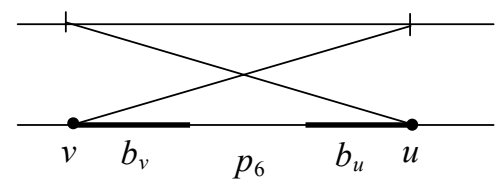

б)

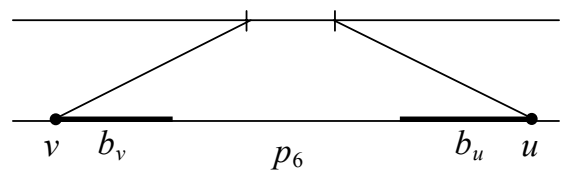

Рисунок 4.3 - Варианты использования схемы 6 расположения стрелочных переводов на планах станций: $a$ - перекрестный съезд; $\sigma$ - замкнутый контур

Если хотя-бы один из концов отрезка является концом пути или вершиной угла, то такой отрезок относится к схемам 7-9 (рис. 4.4).

Схема 7 (рис. 4.4, $a, 6$ ) - хотя-бы один из концов отрезка является концом пути; при этом вторым концом может быть как центр перевода, так вершина угла.

Схема 8 (рис. 4.4, в) - один из концов отрезка является центром перевода, а другой - вершиной угла.

Схема 9 (рис. 4.4, г) - оба конца отрезка являются вершинами углов. 
a)

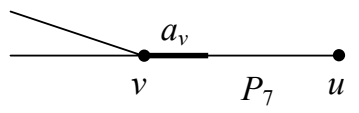

в)

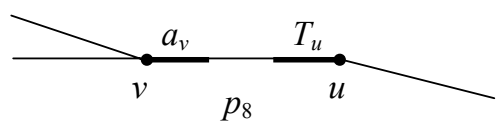

б)

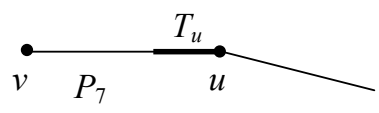

2)

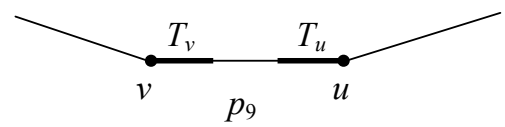

Рисунок 4.4 - Схемы отрезков, у которых хотя бы один из концов является стрелочным переводом

Предложенная классификация отрезков достаточно эффективна и позволяет определять как необходимую длину прямой вставки, так и общую длину каждого отрезка.

Как известно, вставки между стрелочными переводами делятся на конструктивные и расчетные. Конструктивные вставки (схемы 1-3) определяются по нормативным таблицам в зависимости от типа принципиальной схемы, категории пути, условий укладки и допустимой скорости движения поездов по стрелочным переводам [1].

Расчетные вставки (схемы 4, 5) определяются, как правило, из условия обеспечения необходимого расстояния $g_{j k}$ между осями параллельных путей:

$$
f_{j k}=\frac{g_{j k}}{\sin \alpha}-\left(d_{j}+d_{k}\right)
$$

где $\quad \alpha-$ угол крестовины.

Возможны два различных варианта определения расстояния $g_{j k}$. В первом случае стрелочные переводы $j$ и $k$ находятся на разных горизонтальных путях с известными ординатами, соответственно $Y_{j}$ и $Y_{k}$; при этом $g_{j k}=\left|Y_{j}-Y_{k}\right|$. Во втором случае, когда стрелочные переводы находятся на одном горизонтальном пути, расстояние $g_{j k}$ должно быть задано в явном виде во входной модели горловины; в противном случае при расчетах будет принято стандартное значение $g_{j k}=5,3$ м. Если же стрелочные переводы $j$ и $k$ находят- 
ся на наклонном пути, а расстояние $g_{j k}$ или непосредственно вставка $f_{j k}$ не задано, то величина $f_{j k}$ находится по разности ординат точек $j$ и $k$ и углу наклона этого пути к оси абсцисс

$$
f_{j k}=\frac{\left|Y_{j}-Y_{k}\right|}{\sin \theta_{j k}}-\left(d_{j}+d_{k}\right) .
$$

При этом предполагается, что схема горловины и набор заданных параметров позволяют найти ординаты $Y_{j}$ и $Y_{k}$.

Для отрезков, отнесенных к схеме 6, расчет зависит от величины $p_{j k}$, заданной во входной модели. Если вставка $p_{j k}$ задана явно, то ее числовое значение используется для расчета общего расстояния $l_{j k}$ между точками $j$ и $k$ (4.2). Если $p_{j k}=9999$, то соответствующая вставка определяется по разности координат точек $j$ и $k$, по формулам (4.4) - (4.5), приведенным ниже. Если же $p_{j k}=0$, то эти стрелочные переводы идентифицируются как части перекрестного съезда; при этом вставка $f_{j k}$ определяется как

$$
f_{j k}=\frac{e}{\operatorname{tg} \alpha}-\left(b_{j}+b_{k}\right) .
$$

При этом одновременно определяются вставки $f_{\text {гп }}$ съезда, проходящего через глухое пересечение:

$$
f_{\text {гп }}=\frac{1}{2}\left(\frac{e}{\sin \alpha}-\left(2 b+l_{\text {гा }}\right)\right),
$$

где $\quad l_{\text {гп }}-$ длина глухого пересечения.

В отдельных случаях, когда, например, вставка, относящаяся к любому типу схемы, замыкает некоторый контур, ее величина определяется по разности координат концов соответствующего отрезка:

$$
f_{j k}=\frac{\Delta x_{j k}}{\cos \theta_{j k}}-\left(d_{j}+d_{k}\right)
$$

или

$$
f_{j k}=\frac{\Delta y_{j k}}{\sin \theta_{j k}}-\left(d_{j}+d_{k}\right)
$$

где $\theta_{j k}-$ угол наклона отрезка $l_{j k}$ к оси абсцисс. 
В этих случаях расчет вставки откладывается до окончания расчета соответствующих координат. Учитывая, что выбор вставки, определяемой из условия замкнутости контура, может быть неоднозначным, соответствующие дуги во входной модели должны быть помечены кодом 9999.

Отдельные вставки могут быть заданы явно во входной модели; в частности может быть задана длина расчетного пути, определяющего полезную длину всех путей парка. Известная длина расчетного пути обеспечивает возможность перехода из одной горловины парка в другую при расчетах координат. Обычно такой случай имеет место, когда станция (парк) расположена на прямой и может быть описана единственным графом $G$. В случаях, когда вставка задана, определяется только общая длина отрезка (4.2).

Прямые вставки на отрезках, ограниченных точками ЦП-ВУ, определяются либо из конструктивных соображений, либо из условия обеспечения требуемого междупутья $g$ :

$$
f_{j k}=\frac{g_{j k}}{\sin \alpha_{j}}-\left(d_{j}+T_{k}\right)
$$

где $T_{k}$ - тангенс кривой.

В случаях, когда отрезок ограничен двумя ВУ, вставка определяется, как правило, по разности координат его концов:

$$
f_{j k}=\frac{\Delta x_{j k}}{\cos \theta_{j k}}-\left(T_{j}+T_{k}\right)
$$

или

$$
f_{j k}=\frac{\Delta y_{j k}}{\sin \theta_{j k}}-\left(T_{j}+T_{k}\right) .
$$

Наконец, в случаях, когда один из концов отрезка является концом пути КП, соответствующая вставка либо должна быть задана, либо она будет определена по границе станции. 


\section{3. Расчет координат точек}

Расчет производится после определения всех вставок; для расчета используются рекуррентные формулы:

$$
\begin{aligned}
& x_{k}=x_{j}+l_{j k} \cos \theta_{j k} . \\
& y_{k}=y_{j}+l_{j k} \sin \theta_{j k}
\end{aligned}
$$

Расчет начинается от опорной точки $\mathrm{P}_{0}$, координаты которой должны быть заданы: $P_{0}=\left(X_{0}, Y_{0}\right)_{\Sigma k}$

Рациональная последовательность расчета координат достигается при использовании предлагаемой ниже методики. Для расчета координат исходный ориентированный граф $G=(V, E)$ целесообразно преобразовать в неориентированный, который будем обозначать как $\bar{G}=(V, \bar{E})$. При этом необходимо удалить часть дуг графа $G$, так чтобы в получившемся подграфе $\bar{G}_{\mathrm{o}}=\left(V, \bar{E}_{\mathrm{o}}\right)$ не было циклов. Другими словами, нужно из графа $G$ построить остовное дерево $U=\left(V, E_{U}\right)$ с корнем в опорной точке (вершина $\left.v_{0}\right)$. Действительно, наличие цикла в графе может привести к неоднозначности расчета координат некоторой вершины $v$, к которой существует более одного маршрута от вершины $v_{0}$. Очевидно, что удалены должны быть помеченные дуги графа $G$, для которых не были рассчитаны на первом этапе длины и прямые вставки. Обычно это отрезки, замыкающие некоторые контуры в схеме и помеченные во входной модели кодом 9999, либо отрезки, относящиеся к схемам 8,9 , если для них не заданы в явном виде длины прямых вставок.

Учитывая возможные ошибки в исходных данных, после построения остовного подграфа $U$ графа $G$, необходимо определить число его связных компонентов (оно должно равняться 1) и убедится в отсутствии в нем циклов. После того, как будет построено остовное дерево $U$, необходимо организовать обход всех его вершин и ребер для непосредственного расчета координат. Наиболее подходящим для решения данных задач является алгоритм поиска в глубину, который характеризуется вычислительной сложностью порядка $O(n+m)$. 
Для представления неориентированного графа $\bar{G}=(V, \bar{E})$ в памяти ЭВМ использованы списки ребер $\bar{E}=\left(\bar{e}_{1}, \bar{e}_{2}, \ldots, \bar{e}_{m},\right)$, где $m$ - число ребер графа $G$. Поскольку ребро графа можно хранить, используя две ячейки (по одной на каждую концевую вершину), то для хранения всего списка $\bar{E}$ достаточно $2 m$ ячеек. Кроме того, для реализации алгоритма поиска в глубину и выполнения указанных выше проверок необходимо список ребер $\bar{E}$ дополнить списком пометок ребер $\Xi=\left(\xi_{1}, \xi_{2}, \ldots, \xi_{m}\right)$, с помощью которых отмечаются также удаленные дуги исходного графа $G$.

Для организации вычисления координат точек базового элемента был разработан алгоритм поиска в глубину, основанный на выбранном методе представления графа в ЭВМ и учитывающий особенности задачи.

Остовное дерево для расчета абсцисс $X_{i}$, построенное для рассмотренного примера станции (см. рис. 2.1), приведено на рис. 4.5 .

После построения дерева осуществляется расчет расстояний и вставок между точками, являющимися концевыми вершинами ребер графа $\bar{G}$, не вошедших в остовное дерево; в примере, приведенном на рис. 4.5, - это отрезки 1-9, 5-4 и 201-203; общие результаты расчета расстояний между точками, а также их координат приведены в Приложении А (табл. А.2).

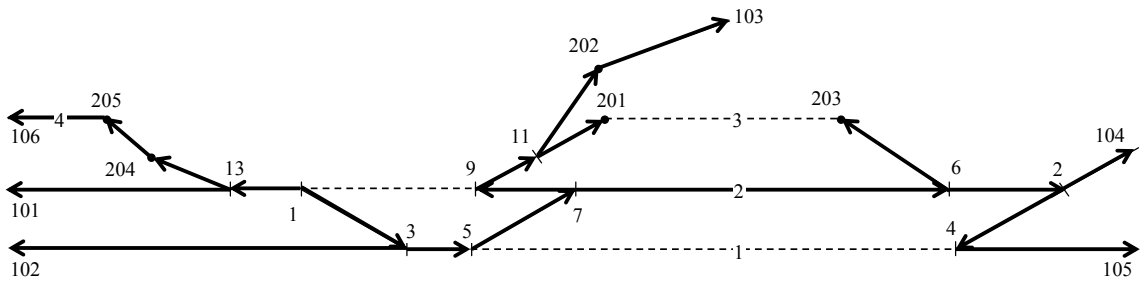

Рисунок 4.5 - Остовное дерево $U=(V, E)$ на графе $\bar{G}$ для расчета абсцисс $x_{i}$ точек плана

\section{4. Расчет сокращенных соединений}

Проектирование соединений параллельных путей при больших междупутьях осуществляют с укладкой дополнительных кривых, позволяющих 
уменьшить общую длину соединений (сокращенные соединения, съезды, улицы, а также горловины разнообразной конструкции). Основной задачей при проектировании сокращенных соединений является расчет углов поворота дополнительных кривых. В этой связи была разработана универсальная методика расчета любых сокращенных соединений с обратными кривыми; она основана на известном методе расчета сокращенных улиц [2]. Метод основан на вычислении сумм проекций элементов расчетного пути между вершинами обратных кривых на горизонтальные и вертикальные оси соответствующей системы координат.

Методику расчета неизвестных углов рассмотрим на примере стрелочной горловины, приведенной на рис. 4.6. В данной горловине для сокращения ее длины уложена дополнительная кривая 201 с углом поворота $\beta$ и обратные ей кривые 203-207, соответственно, на путях 3-7. Для определения значения угла $\beta$ должен быть выбран расчетный путь, соединяющий вершину 201 с вершиной одной из обратных кривых. Для примера рассмотрим расчетный путь между вершинами 201-207, схема которого приведена на рис. 4.7.

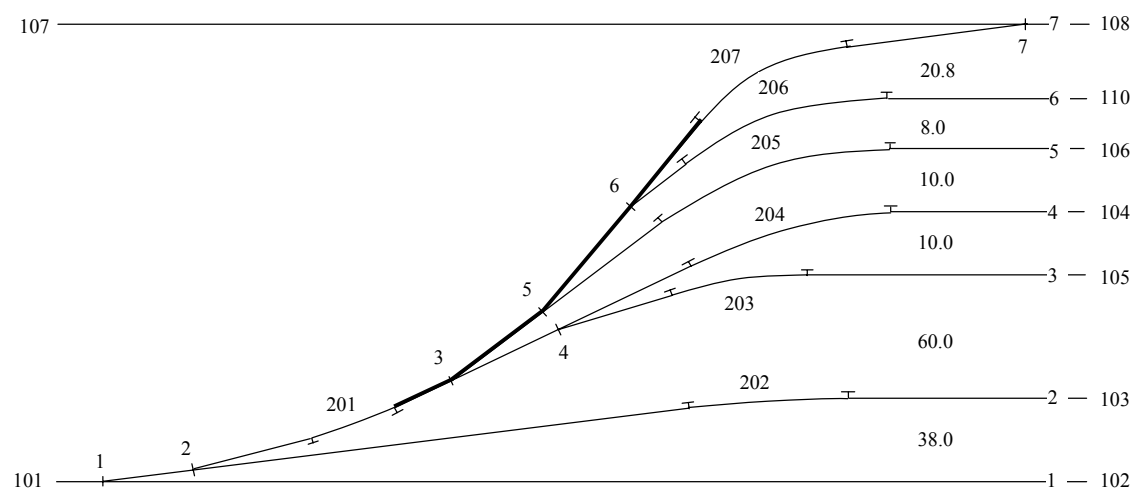

Рисунок 4.6 - Схема стрелочной горловины с обратными кривыми

На данном рисунке расчетный путь 201-207 построен в основной системе координат $X 0 Y$; кроме того, введена дополнительная система $x 0 y$, начало которой совпадает с концом кривой 201, а ось абсцисс проходит через отрезок 201-3 (отрезок, исходящий из вершины неизвестного угла). В дополни- 
тельной системе $x 0 y$, в отличие от основной $X 0 Y$, известны углы наклона элементов расчетного пути к осям координат. Это позволяет найти сумы проекций элементов расчетного пути на оси координат, которые необходимы для определения угла $\beta$.

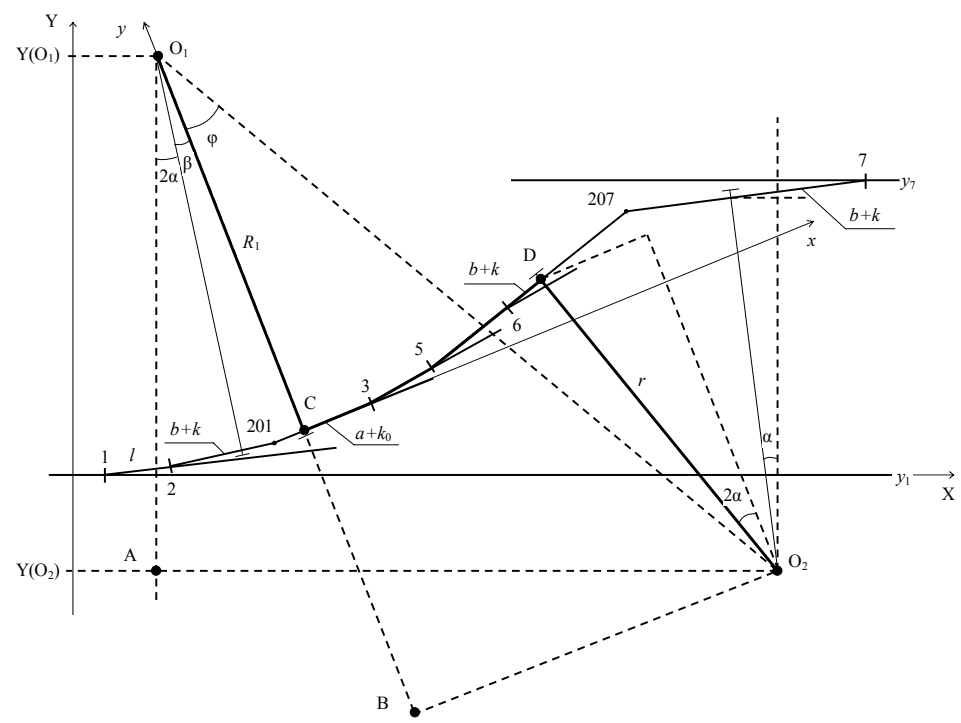

Рис. 4.7 Схема расчетного пути между вершинами 201-207

Неизвестный угол $\beta$ можно найти из $\Delta O_{1} A O_{2}$ (рис. 4.7):

$$
\beta=\arccos \frac{A O_{1}}{O_{1} O_{2}}-(2 \alpha+\varphi)
$$

Величину угла $\varphi$, в свою очередь, можно определить из $\triangle O_{1} B O_{2}$ :

$$
\varphi=\operatorname{arctg} \frac{B O_{2}}{B O_{1}}
$$

Стороны треугольника $B O_{1}$ и $B O_{2}$, очевидно, равны суммам проекций ломаной линии $O_{1}-C-3-5-7-D-O_{2}$, соответственно, на оси $0 y\left(S_{y}\right)$ и $0 x\left(S_{x}\right)$ :

$$
\begin{aligned}
& S_{y}=R-\sum_{1}^{n} l_{i} \sin \theta_{i}+r \cdot \cos \sum_{1}^{n} \theta_{i} \\
& S_{x}=\sum_{1}^{n} l_{i} \cos \theta_{i}+r \cdot \sin \sum_{1}^{n} \theta_{i},
\end{aligned}
$$


где $l_{i}, \theta_{i}-$ соответственно, длина и угол наклона к оси абсцисс элементов расчетного пути;

$n$ - число элементов;

$R, r$ - радиусы, соответственно, начальной и конечной кривых на расчетном пути.

В приведенных выражениях (4.8) значение $\theta_{i}$ принимают с учетом знака. Значение угла $\varphi$ (4.7) используется для расчета гипотенузы $O_{1} O_{2}$ в $\Delta O_{1} B O_{2}$, необходимой при определении угла $\beta(4.6)$ :

$$
O_{1} O_{2}=\frac{S_{y}}{\cos \varphi} .
$$

Катет $A O_{1}$ в (4.6) можно найти как разность ординат точек $O_{1}$ и $O_{2}$ в основной системе координат

$$
A O_{1}=Y\left(O_{1}\right)-Y\left(O_{2}\right) .
$$

Ординаты $Y\left(O_{1}\right)$ и $Y\left(O_{2}\right)$ находят по установленным ординатам горизонтальных путей, с которыми связаны, соответственно, точки $O_{1}$ и $O_{2}$ цепочками отрезков и углом наклона к оси $0 X$. Для рассматриваемого примера указанные ординаты определяются как

$$
\begin{aligned}
& Y\left(O_{1}\right)=Y_{1}+l \cdot \sin \alpha+(b+k) \sin 2 \alpha+R \cdot \cos 2 \alpha, \\
& Y\left(O_{2}\right)=Y_{7}-(b+k) \sin \alpha-r \cdot \cos \alpha .
\end{aligned}
$$

После расчета неизвестного угла $\beta$ остальные параметры горловины определяются обычным образом.

Для задания расчетного пути необходимо в канонической модели горловины в строке вершины 201 вместо неизвестного значения угла $\alpha$ указать номер конечной вершины расчетного пути; в рассматриваемом примере эта строка будет выглядеть как

\begin{tabular}{|l|l|l|l|l|l|l|l|l|}
\hline $\mathrm{N}$ & $\mathrm{N}_{P}$ & $\mathrm{~N}_{B}$ & $\mathrm{~W}$ & $\mathrm{R}$ & $\mathrm{L}_{P}$ & $\alpha^{\circ}$ & $\alpha^{\prime}$ & $\alpha^{\prime \prime}$ \\
\hline 201 & 3 & 0 & 0 & 200 & 0 & 207 & & \\
\hline
\end{tabular}


Общий вид модели горловины, приведенной на рис. 4.6, и результаты ее расчета приведены в Приложении В.

\section{5. Расчет специализированных стрелочных улиц}

Конструкция стрелочных улиц определяется схемой размещения отдельных стрелочных переводов и углом наклона улицы к оси основного пути. Основными типами стрелочных улиц являются простейшие (под углом крестовины и на основном пути), комбинированные, сокращенные, улицы под двойным углом крестовины, веерные и пучкообразные.

При расчете стрелочных улиц обычно известны расстояния $е$ между осями параллельных путей, радиусы $R$ сопрягающих кривих, параметры стрелочных переводов, а также нормативные данные о вставках между отдельными элементами улицы (стрелочными переводами, сопрягающими кривыми). Расчет простейших и комбинированных улиц обычно не вызывает трудностей и может выполняться по общей методике, изложенной выше.

Пучкообразные стрелочные улицы из симметричных стрелочных переводов применяют в горочных горловинах сортировочных парков. Они обычно проектируются в комплексе с другими элементами плана горочных горловин; их расчет рассмотрен в п. 5.2. При проектировании других типов улиц (сокращенных, под двойным углом крестовины и веерных) используются специализированные методы расчета, которые рассматриваются ниже.

\subsection{1. Сокращенные стрелочные улицьь}

Стрелочная улица данного типа имеет увеличенный наклон (угол наклона $\beta>\alpha$ ), образующийся за счет укладки дополнительной кривой после первого стрелочного перевода (см. рис. 4.8). Это позволяет сделать ее более короткой, по сравнению с простейшей улицей, и сократить длину маневровых рейсов.

Основными расчетными параметрами сокращенных улиц являются угол наклона $\beta$, величина которого зависит от расстояний $\left(e_{1}, e_{2}, \ldots, e_{n-1}\right)$ меж- 
ду примыкающими путями, а также вставки $f_{i}$ между стрелочными переводами и кривыми.

Величина угла $\beta$, как известно [2], зависит от минимального междупутья $e_{\min }=\min \left(e_{2}, \ldots, e_{n-2}\right)$.

$$
\beta=\arcsin \frac{e_{\min }}{a+d+b} .
$$

Здесь вставка $d$ принимается в соответствии со схемой 3 попутной укладки (см. п. 4.2).

При междупутьях $e_{i}>e_{\min }$ целесообразно увеличивать вставку между соответствующими стрелочными переводами на улице для того, чтобы не увеличивать вставки на примыкающих путях и не сокращать их прямолинейную часть:

$$
f_{i}=\frac{e_{i}}{\sin \beta}-(a+b)
$$

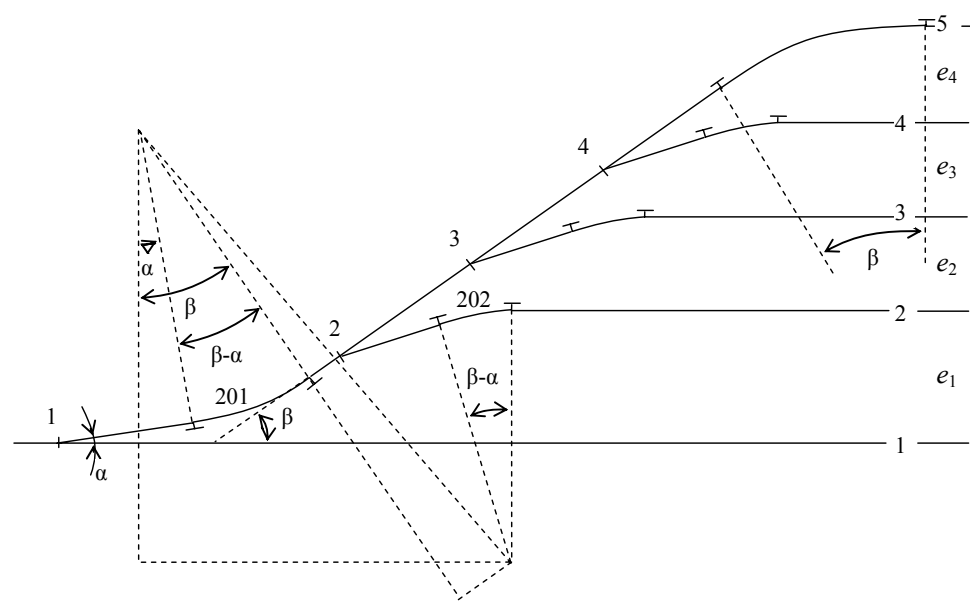

Рисунок 4.8 - Сокращенная стрелочная улица

При рассчитанном значении угла $\beta$ (4.9) необходимая величина первого междупутья $e_{1 \mathrm{p}}$ (см. рис. $\left.4.9, a\right)$ определяется из условия обеспечения минимальных величин вставок $k$ на основном пути ([1], табл. 16.51) и $\max \left\{k, k_{0}\right\}$ на боковом пути ([1], табл. 16.52). 
Для приведенного примера при $e_{\min }=5,3$ м указанные параметры соответственно равны $\beta=1^{\circ} 49$ '34" и $e_{1_{\mathrm{p}}}=7,611$ м; результаты расчета приведены в Приложении Г (табл. Г.1).

a)
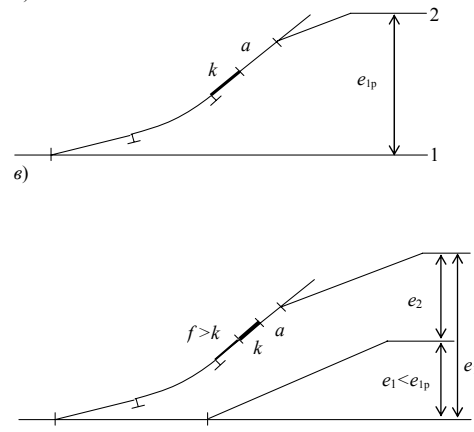

б)

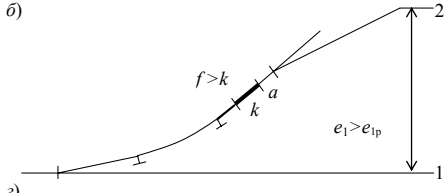

2)

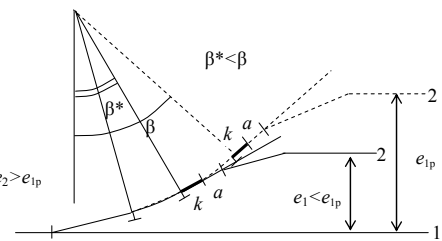

Рисунок 4.9 - Выбор первого междупутья в сокращенных улицах

Следует заметить, однако, что потребная величина первого междупутья $e_{1}$, как правило, не совпадает с расчетным значением $e_{1 \mathrm{p}}$. Если при этом первое междупутье достаточно велико $\left(e_{1}>e_{1 \mathrm{p}}\right)$, то в этом случае необходимо сместить стрелочный перевод второго пути вдоль улицы, увеличив вставку 201-2 (см. рис. 4.9, б). Это позволит сохранить минимальную вставку 2-202 $\left(k_{0}\right)$ и за счет этого максимизировать прямолинейную часть пути 2.

Если же первое междупутье мало $\left(e_{1}<e_{1 \mathrm{p}}\right)$, то в этом случае возможны два решения. Первое заключается в том, чтобы переложить стрелочный перевод второго пути на основной путь 1 (см. рис. 4.9, в); в этом случае схема расчета станет такой же, как в первом случае при $e_{1}>e_{1 \mathrm{p}}$.

Второе решение состоит в том, чтобы уменьшить значение угла $\beta$ по сравнению с рассчитанным (4.9). В этом случае величина $\beta^{*}<\beta$ устанавливается такой, чтобы сумма проекций элементов расчетного пути 2 на вертикальную ось равнялась $e_{1}$ (см. рис. 4.9, 2).

При любом значении угла наклона улицы $\beta$ необходимо проверить величину вставки $f_{n}$ на последнем пути (см. рис. 4.10 ): 


$$
\frac{e_{n-1}+\left(b+f_{n-1}+T_{n-1}\right) \cdot \sin (\beta-\alpha)}{\sin \beta}-\left(b+T_{n}\right) \geq \max \left\{k, k_{0}\right\} .
$$

Если данное условие не выполняется, то необходимо либо увеличить междупутье $e_{n-1}$, либо уменьшить угол $\beta$, либо сократить вставки между стрелочными переводами улицы, чтобы сместить стрелку и увеличить вставки $f_{n-1}, f_{n}$.

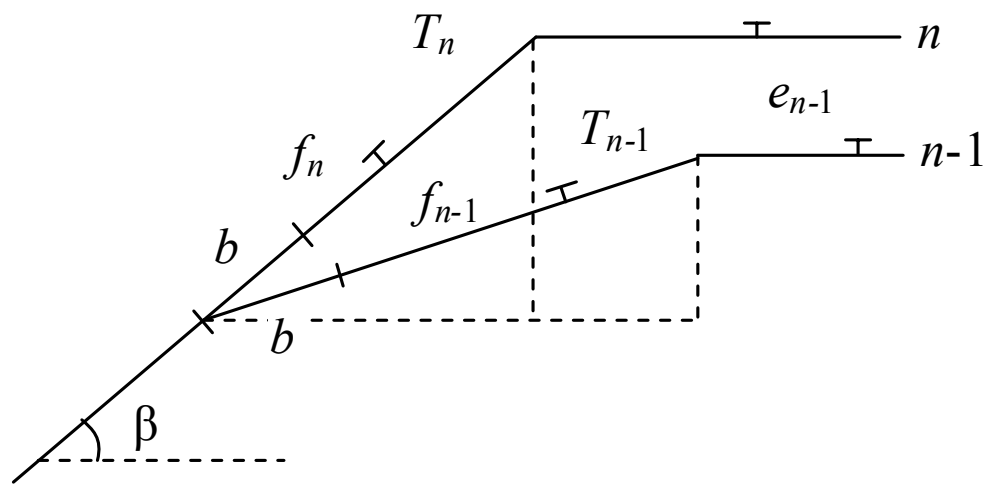

Рисунок 4.10 - Схема расчета вставки на последнем пути сокращенной улицы

Ниже рассмотрены особенности канонической модели для расчета сокращенных улиц при различных соотношениях между их параметрами.

Если первое междупутье $e_{1}$ превышает расчетное значение $e_{1 \mathrm{p}}$, то в данном случае величина угла наклона улицы $\beta$ ограничена сверху и определяется минимальным расстоянием между осями смежных путей $e_{\min }$ и принятой конструктивной вставкой между стрелочными переводами улицы, ведущими на эти пути (4.9). Следовательно, при этом угол $\beta$ нельзя найти по элементам расчетного пути 1-201-2-202, поскольку один из них (201-2) неизвестен. Поэтому для автоматизированного расчета такой стрелочной улицы необходимо в ее канонической модели указать в явном виде величину вставки 201-2 и номер второй вершины (202), определяющей расчетный путь 1-201-2-202, по элементам которого и будет определено значение угла $\beta$. Ниже приведена соответствующая строка модели улицы: 


\begin{tabular}{|l|l|l|l|l|l|l|}
\hline $\mathrm{N}$ & $\mathrm{P}$ & $\mathrm{B}$ & $\mathrm{W}$ & $\mathrm{R}$ & $\mathrm{L}_{P}$ & $\mathrm{~A}$ \\
\hline 201 & 2 & 0 & 0 & 200 & 24.8145 & 202 \\
\hline
\end{tabular}

При этом вставки между стрелочными переводами улицы (2-3 и 3-4) могут быть заданы явно $\left(L_{P}=6.25\right.$ м), однако лучше, чтобы они были рассчитаны автоматически по результатам вычисления угла $\beta$, т.е. должно быть задано $L_{p}=9999$. Последний подход особенно удобен, если междупутья $e_{2}, \ldots$, $e_{n-2}$ различны, и, соответственно, вставки на улице также должны быть разными; поэтому данные о стрелках, расположенных на улице, представляются как $\left(\mathrm{L}_{P}=9999\right)$

\begin{tabular}{|l|l|l|l|l|l|l|l|}
\hline $\mathrm{N}$ & $\mathrm{P}$ & $\mathrm{B}$ & $\mathrm{W}$ & $\mathrm{S}$ & $\mathrm{T}$ & $\mathrm{L}_{P}$ & $\mathrm{~L}_{B}$ \\
\hline 2 & 3 & 202 & 0 & 1 & 0 & 9999 & \\
\hline
\end{tabular}

Следует заметить, что отсутствие в данной строке значения $L_{P}$ будет воспринято как $L_{P}=0$; при этом вставка 2-3 будет отнесена к схеме 4, что приведет к ошибочному результату расчетов.

Другая возможность представления расчетного пути в модели состоит в том, чтобы указать в явном виде угол $\beta$ :

\begin{tabular}{|c|c|c|c|c|c|c|c|c|}
\hline $\mathrm{N}$ & $\mathrm{P}$ & $\mathrm{B}$ & $\mathrm{W}$ & $\mathrm{R}$ & $\mathrm{L}_{P}$ & $\alpha^{\mathrm{o}}$ & $\alpha^{\prime}$ & $\alpha^{\prime \prime}$ \\
\hline 201 & 2 & 0 & 0 & 200 & 9999 & 1 & 49 & 34 \\
\hline
\end{tabular}

При этом неизвестная вставка 201-2 должна быть обязательно заменена кодом 9999; в этом случае она будет рассчитана автоматически из условия обеспечения минимальной вставки 2-202 на боковом пути стрелки 2. Второй подход является предпочтительным, поскольку расчет угла $\beta$ (4.9) проще, чем вставки 201-2. Полный вид внутренней модели для расчета сокращенной стрелочной улицы при $e_{1}=10 \mathrm{~m}>e_{1 \mathrm{p}}$ и результаты автоматизированного расчета приведены в Приложении Г (табл. Г.2).

В случае, когда первое междупутье $e_{1}$ меньше расчетного значения $e_{1 \mathrm{p}}$, возможны два различных решения. Первое из них предполагает перенос 
стрелочного перевода для второго пути на основной путь; если при этом междупутье $e_{1}{ }^{\prime}=e_{1}+e_{2}$ превысит расчетное значение, то расчет улицы осуществляется так же, как было указано выше. Другое решение предусматривает уменьшение угла наклона улицы $\beta$ до величины, которая допускает укладку всех элементов расчетного пути 1-201-2-202. В этом случае в канонической модели улицы для вершины неизвестного угла $\beta$ необходимо указать конечную вершину расчетного пути (202), по элементам которого будет определено значение этого угла. Для определения вставки 201-2 необходимо задать $\mathrm{L}_{P}=0$; при этом в расчетах будет принято минимальное значение указанной вставки, равное $k$ :

\begin{tabular}{|l|l|l|l|l|l|l|}
\hline $\mathrm{N}$ & $\mathrm{P}$ & $\mathrm{B}$ & $\mathrm{W}$ & $\mathrm{R}$ & $\mathrm{L}_{P}$ & $\mathrm{~A}$ \\
\hline 201 & 2 & 0 & 0 & 200 & 0 & 202 \\
\hline
\end{tabular}

Следует заметить, что при уменьшении угла $\beta$ против расчетного (4.9), необходимо соответственно увеличить и вставки между стрелочными переводами улицы, чтобы не допустить сокращения длины горизонтальной части примыкающих путей; указанные вставки рассчитываются автоматически при задании в модели для соответствующих стрелок значений $\mathrm{L}_{P}=9999$. Модель для расчета сокращенной стрелочной улицы при $e_{1}=6.5$ м $<e_{1 \mathrm{p}}$ и его результаты приведены в Приложении Г (табл. Г.3).

При выполнении практических расчетов на первом этапе, пока неизвестно соотношение между заданной $e_{1}$ и расчетной $e_{1 \mathrm{p}}$ величинами первого междупутья, необходимо вычислить значение угла $\beta$ (4.9) и выполнить расчет улицы, используя форму представления модели (4.10). Дальнейшие действия зависят от результатов анализа рассчитанной величины вставки $f_{201-2}$. Если $f_{201-2}>k$, то это значит, что $e_{1}>e_{1 \mathrm{p}}$, и расчеты на этом заканчиваются; в противном случае необходимо найти уменьшенное значение угла $\beta$, при котором $f_{201-2}=k$. С этой целью необходимо повторить расчет улицы, заменив строку модели (4.10) на (4.11). 
Таким образом, разработанная геометрическая модель сокращенной улицы позволяет практически полностью автоматизировать ее расчет при любых возможных сочетаниях ее параметров.

\subsection{2. Стрелочная улица под двойным углом крестовинь}

Стрелочная улица под углом $2 \alpha$ позволяет сократить длину стрелочной зоны за счет увеличенного наклона к основному пути, уменьшает длину маневровых рейсов при перемещениях с одного пути на другой, а также обеспечивает лучшую видимость и удобное обслуживание удаленных стрелок (см. рис. $4.11, a$ ). Примыкающие пути, кроме последнего, объединяются в пары, так что число стрелочных переводов на улице сокращается вдвое. Первое междупутье приходится делать увеличенным для укладки головной стрелки улицы 2. В случаях, когда первое междупутье должно быть одинаковым с остальными, либо если в парке нечетное число путей, то конструкция улицы может быть несколько изменена за счет устройства примыкания второго пути к основному пути 1 (см. рис. $4.11, \sigma)$.

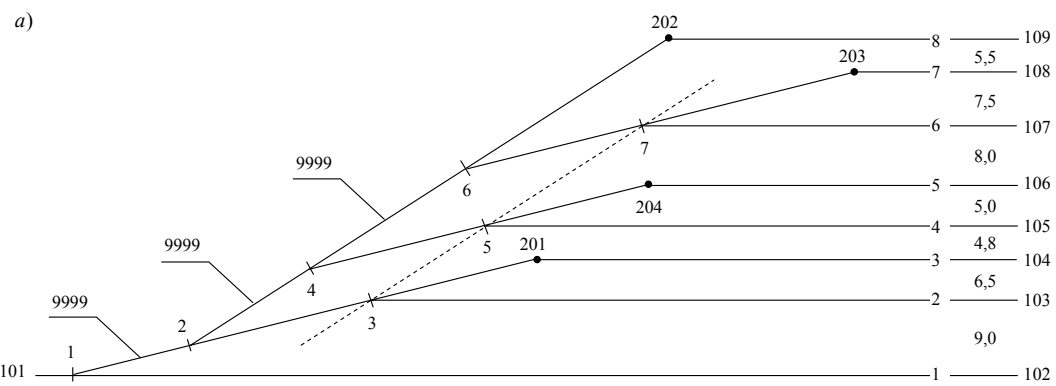

б)

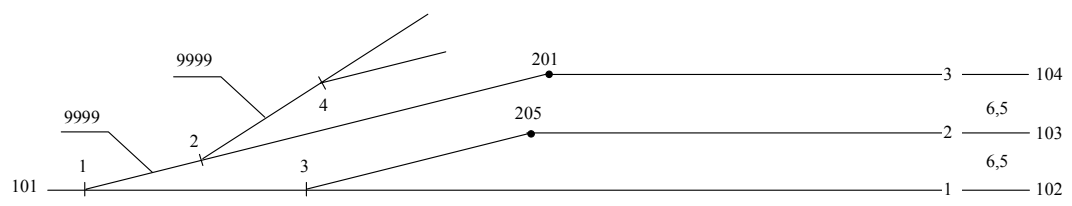

Рисунок 4.11 - Стрелочная улица под двойным углом крестовины

При расчете улиц известны расстояния $e_{i}, i=1,2, \ldots, n-1$ между осями путей, радиусы $R_{i}$ сопрягающих кривых, а также конструктивные вставки $d_{i}$, 
которые укладывают при соединении стрелочных переводов улицы и переводов, соединяющих пары примыкающих путей (см. рис. 4.11, $a$, отрезки 2-3, 4-5 и 6-7). Указанные пары стрелочных переводов укладывают по схеме 3 попутной укладки, так что вставки $d_{2-3}, d_{4-5}, d_{6-7}$ должны быть одинаковыми.

Расчету подлежат вставки улицы 2-4, 4-6, а также вставка 1-2; кроме того, как обычно, должны быть рассчитаны параметры сопрягающих кривых, а также координаты центров стрелочных переводов и вершин углов поворота кривых.

Вставки на стрелочной улице, как известно [2], рассчитываются по сумме двух смежных междупутий:

$$
f_{i}=\frac{e_{i}+e_{i+1}}{\sin 2 \alpha}-(a+b)
$$

Следует заметить, что стрелочные переводы улицы под углом $2 \alpha$ расположены по схеме 4 попутной укладки (см. п. 4.2), при которой расчет вставки $f_{i}$ осуществляется из условия обеспечения заданного междупутья между осями примыкающих боковых путей (4.3). В этой связи для правильного расчета вставок $f_{i}$ в модели данной улицы необходимо указать для них 9999. В этом случае расчет вставок осуществляется по разности ординат $y_{i}$ соответствующих стрелок, что соответствует (4.12):

$$
f_{i}=\frac{y_{i+1}-y_{i}}{\sin \theta},
$$

где $\quad \theta$ - угол наклона улицы к оси абсцисс.

При этом ординаты $Y_{i}, Y_{i+1}$ стрелок улицы определяют по известным ординатам $y_{i}$ смежных с ними переводов, находящихся на горизонтальных путях:

$$
Y_{i}=y_{i}-(a+d+b) \sin \alpha
$$

Первая вставка $f_{1,2}$ также должна быть помечена в модели кодом 9999; тогда ордината стрелки 2 будет вычислена по формуле (4.14), а вставка $f_{1,2}-$ по разности ординат $\Delta y=Y_{2}-Y_{1}$ (4.13).

В качестве опорной точки выбирают стрелочный перевод 1, находящийся на горизонтальном пути 1. 
Пример модели улицы под углом $2 \alpha$ и результаты ее автоматизированного расчета приведены в Приложении Г (табл. Г.4).

\subsection{3. Веерные стрелочные улиць}

Особенность веерных улиц заключается в том, что их ось представляет собой ломаную линию, направление которой изменяется на величину стрелочного угла $\alpha$ после примыкания каждого пути. Существует два типа веерных улиц: концентрические и неконцентрические.

Концентрические улицы имеют общий центр всех сопрягающих кривых; при этом радиус кривой для каждого последующего пути увеличивается на величину расстояния $e$ между осями данного и предыдущего путей (см. рис. 4.12). При такой конструкции улицы все точки сопряжения горизонтальных путей с кривыми имеют одинаковую абсциссу, а расстояния $L_{i}$ между центрами стрелочных переводов и соответствующие вставки $f_{i}$ увеличиваются по мере роста угла наклона элементов улицы.

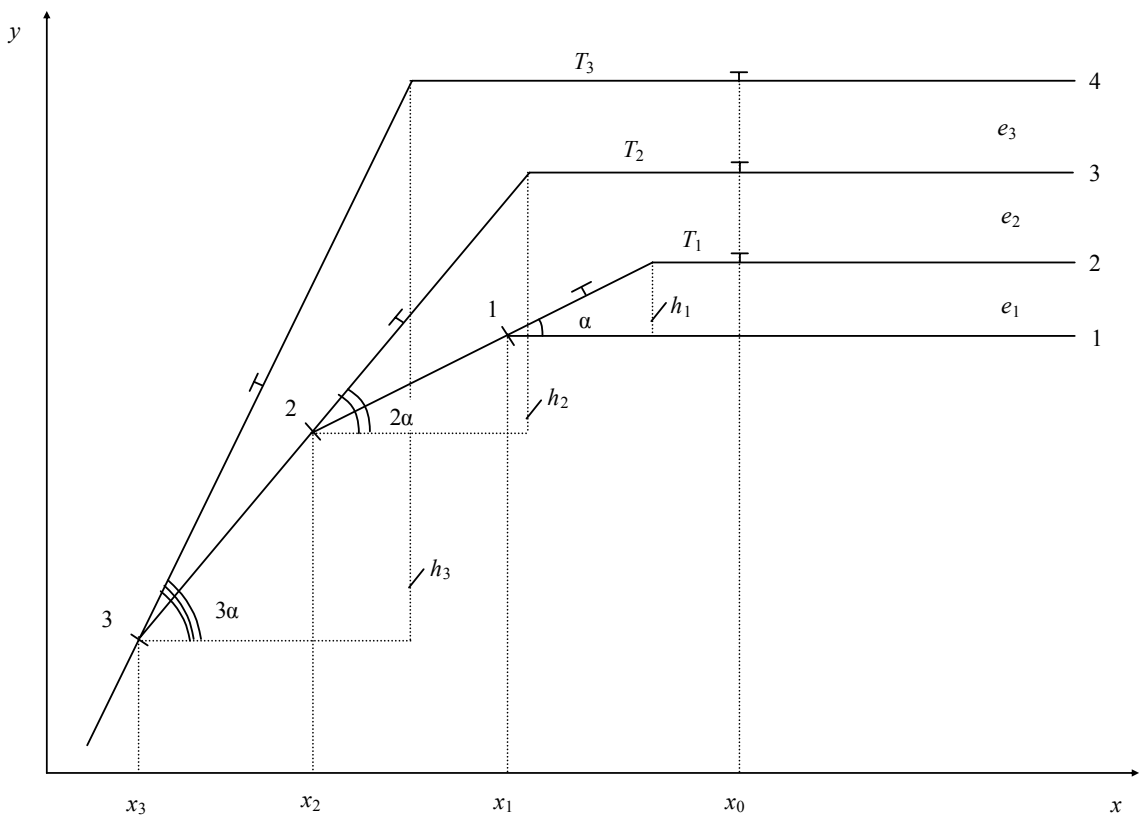

Рисунок 4.12 - Концентрическая стрелочная улица 
Неконцентрические улицы, напротив, имеют одинаковые расстояния $L_{i}$ между центрами переводов и соответствующие прямые вставки $d_{i}$, определяемые по схеме 3 попутной укладки (см. рис. 4.13). При этом радиусы сопрягающих кривых $R_{i}$ могут быть одинаковыми, либо увеличиваться на каждом последующем пути. Второй способ позволяет избежать уширения междупутий, которое имеет место при использовании радиусов постоянной величины, и тем самым сократить объем работ по сооружению улицы.

При расчете веерных стрелочных улиц обычно известны расстояния $e_{i}, i=1,2, \ldots, n-1$, между осями путей, радиусы сопрягающих кривых $R_{i}$, $i=1,2, \ldots, n$, а для неконцентрических улиц - и прямые вставки $d_{i}$ между стрелочными переводами. Рассчитываются параметры сопрягающих кривых, координаты центров переводов и вершин углов поворота, а для концентрических улиц - дополнительно определяются вставки $f_{i}$.

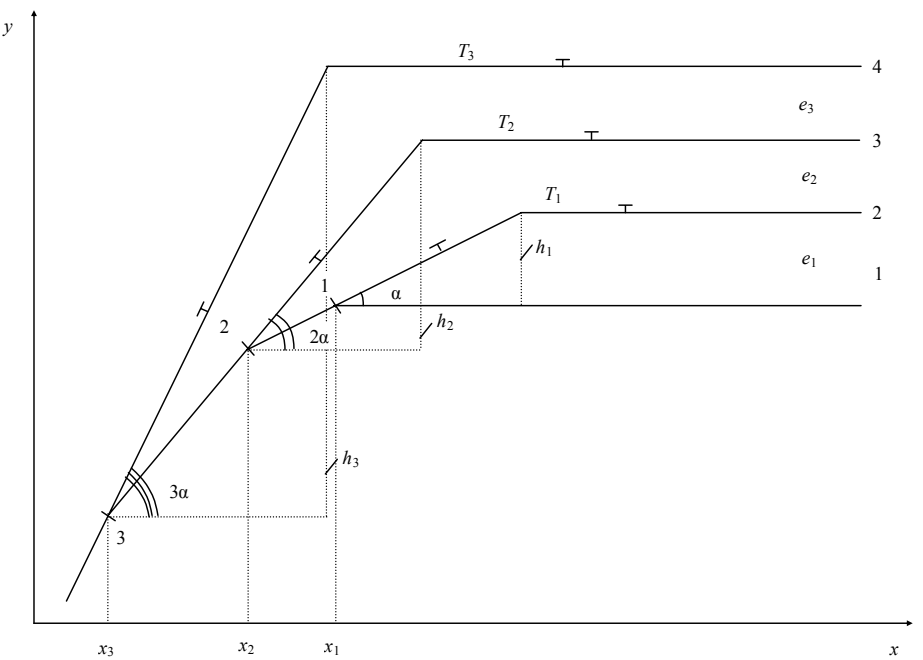

Рисунок 4.13 - Неконцентрическая стрелочная улица

Учитывая особенности конструкции концентрических улиц, отмеченные выше, вставки для них рассчитываются, исходя из условия равенства сумм проекций на ось абсцисс элементов двух смежных путей, начиная от центра их общего перевода и до точек сопряжения кривых с этими путями: 


$$
\begin{gathered}
T_{i}+\frac{h_{i}}{\operatorname{tg} i \alpha}+L_{i} \cdot \cos i \alpha=T_{i+1}+\frac{e_{i+1}+h_{i}+L_{i} \cdot \sin i \alpha}{\operatorname{tg}(i+1) \alpha}, \\
h_{i}=e_{i}+h_{i-1}+L_{i-1} \cdot \sin (i-1) \alpha, \\
T_{i}=R_{i} \cdot \operatorname{tg} \frac{i \alpha}{2}, \quad L_{i}=a+b+f_{i}, \\
i=1, \ldots, n-2, h_{0}=0, L_{0}=0 .
\end{gathered}
$$

Обозначения величин, входящих в данные выражения, понятны из рис. 4.12. В выражении (4.15) левая часть представляет собой проекцию $i$-го пути, а правая - $(i+1)$-го пути на ось $X$, которые, очевидно, должны быть равны. Величины $h_{i}$ определяются с помощью рекуррентного выражения (4.16); для первой пары путей $h_{1}=e_{1}$, поскольку $h_{0}=0$ и $L_{0}=0$. Как следует из выражений (4.15)-(4.17), вставки $f_{i}$ зависят от ширины междупутий $e_{i}$ и радиусов сопрягающих кривых $R_{i}$. Выполненные исследования показали, что в наибольшей степени вставка $f$ зависит от радиуса $R$. Для примера на рис. 4.14 показана зависимость $f=\varphi\left(R_{1}\right)$ величины $f$ от радиуса кривой на первом пути $R_{1}$. Как видно из рисунка, наименьшей является первая вставка $f_{1}$, которая при $R_{1}=$ 300 м составляет всего 2,17 м. При этом минимальный допустимый радиус, при котором $f_{1} \approx 0$, составляет $280 \mathrm{м}$; при увеличении радиуса $R_{1}$ величина $f_{1}$ существенно и практически линейно возрастает, так что при $R_{1}=400 \mathrm{м} f_{1}$ достигает 13,25 м. Из рис. 4.14 также видно, что вставки $f_{i}$ равномерно увеличиваются для каждого следующего пути, т.е. с ростом номера $i$.

Зависимость вставок $f_{i}$ от ширины междупутий $e$ показана на рис. 4.15. Оказалось, что первая вставка $f_{1}$ от междупутья $e$ не зависит; остальные вставки $f_{i}(i=2,3, \ldots)$ с увеличением $e$ линейно возрастают, причем эта зависимость тем сильнее, чем больше номер вставки $i$.

Характер изменения вставок $f_{i}$ по мере увеличения номера $i$ и их удаления от стрелки 1 при начальных радиусах $R_{1}=300$ м и $R_{1}=350$ м показан на рис. 4.16. Таким образом, выполненные исследования показывают, что минимальной является первая вставка $f_{1}$, величина которой зависит только от 
радиуса сопрягающей кривой $R_{1}$. Учитывая, что при попутной укладке стрелочных переводов по схеме 3 на приемо-отправочных путях величина вставки должна быть 6,25 м, а на прочих $-4,5$ м ([1,] табл. 16.7$)$, то радиус $R_{1}$ на первом пути должен быть, соответственно, 336,8 м и 321,0 м не зависимо от используемой ширины междупутий. В дальнейшем, по мере увеличения номера пути вставки $f_{i}$ будут возрастать, причем тем быстрее, чем больше ширина междупутий, так что требуемая минимальная величина вставки будет всегда обеспечиваться.

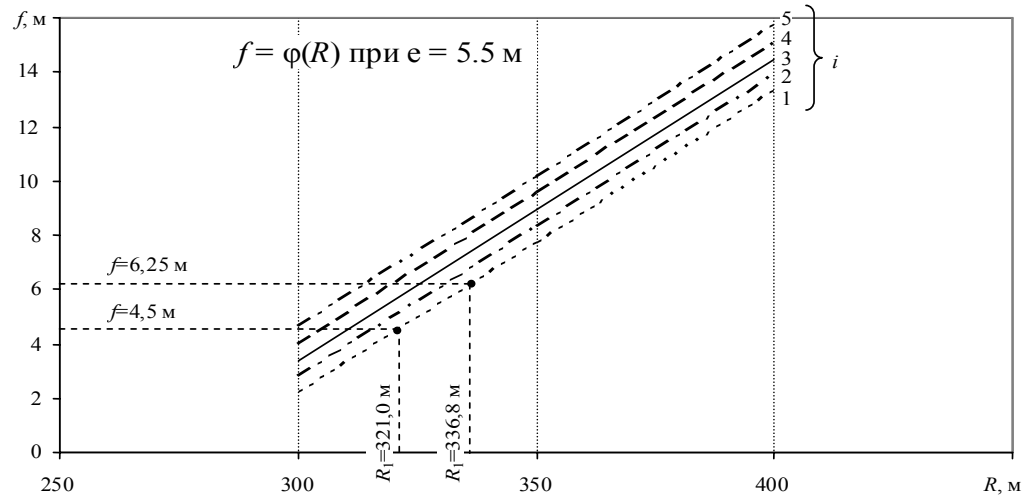

Рисунок 4.14 - Зависимости длин $f_{\text {i }}$ от радиуса $R_{1}$ для вставок $1-5$

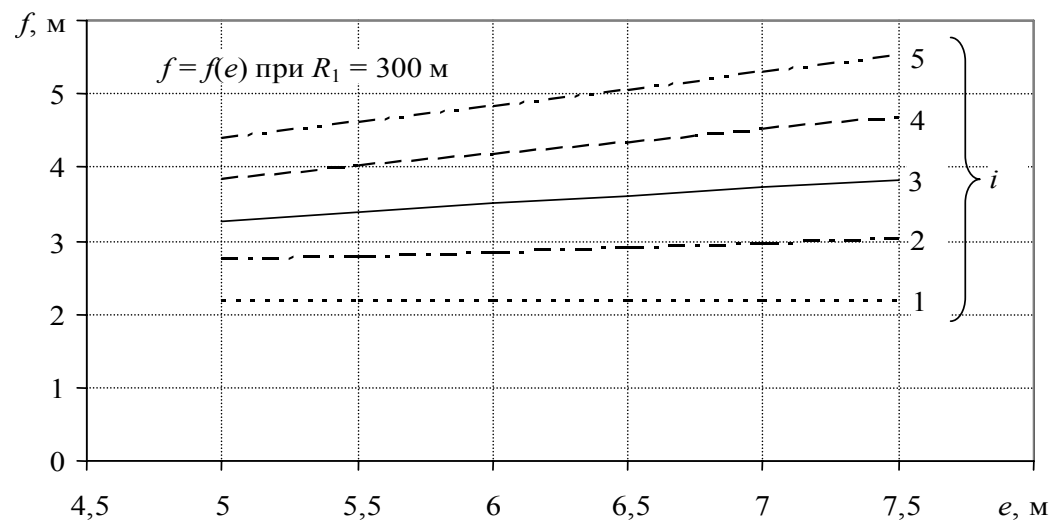

Рисунок -4.15 Зависимости длин $f_{\mathrm{i}}$ от ширины междупутий $e$ для вставок 1-5 


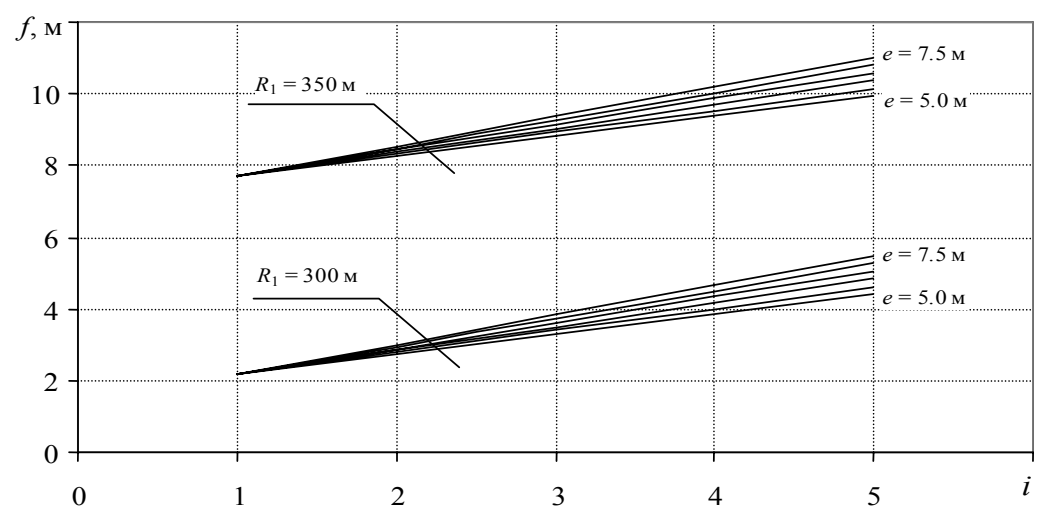

Рисунок 4.16 - Зависимости длин $f_{\mathrm{i}}$ от номера вставки $i$ при разных радиусах $R_{1}$ ширине междупутий $e$

Геометрические модели веерных улиц для их автоматизированного расчета формируют в соответствии с общими принципами (см. п. 2.2.1). При этом для концентрических улиц вставки должны быть рассчитаны по изложенной выше методике и включены в модель; для неконцентрических улиц все вставки определяются автоматически. В качестве опорной точки должен быть выбран стрелочный перевод 1, находящийся на горизонтальном пути.

Примеры автоматизированного расчета веерных стрелочных улиц приведены в Приложении Г (табл. Г.5, Г.6).

\section{6. Объединение базовых элементов в общий план станции}

Построение плана путевого развития сложных станций осуществляется путем объединения отдельных базовых элементов (блоков) в единую интегрированную модель в некоторой глобальной системе координат. Для включения очередного блока в общую совокупность необходимо преобразовать координаты его вершин из локальной системы координат в глобальную, а также выполнить сопряжения соответствующих путей.

Для выполнения преобразований координат удобно представить все характерные точки блока однородными координатами [29], которые точку $P(x, y)$ описывают трехэлементной вектор-строкой $\left[\begin{array}{lll}x & y & 1\end{array}\right]$. Тогда преобразо- 
вание координат точки $P$ осуществляется умножением вектора точки на матрицу преобразования $\mathbf{C}$ размером $3 \times 3$ :

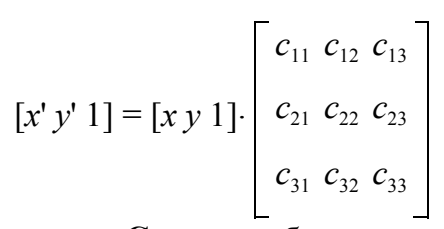

Матрица преобразования $\mathbf{C}$, может быть получена путем умножения матриц трех элементарных преобразований:

- перенос опорной точки $P_{0}\left(x_{0}, y_{0}\right)$ в начало координат локальной системы $\Sigma_{i}$;

- поворот локальной системы $\Sigma_{i}$ на угол $\varphi_{i}$;

- перенос начала координат локальной системы $\Sigma_{i}$ в точку $P_{0}\left(X_{0}, Y_{0}\right)$ глобальной системы $\Sigma$.

Указанная матрица преобразования может быть получена умножением матриц данных элементарных преобразований и имеет вид:

$$
\mathbf{C}=\left[\begin{array}{ccc}
\cos \varphi & \sin \varphi & 0 \\
-\sin \varphi & \cos \varphi & 0 \\
-\mathrm{x}_{0} \cos \varphi+\mathrm{y}_{0} \sin \varphi+X_{0} & -\mathrm{x}_{0} \sin \varphi-\mathrm{y}_{0} \cos \varphi+Y_{0} & 1
\end{array}\right]
$$

Структура последнего столбца матрицы позволяет упростить фактически выполняемые действия при преобразованиях координат:

$$
\begin{aligned}
& x^{\prime}=c_{11} x+c_{21} y+c_{31} \\
& y^{\prime}=c_{12} x+c_{22} y+c_{32}
\end{aligned}
$$

где $c_{i j}$ - коэффициенты результирующей матрицы преобразования $\mathbf{C}$, которые вычисляются однократно для всех точек блока;

$x, y$ - координаты опорной точки.

Расчет координат (4.18) выполняется для всех вершин блока; при этом координаты базовой вершины соответствуют координатам $X, Y$ точки размещения блока на общем плане станции (см. п. 2.4.2).

При проектировании плана путевого развития станции из отдельных базовых элементов, оси путей объединяемых блоков могут располагаться под 
углом друг к другу или на одной прямой. В первом случае необходимо выполнить сопряжение соответствующих путей, т.е. определить точки их пересечения (ВУП) и параметры сопрягающих кривых.

Для определения точки пересечения каждый из путей представляется параметрическим уравнением соответствующих прямых, проходящих через две точки (вершины) $a=\left(x_{a}, y_{a}\right)$ и $b=\left(x_{b}, y_{b}\right)$ этого пути:

$$
P(t)=a+t(b-a),
$$

где $t$ - параметр, который может принимать любое значение в диапазоне вещественных чисел (если значения параметра $t$ ограничены диапазоном $[0 ; 1]$, то уравнение (4.19) описывает отрезок прямой лини $\overline{a b}$ ).

Учитывая, что вершина КП имеет единственную входящую или исходящую дугу, необходимо на графе $G$ определить другую вершину, инцидентную этой дуге, и с ее помощью составить уравнение прямой (4.19).

С использованием теоремы о скалярном произведении векторов [28], получим выражение для определения параметра $t$, соответствующего точке пересечения $P(t)$ двух прямых линий $\overline{a b}$ и $\overline{c d}$ :

$$
t=\frac{\mathbf{q} \cdot(a-c)}{\mathbf{q} \cdot(b-a)},
$$

где $\mathbf{q}-$ вектор, перпендикулярный прямой $\overline{c d}$.

Если $\mathbf{q} \cdot(b-a)=0$, то вектор q перпендикулярен прямой $\overline{a b}$. Это свидетельствует о том, что прямые $\overline{a b}$ и $\overline{c d}$ либо параллельны, либо лежат на одной прямой, т.е. не имеют точки пересечения. В противном случае, когда прямые пересекаются, точку пересечения можно определить путем подстановки рассчитанного параметра $t$ в выражение (4.19).

При определении угла поворота сопрягающей кривой используется выражение:

$$
\varphi=\theta_{a v}-\theta_{v d}
$$

где $\theta_{a v}, \theta_{v d}-$ соответственно углы наклона отрезков $\overline{a v}$ и $\overline{v d}$ (см. рис. 4.17) 


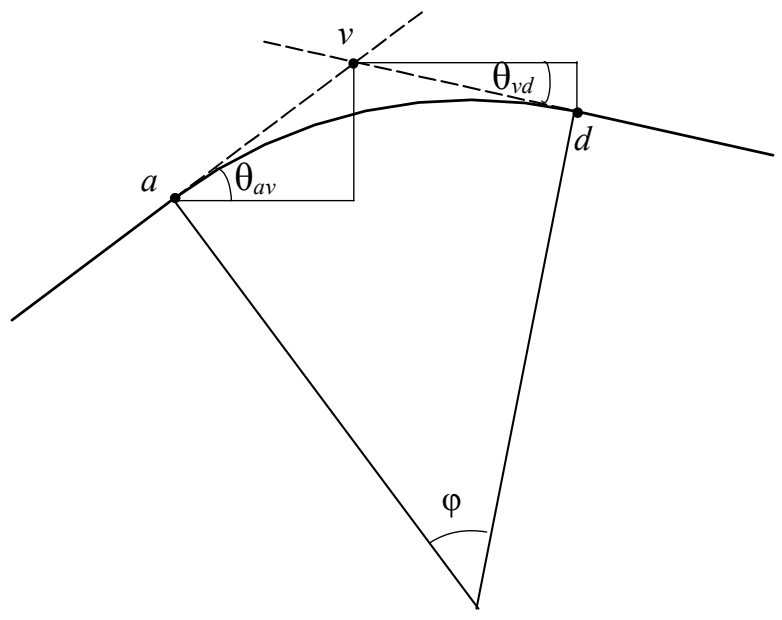

Рисунок 4.17 - Схема определения угла поворота кривой

В свою очередь угол наклона отрезка $\overline{a b}$ определялся как:

$$
\theta_{a b}=\arcsin \left(\frac{y_{b}-y_{a}}{\sqrt{\left(x_{b}-x_{a}\right)^{2}+\left(y_{b}-y_{a}\right)^{2}}}\right) .
$$

После определения угла поворота кривой рассчитываются остальные параметры - тангенс кривой $T$ и ее длина $K$.

При объединении блоков, пути которых лежат на одной прямой, угол поворота $\varphi=0$ и точки пересечения определять не нужно. В этом случае необходимо соединить соответствующие вершины дугой (см. рис. 4.18, a).

В результате объединения блоков формируется модель интегрированного блока, которая имеет такую же структуру (см. п. 2.4.2) и может быть использована для создания более сложных объектов. Созданному таким образом блоку устанавливается уникальный номер (имя) блока $N_{\text {б }}$, базовая вершина $v_{\sigma}$ и ориентированный граф $G$ с перенумерованными вершинами (см. рис. $4.18, \sigma)$. 
a)
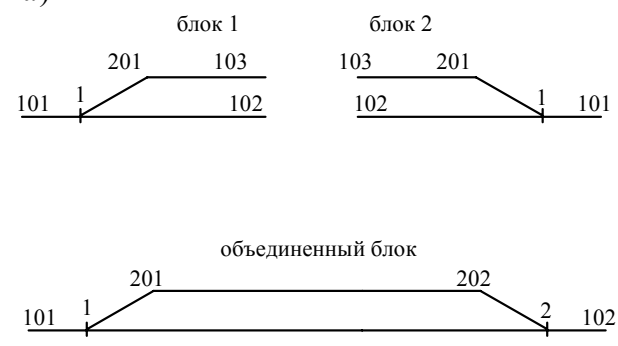

б)

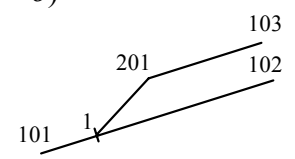

объединенный блок

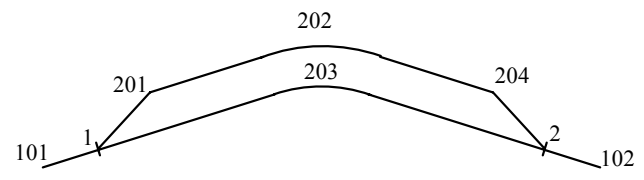

\begin{tabular}{ccc}
\multicolumn{3}{c}{ блок 1} \\
$N$ & $N_{\mathrm{P}}$ & $N_{\mathrm{B}}$ \\
\hline 1 & 102 & 201 \\
101 & 1 & 0 \\
102 & 0 & 0 \\
103 & 0 & 0 \\
201 & 103 & 0
\end{tabular}

\begin{tabular}{ccc}
\multicolumn{3}{c}{ блок 2} \\
$N$ & $N_{\mathrm{P}}$ & $N_{\mathrm{B}}$ \\
\hline 1 & 101 & 0 \\
101 & 0 & 0 \\
102 & 1 & 0 \\
103 & 201 & 0 \\
201 & 1 & 0
\end{tabular}

объединенный блок

\begin{tabular}{ccc}
$N$ & $N_{\mathrm{P}}$ & $N_{\mathrm{B}}$ \\
\hline 1 & 2 & 201 \\
2 & 102 & 0 \\
101 & 1 & 0 \\
102 & 0 & 0 \\
201 & 202 & 0 \\
202 & 1 & 2
\end{tabular}

\begin{tabular}{ccc}
\multicolumn{3}{c}{ блок 1} \\
$N$ & $N_{\mathrm{P}}$ & $N_{\mathrm{B}}$ \\
\hline 1 & 102 & 201 \\
101 & 1 & 0 \\
102 & 0 & 0 \\
103 & 0 & 0 \\
201 & 103 & 0
\end{tabular}

\begin{tabular}{ccc}
\multicolumn{3}{c}{ блок 2} \\
$N$ & $N_{\mathrm{P}}$ & $N_{\mathrm{B}}$ \\
\hline 1 & 101 & 0 \\
101 & 0 & 0 \\
102 & 1 & 0 \\
103 & 201 & 0 \\
201 & 1 & 0
\end{tabular}

объединенный блок

\begin{tabular}{ccc}
$N$ & $N_{\mathrm{P}}$ & $N_{\mathrm{B}}$ \\
\hline 1 & 2 & 201 \\
2 & 102 & 0 \\
101 & 1 & 0 \\
102 & 0 & 0 \\
201 & 202 & 0 \\
202 & 204 & 0 \\
203 & 1 & 0 \\
204 & 2 & 0
\end{tabular}

Рисунок 4.18 - Объединение блоков: $a$ - расположенных на прямой; $\sigma$ - расположенных под углом.

\section{7. Определение положения предельных столбиков и сигналов}

Методика расчетов построена на основе математической модели путевого развития в виде ориентированного графа $G=(V, E)$ (см. п. 2.2).

Анализ полустепеней исхода вершин, соответствующих стрелочным переводам $v \in V^{S}$, позволяет установить положение ПС относительно ЦП (см. рис. 4.19): $\operatorname{deg} v^{+}=1-$ ПС находится слева от ЦП; $\operatorname{deg} v^{+}=2-$ ПС находится справа от ЦП.

Как известно, ПС указывает границу, в пределах которой должен находится подвижной состав, не нарушая безопасности движения по соседнему пути. Если вагон, при прохождении ПС, остается на прямом участке, то ПС устанавливается на расстоянии $p=2,05$ м от оси пути. 
a)

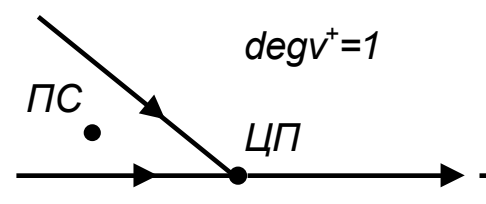

б)

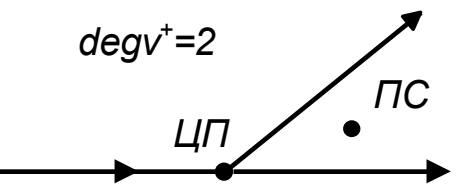

Рисунок 4.19 - Размещение предельного столбика относительно центра стрелочного перевода: $a-\operatorname{deg} v^{+}=1-$ ПС слева от ЦП; $\sigma-\operatorname{deg} v^{+}=2-$ ПС справа от ЦП

Если же при прохождении ПС вагон все время движется по круговой кривой, то расстояние $p$ должно быть увеличено на величину $\Delta_{\text {в }}$ или $\Delta_{\text {н }}$ (увеличение габаритного расстояния от оси пути до зданий и сооружений, находящихся, соответственно, с внутренней или наружной стороны кривой). В соответствии с [1] $\Delta_{\mathrm{B}}=l^{2} / 8 R, \Delta_{\mathrm{H}}=\left(L^{2}-l^{2}\right) / 8 R$; здесь $L-$ длина, а $l$ - база расчетного вагона $(L=24$ м,$l=17$ м), $R$ - радиус кривой. Следует заметить, что в научной и методической литературе недостаточно внимания уделено случаю установки ПС на участках перехода от прямой к кривой и обратно, где величины $\Delta_{\text {в }}$ и $\Delta_{\text {н }}$ имеют переменное значение. Между тем эти участки имеют длину порядка

30 м, так что в кривых малого радиуса ПС располагается именно на них. Таким образом, для автоматизации расчета координат ПС в этих случаях необходимо найти уравнения линий $h(x)$ и $g(x)$, описывающих наибольшее отклонение оси расчетного вагона от оси пути, соответственно, во внутреннюю и наружную стороны кривой.

Расчетная схема для идентификации функций $h(x)$ и $g(x)$ приведена на рис. 4.20. Здесь начало координат соответствует положению шкворня задней тележки вагона в момент, когда шкворень передней тележки находится в точке сопряжения прямой и кривой (точка $C$ ), т.е. начало координат находится на расстоянии $l$ от данной точки. Рассмотрим промежуточное положение вагона на участке перехода, которое характеризуется расстоянием $a$ между шкворнем задней тележки вагона $A$ и точкой $C$ (см. рис. 4.20). 


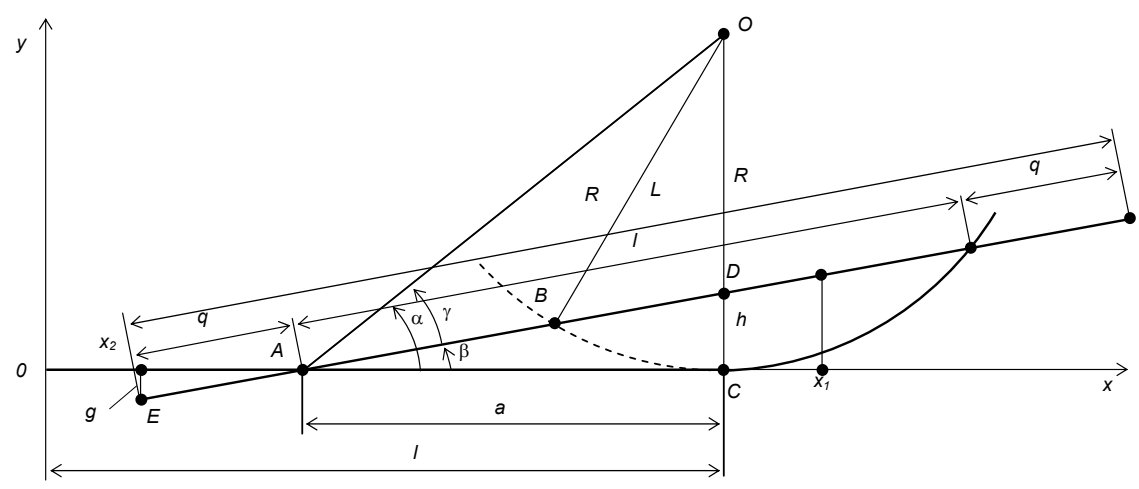

Рисунок 4.20 - Расчетная схема для определения смещения оси вагона относительно оси пути

В соответствии с приведенной схемой текущее значение угла поворота оси вагона $\beta$ относительно оси прямого пути определяется как:

$$
\beta=\alpha-\gamma
$$

Величину тангенса угла $\alpha$ можно найти из $\Delta \mathrm{ACO}$ :

$$
\operatorname{tg} \alpha=\frac{R}{a}
$$

С учетом (4.21) и (4.22) $\operatorname{tg} \beta$ можно определить с помощью выражения:

$$
\operatorname{tg} \beta=\frac{\operatorname{tg} \alpha-\operatorname{tg} \gamma}{1+\operatorname{tg} \alpha \cdot \operatorname{tg} \gamma}=\frac{R \cos \gamma-a \sin \gamma}{R \sin \gamma+a \cos \gamma}
$$

Величины $\cos \gamma$ и $\sin \gamma$ можно определяются из $\triangle \mathrm{ABO}$

$$
\begin{gathered}
\cos \gamma=\frac{a^{2}+l^{2}}{2 l \sqrt{a^{2}+R^{2}}} . \\
\sin \gamma=\sqrt{1-\cos ^{2} \gamma}=\frac{\sqrt{4 l^{2} R^{2}-\left(a^{2}-l^{2}\right)^{2}}}{2 l\left(a^{2}+R^{2}\right)^{2}}
\end{gathered}
$$

Тогда, подставляя выражения (4.24) и (4.25) в (4.23), получим:

$$
\operatorname{tg} \beta=\frac{R\left(a^{2}+l^{2}\right)-a \sqrt{4 l^{2} R^{2}-\left(a^{2}-l^{2}\right)^{2}}}{R \sqrt{4 l^{2} R^{2}-\left(a^{2}-l^{2}\right)^{2}}+a\left(a^{2}+l^{2}\right)}
$$

Ввиду малости величин $\left(a^{2}-l^{2}\right)^{2}$ и $a\left(a^{2}+l^{2}\right)$ по сравнению с $R^{2}$, выражение 
(4.26) упрощается и принимает вид:

$$
\operatorname{tg} \beta=\frac{(a-l)^{2}}{2 l R}
$$

Тогда в некоторой точке $x=x_{1}$ при заданном положении вагона $a$ величина смещения оси вагона от оси прямого пути равна:

$$
h(x, a)=(a+x-l) \operatorname{tg} \alpha=(a+x-l) \frac{(a-l)^{2}}{2 l R} .
$$

Очевидно, что величина смещения $h(x, a)$ в точке $x$ изменяется при изменении положения вагона $a$. Поэтому необходимо найти такое значение $a$, при котором функция (4.28) в точке $x$ достигнет максимума:

$$
h(x)=\max _{0 \leq a \leq l} h(x, a) .
$$

Используя классические методы оптимизации одной переменной, установлено, что $h(x, a)$ достигает максимума при $a=l-2 x / 3$ для любых значений $x$ в пределах $0 \leq x \leq x_{\max }$. Здесь $x_{\max }$ соответствует такому положению вагона, при котором шкворень его задней тележки (точка $A$ ) находится в точке сопряжения прямой и кривой (точка $C, a=0$ ) и равно

$$
x_{\max }=l\left(1+\frac{1}{2} \sqrt{1-\left(\frac{l}{2 R}\right)^{2}}\right) \approx 1,5 l .
$$

В точке $x_{\max }$ значение функции $h(x)$ достигает максимума $h\left(x_{\max }\right)=l^{2} / 4 R$; при этом увеличение габаритного расстояния принимает значение $\Delta_{\mathrm{B}}=l^{2} / 8 R$ и далее остается постоянным, пока обе тележки вагона находятся на кривой.

Таким образом, линия, описывающая наибольшее отклонение оси вагона от оси прямого пути во внутреннюю сторону кривой может быть представлена уравнением:

$$
h(x)=\frac{2 x^{3}}{27 l R}, \quad 0 \leq x \leq 1,5 l .
$$

Максимальное значение функции $g(x)$, описывающей отклонение во внешнюю сторону кривой, всегда соответствует конечной точке оси вагона (точка $E$, рис. 4.20 ) и равно $g=q \cdot \sin \beta$ (здесь $q=(L-l) / 2$ ). Ввиду малости угла $\beta$ 
$\left(\beta \leq 2,7^{\circ}\right) \sin \beta \approx \operatorname{tg} \beta$, а величина смещения, в соответствии с (4.27), равна:

$$
g \approx q \operatorname{tg} \beta=\frac{q(a-l)^{2}}{2 l R} .
$$

Следовательно, в некоторой точке $x=x_{2}$ функция $g(x)$ имеет вид:

$$
g(x)=\frac{q(x+q)^{2}}{2 l R} .
$$

Графическое изображение изменения смещения оси вагона относительно оси пути при движении на участке перехода от прямой к кривой приведено на рис. 4.21.

Окончательно выражения для определения значений функций $h(x)$ и $g(x)$ при произвольном значении $x$ приведены ниже.

$$
\begin{aligned}
& h(x)=\left\{\begin{array}{l}
0 \quad \text { при } x \leq 0 \\
\frac{2 x^{3}}{27 l R} \quad \text { при } 0<x<1,5 l \\
R-\sqrt{\left(R-\Delta_{\mathrm{B}}\right)^{2}-(x-l)^{2}} \text { при } x \geq 1,5 l
\end{array}\right. \\
& g(x)=\left\{\begin{array}{l}
0 \quad \text { при } x \leq-q \\
-\frac{q(x+q)^{2}}{2 l R} \quad \text { при }-q<x<l-q \\
R-\sqrt{\left(R+\Delta_{\mathrm{H}}\right)^{2}-(x-l)^{2}} \text { при } x \geq l-q
\end{array}\right.
\end{aligned}
$$

При изменении направления поворота кривой знаки выражений (4.29) и (4.30) изменяются на противоположные.

Для поиска координат ПС выполняется построение эквидистант кривых $h(x)$ и $g(x)$, их преобразование в систему координат станции, после чего определяются координаты точки их пересечения с использованием методов вычислительной геометрии. ПС централизованных стрелочных переводов устанавливаются на расстоянии 3,5 м от изолирующих стыков. В этой связи расстояние до ПС увеличивается и может быть определено с помощью выражения: 


$$
\left\{\begin{array}{l}
l_{\text {пс }}^{\prime}=\min \left(25 n_{25}+12,5 n_{12,5}+6,25 n_{6,25}+\right. \\
\left.+0,01\left(n_{25}+n_{12,5}+n_{6,25}-1\right)+b-3,5\right) \\
l_{\text {пс }}^{\prime} \geq l_{\text {пс }} ; \\
n_{25} \in[0, \infty] ; n_{12,5}=[0,1] ; n_{6,25}=[0,1]
\end{array}\right.
$$

где $l_{\text {пс }}-$ расстояние до ПС, рассчитанное из условия обеспечения габарита; $n_{25}, n_{12,5}, n_{6,25}$ - количество рубок рельсов, соответственно, длиной 25 , 12,5, и $6,25 \mathrm{M}$;

$b$ - расстояние от центра перевода до торца крестовины; 0,01 - стыковой зазор.

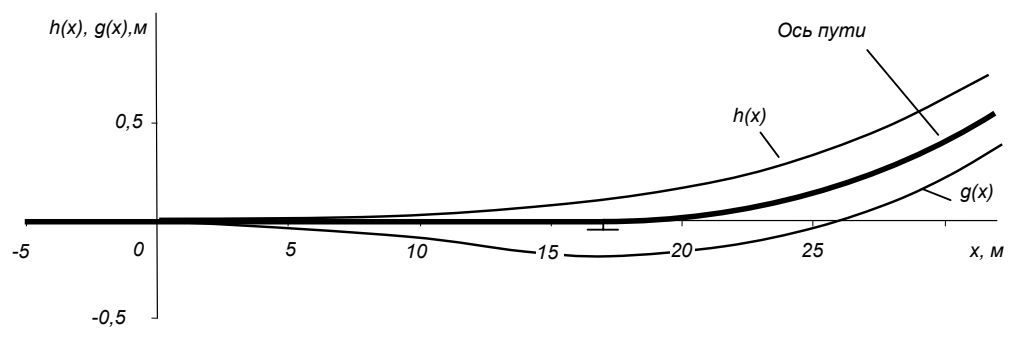

Рис. 4.21 Наибольшее смещение оси вагона относительно оси пути

Более сложной является задача определения положения сигналов. Эта задача решается в два этапа: определение схемы размещения сигналов относительно центров стрелочных переводов; расчет координат сигналов в соответствии с выбранной схемой.

Схема расположения сигнала $\sigma$ может быть однозначно установлена на основании параметра $z$ (см. п. 2.2.3) и направления $S$ (см. п. 2.2.3) стрелочного перевода $v$. При этом, если $z=2$, то сигнал устанавливается в створе изолирующего стыка рамного рельса ( $\sigma=2$, см. рис. $4.22, a, \sigma)$. При размещении сигнала в одном или разных междупутьях с ПС (см. рис. 4.22, в-e) схема расположения $\sigma$ устанавливается из выражения $\sigma=s \sim z$ ( - логическая операция эквивалентности). 


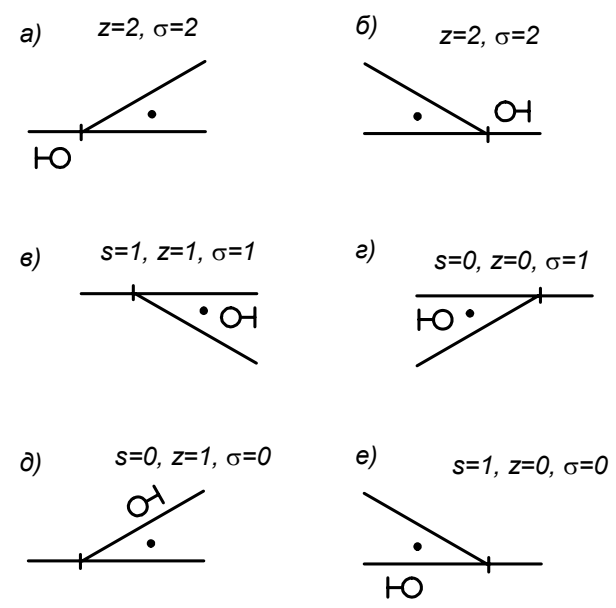

Рисунок 4.22 - Определение схемы расположения сигнала относительно центра стрелочного перевода: $a, \sigma$ - в створе изолирующего стыка рамного рельса $(\sigma=2) ;$;, 2 - на расстоянии $l_{\text {св }}$ от центра стрелочного перевода $(\sigma=1)$; $\partial, e-$ на расстоянии 3,5 м от ПС $(\sigma=0)$

Далее рассчитываются координаты установки сигналов с использованием методов вычислительной геометрии. При этом, расстояние от ЦП до сигнала определяется в соответствии со схемой б: при установке сигнала в створе изолирующего стыка рамного рельса $(\sigma=2) l_{\text {св }}=a$; при установке сигнала в разных междупутьях с ПС $(\sigma=0) l_{\mathrm{cB}}=l_{\text {пс }}+3,5$; при установке сигнала в одном междупутье с ПС $(\sigma=1)$ расстояние $l_{\text {св }}$ определяется из условия обеспечения габарита по методике, приведенной выше для ПС.

Результаты расчета координат предельных столбиков и сигналов используются при построении плана станции.

\section{8. Расчет потребной полезной длины путей на станциях}

\subsection{1. Сущуность задачи и методика ее решения}

При проектировании станций необходимо обеспечить потребную полезную длину всех станционных путей. Формально данное требование можно выразить условием

$$
L_{\text {фпд } i} \geq L_{\text {ппд } i}, \quad i=1, \ldots, n,
$$


где $\quad L_{\text {фпд } i}, L_{\text {ппд } i}-$ соответственно, фактическая и потребная полезные длины i-го пути;

$n$ - число путей станции.

При этом, учитывая особенности конструкции горловин станций, условие (4.31) для части путей будет являться равенством $\left(L_{\text {фпд } i}=L_{\text {ппд } i}\right)$, а для боль-

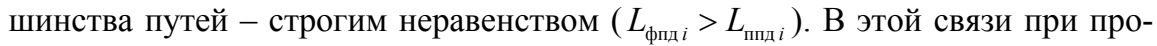
ектировании станций необходимо выделить пути с минимальной полезной длиной, для которых выполняется равенство $L_{\text {фпд } i}=L_{\text {ппд } i}$, обеспечить им потребную полезную длину, после чего определить полезные длины остальных путей.

Сложность данной задачи заключается в том, что путей с минимальной полезной длиной на станции может быть несколько (например, при наличии нескольких парков). Кроме того, правильный выбор пути с минимальной полезной длиной осуществить по немасштабной схеме станции без выполнения расчетов координат ее элементов достаточно сложно; в то же время для выполнения указанных расчетов необходимо предварительно установить потребные длины путей.

Автоматизированное решение этой противоречивой задачи для отдельного парка станции выполняется в три этапа. Первоначально определяются координаты точек обеих горловин парка при заданной длине одного из его путей (расчетного пути); при этом выбор пути и его длины может быть произвольным.

На втором этапе определяется потребное изменение длины расчетного пути, при котом все пути парка будут иметь полезную длину не менее заданной. На заключительном третьем этапе осуществляется повторный расчет координат всех точек парка при уточненной длине расчетного пути и определяются фактические полезные длины всех его путей.

Для сложных станций, состоящих из нескольких групп путей (парков) с различными потребными полезными длинами, расчет выполняется последо- 
вательно для каждой группы путей, которые выделяются автоматически в процессе построения остовного дерева на графе схемы станции.

\subsection{2. Методика расчета полезных длин путей в отдельном парке}

Фактические полезные длины путей парка определяются при известных координатах точек обеих его горловин; при этом для автоматизации расчетов необходимо разработать методику решения следующих задач:

- поиск на ориентированном графе станции дуги, являющейся частью пути, для которого определяется полезная длина (в дальнейшем - пути);

- поиск вершин, определяющих полную длину пути;

- поиск на полной длине пути точек, ограничивающих его полезную длину в заданном направлении;

- определение численного значения полезной длины пути.

Идентификация дуг графа, являющихся путями, осуществляется на основе анализа параметра $\mu$ (см. п. 2.1), который задает метод расчета длины каждой дуги. Путями являются дуги, у которых данный параметр имеет значение 4, 5 или 6, что означает необходимость расчета полезной длины в соответствующем направлении. Так, в результате поиска на графе, представленном на рис. 4.23, дуги $e_{1}, e_{2}$ и $e_{5}$ будут классифицированы как станционные пути.
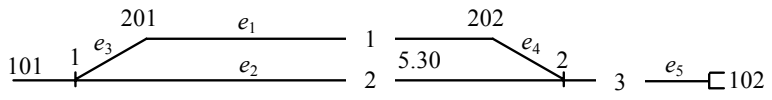

\begin{tabular}{|c|c|}
\hline № пути & дуги \\
\hline 1 & $\begin{array}{l}e_{3}=(1,201) \\
e_{1}=(201,202) \\
e_{4}=(202,2)\end{array}$ \\
\hline 2 & $e_{2}=(1,2)$ \\
\hline 3 & $e_{5}=(2,102)$ \\
\hline
\end{tabular}

Рисунок 4.23 - Представление путей на ориентированном графе

После определения дуги $e_{\text {п }}$, являющейся станционным путем или его частью, необходимо найти две вершины графа, ограничивающие его полную длину. Данными вершинами могут быть только центры стрелочных переводов и концы путей - упоры; поиск данных вершин производится в обе сторо- 
ны от найденной дуги $e_{п}$. Так, на графе, представленном на рис. 4.23, для дуги $e_{2}$ поиск не производится, поскольку ее начальная и конечная вершины являются ЦП; для дуги $e_{1}$ будут найдены связанные с ней дуги $e_{3}$ и $e_{4}$ и, соответственно, начальная (ЦП1) и конечная (ЦП2) вершины, определяющие полную длину пути 2.

Численное значение полной длины пути определяется суммированием длин элементов (отрезков, кривых), которые соответствуют входящим в нее дугам.

Полезная длина станционного пути может ограничиваться сигналом, предельным столбиком, упором или стыком рамного рельса стрелочного перевода. Если границей полной длины пути является упор, то координаты соответствующей вершины графа ограничивают и полезную длину этого пути. В случае, если границей полной длины пути является ЦП $\left(v_{i} \in V^{S}\right)$, для определения вершины, ограничивающей его полезную длину, необходимо предварительно установить направление $z_{i}$ входа этого пути в стрелочный перевод $v_{i}$ (см. рис. 4.22). Далее в списке сигналов $\mathbf{Q}$ (см. п. 2.2.3) производится поиск сигнала $q$, для которого выполняются условия:

$$
\left.\begin{array}{l}
v_{q}=v_{i} \\
z_{q}=z_{i}
\end{array}\right\}
$$

Если такой сигнал найден, то полезная длина пути определяется положением данного сигнала; в противном случае при $z=[0,1]$ полезная длина будет ограничиваться ПС, а при $z=2$ - изолированным стыком рамного рельса стрелочного перевода $v_{i}$.

Окончательно фактическая полезная длина $L_{\text {фпд }}$ станционного пути рассчитывается по формуле:

$$
L_{\text {фпд }}=L_{\text {полн }}-l_{\text {л }}-l_{\text {п }},
$$

где $L_{\text {полн }}-$ полная длина пути;

$l_{\text {л}}, l_{\text {п }}$ - расстояния между точками, ограничивающими полную и полезную длины путей, соответственно, слева и справа.

Значения величин $l_{л}, l_{\text {п }}$ в зависимости от параметра $z$ могут быть равны элементу $a$ стрелочного перевода, либо расстоянию $l_{\text {пс }}$ от ЦП до ПС (рас- 


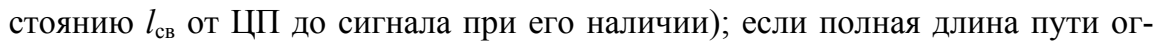
раничивается упором, то соответствующее значение $l_{\text {л }}$ или $l_{\text {п }}$ равно нулю.

\subsection{3. Алгоритм комплексного расчета полезных длин путей станциии}

Определение потребных полезных длины путей станции производится в комплексе с расчетом координат точек плана; решение данной задачи осуществляется в следующей последовательности (см. п. 4.8.1). На базе ориентированного графа станции $G=(V, E)$ строится остовное дерево $U=(V, E U)$ с корнем в вершине $v_{0}$, являющейся опорной точкой плана (см. п. 4.3). В дерево $U$ включаются те вершины, дуги между которыми представляют отрезки с известными длинами, определенными в процессе расчета плана станции; при этом дуги, соответствующие путям заданной длины, в дерево $U$ не включаются. Далее, начиная с опорной точки, выполняется расчет координат точек плана, соответствующих вершинам остовного дерева. Так, на графе станции, представленном на рис. 4.24, $а$ будет построено остовное дерево с корнем в вершине 1 и определены координаты точек 101, 2 и 201. (см. рис. 4.24, б).

Далее среди вершин построенного остовного дерева, выделяется вершина, исходящая дуга которой является путем с заданной во входной модели

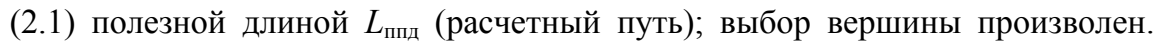
Так, например, на графе рис. 4.24, б выделена вершина 1 с исходящей дугой 1-4 (расчетный путь 1), которой во входной модели поставлена в соответствие полезная длина $L_{\text {ппд }}$

Выбранный расчетный путь представляет на схеме станции определенную группу путей, в которую он входит; при этом изменение длины расчетного пути ведет к такому же изменению длин остальных путей группы. Для обеспечения необходимых полезных длин путей указанной группы в построенное остовное дерево включается дуга, соответствующая выбранному пути. При этом для данного пути устанавливается некоторая достаточно большая

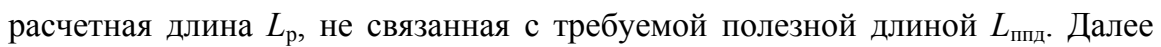
продолжается построение остовного дерева, в которое включаются все новые 
дуги исходного графа с известными длинами, связанные с дугой расчетного пути. Таким образом на первом этапе будет построено дерево, которое включает все вершины обеих горловин парка станции (в рассматриваемом примере на рис. 4.24, в - вершины 1-5 и 201-203).

После построения дерева в завершение первого этапа определяются координаты точек, соответствующих всем вновь включенным вершинам (вершины - 4, 203, 5, 3, 202).

На втором этапе по установленным координатам точек плана станции производится поиск на графе дуг, являющихся путями (дуги 1-4, 2-3 и 2-201-202-3), и определяются полезные длины соответствующих путей (пути $1-3$ ) в соответствии с методикой, изложенной в п. 4.8.2.

Далее, для каждого из путей группы производится расчет отклонения $\Delta L$ фактической полезной длины от потребной $\Delta L_{i}=L_{\text {фпді }}-L_{\text {ппдi }}$ Очевидно, что максимальное отклонение $\Delta L_{\max }=\max \left\{\Delta L_{1}, \ldots, \Delta L_{n}\right\}$ определяет необходимое изменение длины расчетного пути, обеспечивающее потребную полезную длину всех путей группы.
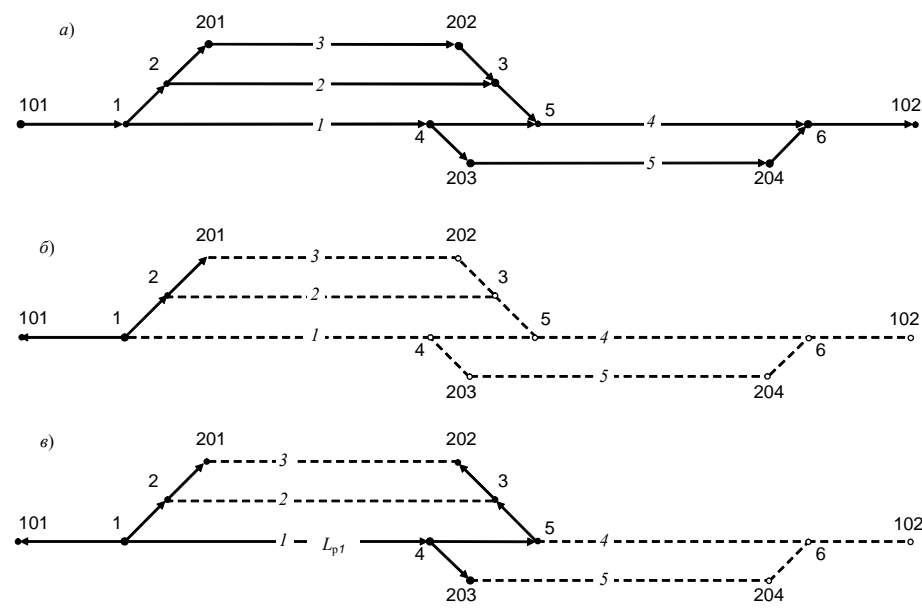

Рисунок 4.24 - Последовательность расчета полезных длин путей: $a-$ исходный граф станции; $\sigma$ - начало построения остовного дерева; 6 - остовное дерево для расчета длин группы путей 1-3 
На третьем этапе после увеличения длины расчетного пути на величину $\Delta L_{\max }$ осуществляется расчет окончательных значений координат точек плана станции, соответствующих вершинам остовного дерева, и фактических полезных длин путей группы.

В дальнейшем на графе станции осуществляется поиск новых вершин, включенных в построенное остовное дерево, с исходящими дугами, которые является путями, не включенными в дерево. Такими на графе рис. 4.24, в являются вершина 5 с исходящей дугой 5-6 (путь 4) и вершина 203 с исходящей дугой 203-204. Наличие таких вершин свидетельствует о существовании в графе еще одной группы взаимосвязанных путей, расчеты для которой также выполняются в три этапа изложенным выше порядком.

Отсутствие подобных вершин в полученном остовном дереве означает завершение его построения и окончание всех расчетов. 


\section{ГЛАВА 5}

\section{РАСЧЕТ ГОРОЧНЫХ ГОРЛОВИН СОРТИРОВОЧНЫХ ПАРКОВ}

\section{1. Расчет углов поворота дополнительных кривых}

Для определения неизвестного угла $\beta$ на расчетном пути разработан итерационный метод [30], который не требует составления и преобразования сложных тригонометрических уравнений и поэтому при автоматизации расчетов имеет существенное преимущество перед традиционными методами. Сущность итерационного метода заключается в следующем. Первоначально принимается некоторое начальное значение угла $\beta=\beta_{0}$ и при этом значении вычисляется ордината расчетного пути как сумма проекций элементов этого пути на ось $O Y$; очевидно указанная ордината является функцией угла $\beta$ :

$$
y\left(\beta_{0}\right)=y_{1}+\sum_{i=1}^{n} l_{i} \sin \theta_{i},
$$

где $y_{1}$ - ордината начального стрелочного перевода;

$l_{i}-$ длина элемента;

$\theta_{i}-$ угол наклона элемента к оси абсцисс;

$n$ - число элементов расчетного пути.

Следует заметить, что расчетный путь любой конфигурации можно представить в виде ломаной линии, если кривые заменить их тангенсами

$$
l_{i}=T_{i-1}+f_{i-1,}, i+T_{i},
$$

где $T_{i-1}, T_{i}-$ тангенсы кривых в $(i-1)$-й и $i$-й вершинах;

$f_{i-1, i}$ - длина заданной прямой вставки между $(i-1)$-й и $i$-й вершинами.

Угол наклона $i$-го элемента $\theta_{i}$ можно найти как алгебраическую сумму углов поворота в точках расчетного пути

$$
\theta_{i}=\varphi+\sum_{j=0}^{i-1} \alpha_{j},
$$

где $\quad \varphi-$ угол наклона базисного элемента;

$\alpha_{j}-$ угол поворота расчетного пути в $j$-й точке. 
Таким образом, при некотором заданном значении $\beta_{0}$ неизвестного угла $\beta$ с помощью приведенных выражений можно вычислить величину $y\left(\beta_{0}\right)$. Вычисленное значение $y\left(\beta_{0}\right)$ сравнивается с ординатой расчетного пути $Y$ и в зависимости от результатов сравнения изменяется на заданную величину $\Delta \beta$ (шаг итерации) предыдущее значение угла $\beta$ :

$$
\beta_{k+1}=\beta_{k}+\Delta \beta \cdot \operatorname{sign}\left(Y-y\left(\beta_{k}\right)\right), k=0,1,2, \ldots
$$

Указанная процедура повторяется до тех пор, пока не будет найден интервал $\left[\beta_{\kappa}, \beta_{k+1}\right]$, в котором величина $Y-y(\beta)$ меняет знак. После этого поиск угла осуществляется внутри указанного интервала методом половинного деления; расчеты завершаются при выполнении условия

$$
\left|Y-y\left(\beta_{k}\right)\right|-\varepsilon \leq 0,
$$

где $\varepsilon$-требуемая точность определения ординаты расчетного пути.

Многочисленные расчеты подтвердили эффективность данного алгоритма.

Следует заметить, что в горловине может быть несколько неизвестных углов, для каждого из которых должен быть задан отдельный расчетный путь; расчет указанных углов осуществляется поочередно.

\section{2. Расчет элементов плана горочной горловины}

После определения неизвестных углов $\beta$ рассчитываются параметры всех кривых (тангенс $T$, длина кривой $K$ ), а также координаты $x, y$ ЦП и ВУП всей горловины. При этом возможны два различных метода расчета: метод обхода дерева схемы $G_{\text {г }}=(V, E)$ и метод, основанный на предварительном описании конструкции пучков путей.

Первый метод предполагает обход элементов дерева $G_{\text {г }}$, начиная с его корня (вершина $r$ ). При этом для расчета координат $x, y$ используются рекуррентные выражения вида (5.1)-(5.3). Данный метод требует задания параметров всех элементов пучков путей, однако, он является универсальным и его целесообразно использовать при наличии в горловине нестандартных пучков. 
Второй метод основан на предварительном представлении конструкции пучков путей с помощью методов аналитической геометрии. Идея метода заключается в том, что конструкция стрелочной зоны пучка путей инвариантна относительно его положения в горловине. В этой связи описание конструкции стандартных пучков путей может постоянно храниться в базе данных программы расчета. Тогда в процессе проектирования конкретной горловины необходимо лишь установить ее пучки в требуемое положение и затем рассчитать необходимые параметры всех элементов пучков. При этом необходимое положение пучков определяется при расчете неизвестных углов $\beta$ на расчетных путях. Данный метод целесообразно применять при проектировании горочных горловин со стандартными пучками. Сущность метода заключается в следующем [31]. Конструкция любого пучка путей может быть описана совокупностью координат ЦП и ВУП $x_{i}, y_{i}$ его стрелочной зоны, а также уравнений прямых, являющихся осями путей оконечных стрелочных переводов пучка (см. рис. 5.1).

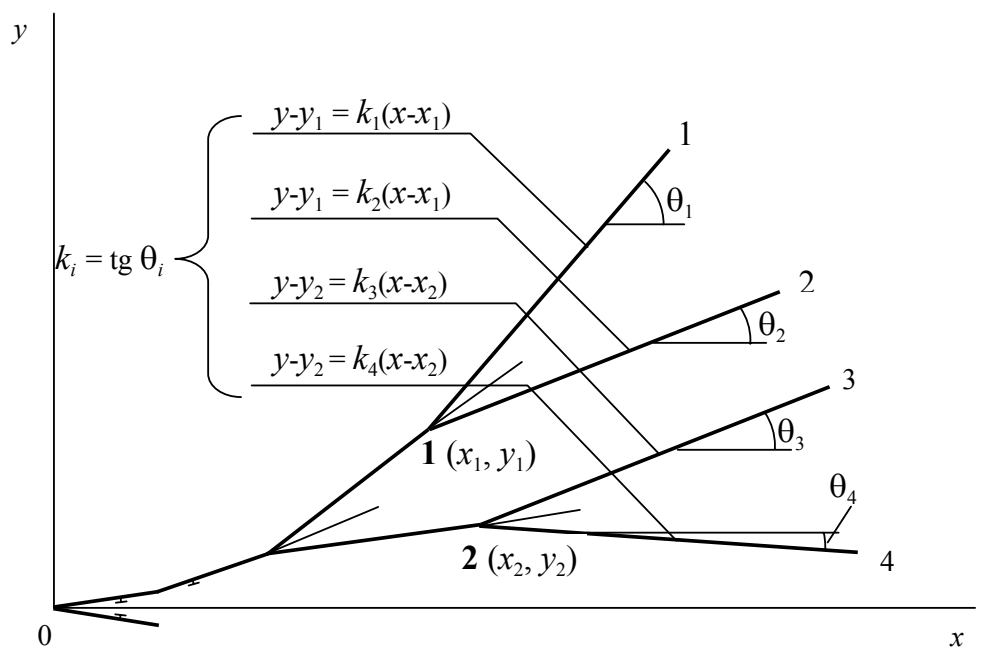

Рисунок 5.1 - Уравнения путей 1-4 в системе координат пучка Oxy горочной горловины 
При этом значения координат $\left(x_{i}, y_{i}\right)$ определяются в системе координат пучка $O x y$; уравнения указанных прямых представляются в форме

$$
y-y_{i}=k_{j}\left(x-x_{i}\right), \quad i=1, \ldots, n_{\mathrm{c}} ; j=1, \ldots, M_{\text {п }}
$$

где $x_{i}, y_{i}-$ координаты ЦП, через который проходит прямая;

$k_{j}$ - угловой коэффициент прямой $\left(k_{j}=\operatorname{tg} \theta_{j}\right)$;

$\theta_{j}$ - угол наклона прямой к оси пучка $x$;

$n_{\mathrm{c}}$ - число оконечных стрелочных переводов в пучке;

$M_{\text {п }}$ - число путей в пучке.

Найти координаты ВУП на всех путях пучка, координаты ЦП, а также параметров соединительных кривых можно, используя методы аналитической геометрии. Расчет осуществляется для всех путей отдельного пучка, головная стрелка которого находится в точке $\left(X_{0}, Y_{0}\right)$, а угол между осью пучка и осью сортировочного парка равен $\varphi$. Для расчётов необходимо выполнить следующие процедуры:

1. Поместить начало координат пучка $x 0 y$ в заданную точку горловины $\left(X_{0}, Y_{0}\right)$ в системе координат сортировочного парка $O X Y$.

2. Осуществить поворот координатных осей $O x y$ на требуемый угол $\varphi$ так, чтобы ось $x$ совпала с осью пучка в системе координат $O X Y$.

3. Найти координаты ЦП и ВУ $\left(X_{i}, Y_{i}\right)$ стрелочной зоны пучка в системе координат $O X Y$.

4. Выполнить преобразование уравнений осей путей (5.4) из системы координат пучка $O x y$ в систему координат сортировочного парка $O X Y$.

5. Найти точки пересечения осей путей пучка и осей соответствующих сортировочных путей и найти координаты вершин углов поворота $\left(X_{\text {вуj}}, Y_{\text {вуj }}\right)$.

6. Найти расстояния $l_{j}$ между соответствующими ЦП и ВУ на сортировочных путях.

7. Определить параметры соединительных кривых (угол поворота $\beta_{j}$, тангенс $T_{j}$, длину $K_{j}$ ) на сортировочных путях.

8. Определить величины прямых вставок $F_{j}$ между концами крестовин и началами соответствующих соединительных кривых. 
Все использованные величины показаны на рис. 5.2, где графически выполнены указанные преобразования для $j$ - го пути пучка.

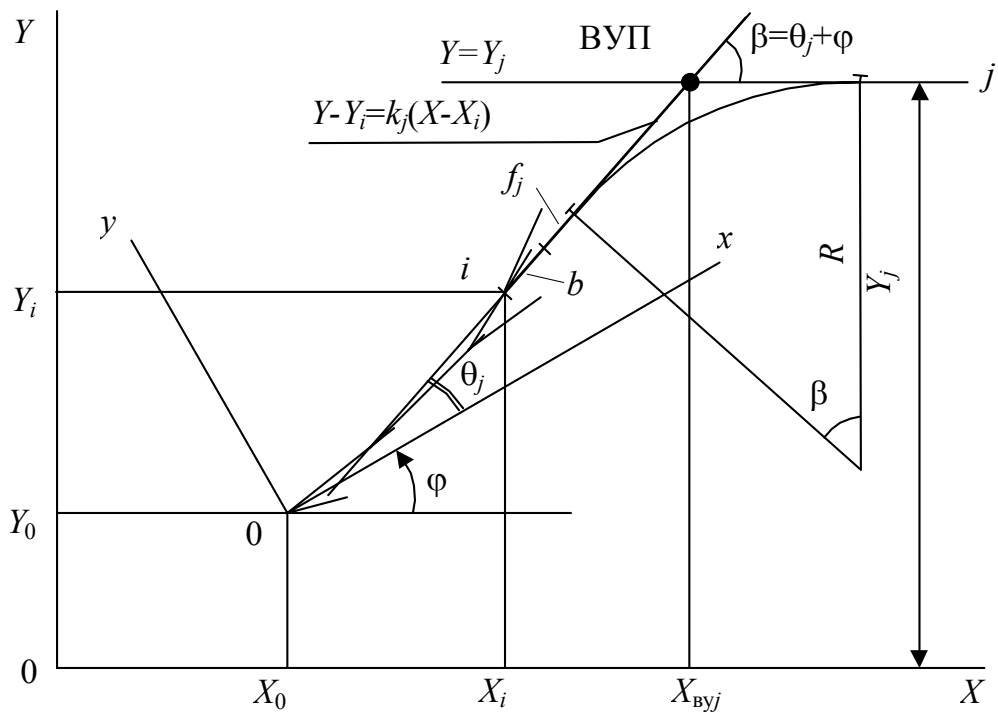

Рисунок 5.2 - Схема определения координат вершины угла поворота на j-том сортировочном пути

Перечисленные выше процедуры выполняются аналитически с использованием следующих выражений. Расчёт координат ЦП и ВУ $\left(X_{i}, Y_{i}\right)$ в системе координат сортировочного парка $X 0 Y$ по известным координатам эти точек $\left(x_{i}, y_{i}\right)$ в системе координат пучка $O x y$ осуществляется с помощью выражений:

$$
\begin{aligned}
& X_{i}=X_{0}+x_{i} \cos \varphi-y_{i} \sin \varphi, \\
& Y_{i}=Y_{0}+x_{i} \sin \varphi+y_{i} \cos \varphi .
\end{aligned}
$$

Преобразование уравнений осей путей пучка из системы $O x y$ в систему $О Х У$ осуществляется следующим образом. Вначале определяются угловые коэффициенты указанных прямых $k_{j}$ в новой системе $O X Y$ :

$$
k_{j}=\operatorname{tg}\left(\theta_{j}+\varphi\right) .
$$


Затем записываются собственно уравнения прямых в форме (5.4) но с учетом преобразованных координат $X_{i}$ и $Y_{i}$ :

$$
Y-Y_{i}=k_{j}\left(X-X_{i}\right)
$$

Тогда ВУ соединительной кривой можно найти как точку пересечения прямой (5.5) и прямой $Y=Y_{j}$ (см. рис. 5.2). Координаты указанной точки $\left(X_{\text {вуj }}, Y_{\text {вуj }}\right)$ можно найти, решая совместно уравнения указанных прямых:

$$
\left.\begin{array}{l}
Y-Y_{i}=k_{j}\left(X-X_{i}\right) \\
Y=Y_{j}
\end{array}\right\},
$$

где $Y_{j}$ - ордината соответствующего сортировочного пути (в системе $O X Y$ ). Отсюда координаты ВУ:

$$
\begin{aligned}
& X_{\text {ву } j}=\frac{Y_{j}-Y_{i}}{k_{j}}+X_{i}, \\
& Y_{\text {ву } j}=Y_{j} .
\end{aligned}
$$

Расстояния $l_{j}$ между ВУ и ЦП можно найти по координатам соответствующих точек как

$$
l_{j}=\sqrt{\left(X_{\mathrm{Byj}}-X_{i}\right)^{2}+\left(Y_{\mathrm{By} j}-Y_{i}\right)^{2}} .
$$

Параметры соединительных кривых определяются по известным формулам:

$$
\begin{gathered}
\beta_{j}=\theta_{j}+\varphi, \\
T_{j}=R_{j} \operatorname{tg} \frac{\beta_{j}}{2}, \\
K_{j}=\frac{\pi R_{j} \beta_{j}}{180^{\circ}} .
\end{gathered}
$$

Здесь $R_{j}$ - принятое значение радиуса соединительной кривой на данном пути. И, наконец, определяется величина прямой вставки $f_{j}$

$$
f_{j}=l_{j}-T_{j}-b
$$

где $\quad b$-элемент стрелки.

Приведенные формулы положены в основу алгоритма, позволяющего рассчитать координаты всех ЦП и ВУ для заданного пучка путей, параметры 
всех соединительных кривых, а также величины прямых вставок между крестовинами стрелок и этими кривыми.

\section{3. Проектирование конечных соединений сортировочных путей}

При расчете параметров соединительных кривых (см. п. 5.2) на сортировочных путях, не являющихся расчетными, могут возникнуть определенные трудности [31]. В частности, в некоторых случаях величина вставки между концом крестовины и закрестовинной кривой оказывается отрицательной даже при минимальном радиусе соединительной кривой (см. рис. $5.3, a$ ). В других случаях расчетная ВУП соединительной кривой (точка А) вообще находится до соответствующего ЦП (см рис. 5.3, б). В обеих приведенных случаях расчетная ВУП (точка А) является фиктивной; в действительности сопряжение стрелочного перевода и сортировочного пути осуществляется

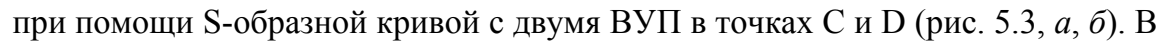
этих случаях автоматически рассчитываются параметры соединительной $\mathrm{S}$-образной кривой при заданном радиусе $R$ и нулевой вставке между обратными кривыми. Подобное сопряжение при помощи S-образной кривой может потребоваться и в тех случаях, когда при использовании одной круговой кривой не выдерживается минимальная ширина междупутья для смежных путей (рис. 5.3, в). Здесь так же, как и в приведенных выше случаях, найденная ВУП (точка А) заменяется двумя другими (точки С и D). И, наконец, по тем же соображениям в необходимых случаях одну соединительную кривую заменяют двумя кривыми одного направления (рис. 5.3, г).

Анализ четырех приведенных случаев показывает, что задача поиска оптимальных параметров соединительных кривых достаточно сложна. Как видно из рис. 5.4, соединительную кривую в общем случае можно описать нелинейным уравнением, включающим 4 независимых переменных:

$\Delta Y=\left(b+f_{1}\right) \sin \theta+R_{1} \cos \theta-R_{1} \cos (\theta+\beta)+f_{2} \sin (\theta+\beta)+R_{2}-R_{2} \cos (\theta+\beta)$ 
a)

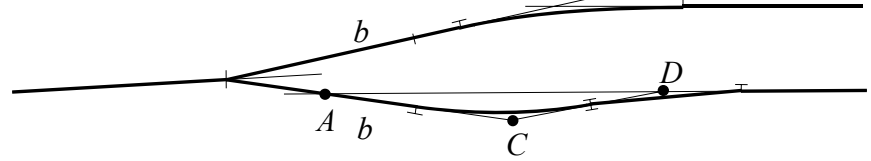

б)

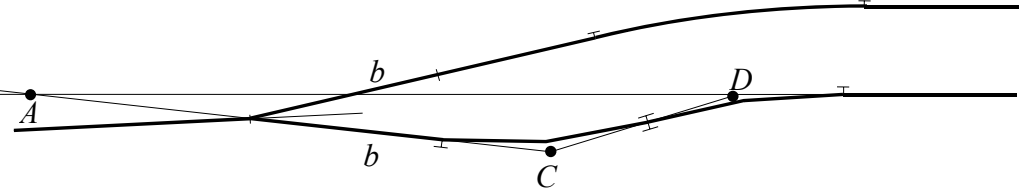

в)

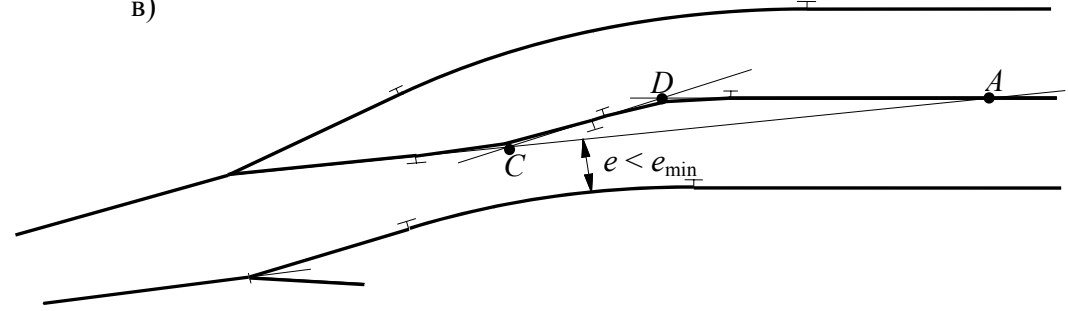

г) $\quad e<e_{\min }$

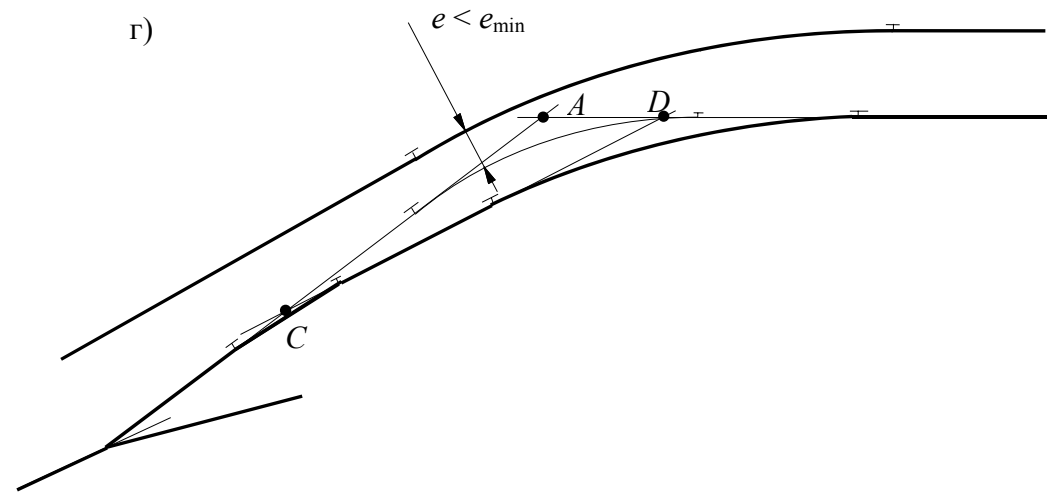

Рис. 5.3 Соединительные кривые на сортировочных путях

В качестве независимых переменных удобно принять $f_{1}, \beta, R_{1}, R_{2}$; величины $\Delta Y$ и $\theta$ известны, а величина вставки $f_{2}$ при этом находится из данного выражения. 
Следует заметить, что указанную задачу нельзя решать для отдельно взятого пути, поскольку его положение влияет на ширину междупутий со смежными путями. Следовательно, ее необходимо решать, как минимум, для отдельного пучка путей. В качестве критерия оптимальности можно принять максимальное расстояние $L_{i}$ от головной стрелки пучка до предельного столбика или до точки возможной установки замедлителей на путях пучка, которое необходимо минимизировать $\left(\max \left\{L_{i}\right\} \rightarrow \min , i=1, \ldots, M_{\text {п }}\right)$.

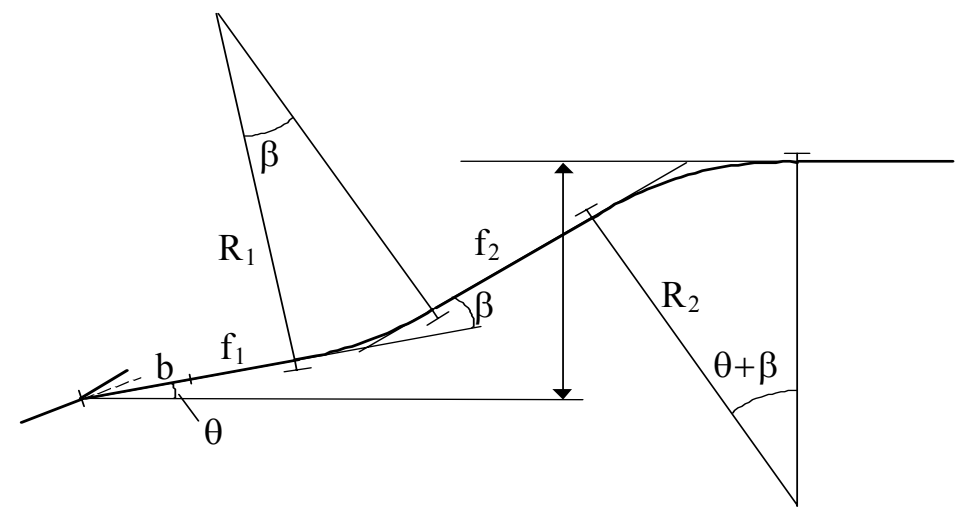

Рисунок 5.4. - Параметры $S$-образной соединительной кривой сортировочного пути

Таким образом, сформулированная задача относится к негладким задачам нелинейного программирования, точное решение которой получить весьма затруднительно. В то же время проектировщик может достаточно быстро найти приближенное решение данной задачи, дающее удовлетворительные результаты, при наличии необходимой информации. В этой связи в разработанной методике синтеза горочных горловин предусмотрен интерактивный режим выбора трассы сортировочных путей. В этом режиме имеется возможность изменения четырех указанных переменных $f, \beta, R_{1}, R_{2}$ с одновременным отображением на дисплее его результатов. После изменения значений указанных параметров для некоторого пути на дисплее корректируется положение точек, расстояние от которых до осей смежных путей достигает 
величины $g_{\text {min }} / 2$, достаточной для установки замедлителя (предельного столбика). Методика определения положения указанных точек изложена в п. 5.4.

\section{4. Определение положения замедлителей}

При проектировании горловин сортировочных парков возникает задача определения положения парковых тормозных позиций (ПТП) на сортировочных пучках. В соответствии с [32], замедлители РНЗ могут размещаться в кривых радиуса 180 м и более в местах, где ширина междупутья не менее заданной величины $g_{m i n}$. В этой связи для проектирования размещения ПТП необходимо прежде всего определить по каждому пути расстояние от ЦП до точки, в которой ширина междупутья достигает $g_{m i n}$. Данную задачу необходимо решать при выборе трассы сортировочных путей в интерактивном режиме (см. п. 5.3), и поэтому она нуждается в автоматизации. Расчет указанных расстояний может быть выполнен методами аналитической геометрии с помощью формул, аналогичных используемым для определения положения предельных столбиков и сигналов [1]. Следует учесть, однако, что сложность конструкции горочных горловин, в которых начальная часть сортировочных путей представляет собой комбинацию из прямолинейных отрезков и кривых разного направления, радиуса и длины, весьма затрудняет формализацию указанных расчетов и потребует значительной ручной подготовительной работы. В этой связи для автоматизации решения поставленной задачи целесообразно использовать современные методы вычислительной геометрии, позволяющие решить данную задачу для сортировочных путей с произвольной трассой. Точка $P$, в которой расстояния до смежных сортировочных путей

равны $\Delta=g_{\text {min }} / 2$, является точкой пересечения двух эквидистант, построенных для каждого из рассматриваемых путей в их общем междупутье. Эквидистанта определяется как геометрическое место точек, которые являются концами нормалей длиной $\Delta$, восстановленных в каждой точке исходной кривой [33].

Каждый сортировочный путь может быть представлен кусочно-гладкой 
кривой, состоящей из отрезков и дуг ориентированных окружностей (см. рис. 5.5). Как показывает анализ выполненных проектов горочных горловин [34], на пути может быть одна или две круговых кривых и, соответственно, два или три отрезка.

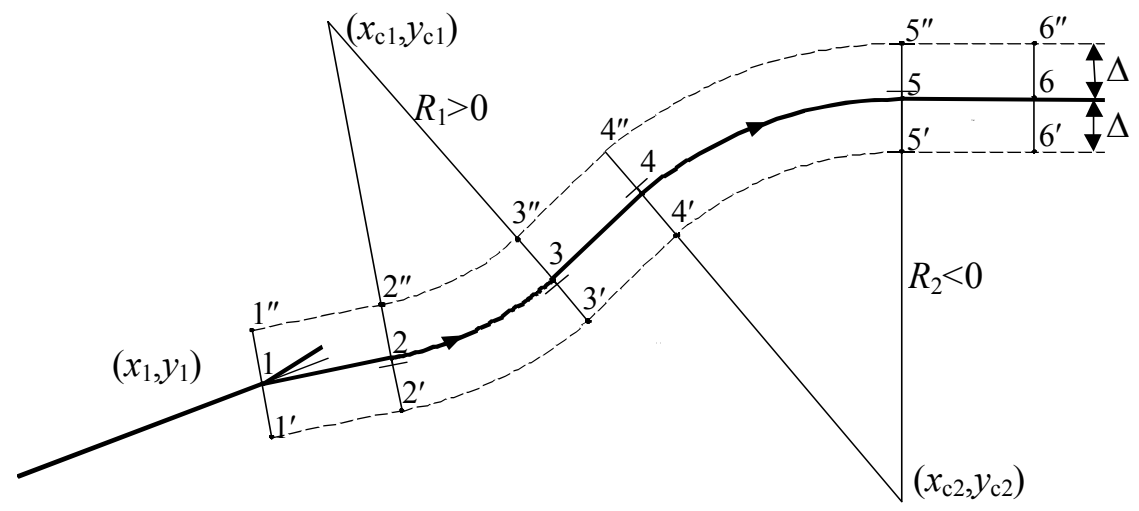

Рисунок 5.5 - Эквидистанты сортировочного пути

Для однозначного описания кусочно-гладкой кривой достаточно задать список координат концов отрезков $\left(x_{1}, y_{1}\right), \ldots,\left(x_{6}, y_{6}\right)$, начиная от стрелочного перевода, а также параметры ориентированных окружностей $\left(x_{\mathrm{c}}, y_{\mathrm{c}}, R\right)$. При этом ориентацию окружности можно передать знаком ее радиуса: радиусу ориентированной окружности присваивается знак плюс (минус), если движение вдоль нее в направлении от стрелочного перевода совершается против (по) часовой стрелке [33]. Данная модель кусочно-гладкой кривой удобна для построения эквидистант сортировочных путей. Для этого достаточно выполнить параллельный перенос отрезков 1-2, 3-4, 5-6 на расстояние $\pm \Delta$ (вычислить координаты их концов 1'-6' и 1"-6" после переноса) и изменить на величину $\Delta$ радиусы кривых $\left(R^{\prime}=R+\Delta, R^{\prime \prime}=R-\Delta\right.$ с учетом их знака (см. рис. 5.5)). Для нахождения искомой точки $P$ между двумя смежными путями необходимо для верхнего пути построить эквидистанту, отстоящую от него на $+\Delta$, для нижнего пути - на $-\Delta$, и найти точку их пересечения (см. рис. 5.6). 


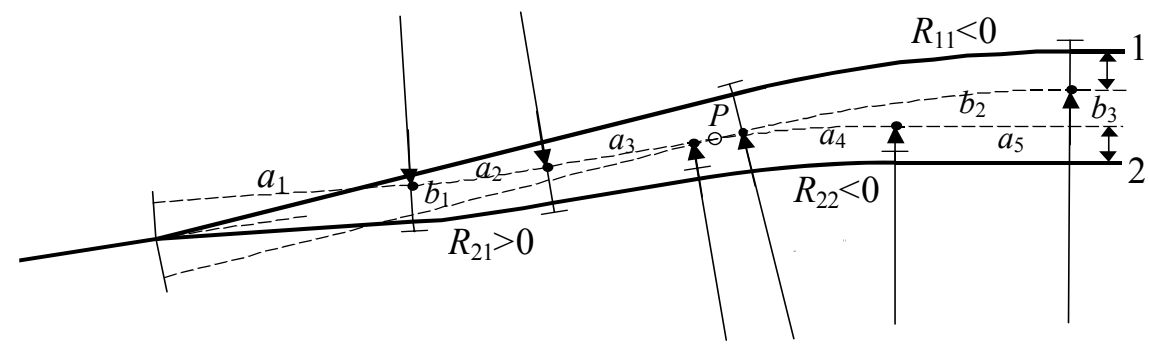

Рисунок 5.6 - Определение точки $P$, отстоящей от смежных путей на расстояние $\Delta$

Для нахождения точки пересечения эквидистант необходимо поочередно рассматривать возможность пересечения каждого элемента одной из них с каждым элементом другой. Таким образом, возникают задачи определения точек пересечения двух отрезков, отрезка и дуги, а также двух дуг. Для определения точки пересечения двух отрезков использован эффективный метод вычислительной геометрии, основанный на представлении отрезков уравнениями в параметрической форме [28]. Для двух других задач разработаны оригинальные алгоритмы (см. п. 5.5), также основанные на методах вычислительной геометрии.

\section{5. Поиск точек пересечения эквидистант смежных сортировоч- ных путей}

Алгоритм основан на поочередном поиске возможной точки пересечения каждого элемента эквидистанты одного пути с каждым элементом эквидистанты смежного пути. При этом можно сократить объем вычислений, если не рассматривать те элементы эквидистант, которые заведомо не имеют общих точек. Для описания, предлагаемой схемы перебора обозначим элементы эквидистант нижнего пути как $p_{i}, i=1, \ldots, m$, верхнего пути - как $q_{j}$, $j=1, \ldots, n$, а процедуру определения точки пересечения элементов $-\psi\left(p_{i}, q_{j}\right)$. Обозначим также абсциссу левого конца элемента как $x_{l}\left(p_{i}\right)$, правого $-x_{r}\left(p_{i}\right)$. Тогда алгоритм перебора можно представить в виде следующих четырех шагов: 
1. Начальная установка $i=1$ и $j=1$.

2. Пока $i \leq n$ и $x_{r}\left(p_{i}\right) \geq x_{l}\left(q_{j}\right)$, то $\psi\left(p_{i}, q_{j}\right)$ и $i=i+1$.

3. Корректирование индексов элементов $i=i-1, j=j+1$.

4. Если $i<n$ и $j \leq m$, то переход к 2 , иначе конец алгоритма.

Как показали исследования, использование данного алгоритма позволяет в 2-3 раза сократить объем вычислений, что весьма важно в интерактивном режиме проектирования соединений сортировочных путей. Для реализации алгоритма были разработаны методы определения точек пересечения элементов эквидистант (двух отрезков, дуги и отрезка, двух дуг), основанные на методах вычислительной геометрии [28].

Для нахождения точки пересечения двух отрезков уравнение одного из них, проходящего через точки $a\left(x_{a}, y_{a}\right)$ и в $\left(x_{b}, y_{b}\right)$, должно быть записано в параметрической форме

$$
\left.\begin{array}{l}
x=x_{a}+t\left(x_{b}-x_{a}\right) \\
y=y_{a}+t\left(y_{b}-y_{a}\right)
\end{array}\right\},
$$

где параметр $t$ может принимать любое значение в диапазоне $0 \leq t \leq 1$.

Для нахождения точки пересечения данного отрезка с отрезком, проходящим через точки $c\left(x_{c}, y_{c}\right)$ и $d\left(x_{d}, y_{d}\right)$ (см. рис. 5.7), необходимо найти значение параметра $t$ вдоль отрезка $\mathbf{a b}$, соответствующее точке пересечения [28]:

$$
t=\frac{\left(y_{d}-y_{c}\right)\left(x_{a}-x_{c}\right)+\left(x_{c}-x_{d}\right)\left(y_{a}-y_{c}\right)}{\left(y_{d}-y_{c}\right)\left(x_{b}-x_{a}\right)+\left(x_{c}-x_{d}\right)\left(y_{b}-y_{a}\right)} .
$$

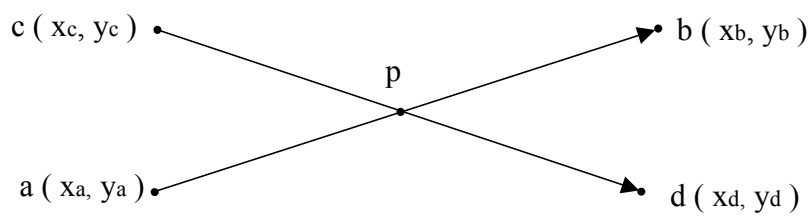

Рисунок 5.7 - Нахождение точки пересечения двух отрезков $\mathbf{a b}$ и $\mathbf{c d}$, заданных координатами их границ

Если в данном выражении знаменатель равен нулю, то отрезки совпадают или параллельны; иначе, если параметр $t$ находится в диапазоне $0 \leq t \leq 1$, то 
отрезок $\mathbf{a b}$ пересекает прямую, проходящую через точки $c$ и $d$. В этом случае координаты точки пересечения $p\left(x_{p}, \mathrm{y}_{p}\right)$ можно найти, зная $t$, с помощью уравнения (5.7). При этом точка пересечения инцидентна отрезку сd, если выполняется условие

$$
\left(x_{p}-x_{c}\right)\left(x_{d}-x_{p}\right)>0 .
$$

Если же отрезок $\mathbf{c d}$ вертикален, то условие принадлежности этому отрезку точки $p$ может быть записано в виде

$$
\left(y_{p}-y_{c}\right)\left(y_{d}-y_{p}\right)>0 .
$$

Для нахождения точек пересечения дуги и отрезка необходимо найти точки пересечения $p, q$ отрезка $\mathbf{a b}$ с ориентированной дугой $r \breve{s}$ радиуса $R$ с центром в точке $c\left(x_{c}, y_{c}\right)$ (см. рис. 5.8). Построим отрезок единичной длины cd, равный отрезку ab и перпендикулярный ему $\mathbf{c}$ началом в центре дуги $c\left(x_{c}, y_{c}\right)$. С этой целью необходимо повернуть отрезок $\mathbf{a b}$ на $90^{\circ}$ по часовой стрелке относительно точки $a$ и переместить его из точки $a$ в центр дуги (точка $c$ ). При этом координаты другого конца отрезка сd (точки $d$ ) можно найти как [28]:

$$
\left.\begin{array}{l}
x_{d}=y_{b}-y_{a}+x_{c} \\
y_{d}=x_{a}-x_{b}+y_{c}
\end{array}\right\} .
$$

Тогда с помощью (5.8) можно найти значение параметра $t$ вдоль отрезка $\mathbf{a b}$, соответствующее точке пересечения прямых $\mathbf{a b}$ и $\mathbf{c d}$ (рис. 5.8, точка e):

$$
t=\frac{\left(x_{a}-x_{b}\right)\left(x_{a}-x_{c}\right)+\left(y_{a}-y_{b}\right)\left(y_{a}-y_{c}\right)}{\left(x_{a}-x_{b}\right)^{2}+\left(y_{a}-y_{b}\right)^{2}} .
$$

Координаты точки $e\left(x_{e}, y_{e}\right)$ можно найти с помощью (5.7); тогда длину отрезка ре, очевидно, можно найти как:

$$
L_{p e}=\sqrt{R^{2}-\left(x_{c}-x_{e}\right)^{2}-\left(y_{c}-y_{e}\right)^{2}} .
$$

Если в (5.12) выражение под знаком корня отрицательно, то прямая $\mathbf{a b}$ и окружность, являющаяся носителем дуги $r \breve{s}$, не пересекается. В противном случае точки пересечения $p$ и $q$ расположены симметрично относительно точки $e$. Для нахождения координат точки $q$ переместим единичный отрезок $\mathbf{a b}$ в точку $e$; для нахождения координат точки $p$ переместим в точку $e$ отре- 
зок $\mathbf{a b}$, повернутый вокруг точки $a$ на $180^{\circ}$. Тогда положение точек $p$ и $q$ на этих отрезках можно определить параметром $\pm \tau$, величина, которого равна отношению длин отрезков pe (qe) и $\mathbf{a b :}$

$$
\tau= \pm L_{p e} / \sqrt{\left(x_{a}-x_{b}\right)^{2}+\left(y_{a}-y_{b}\right)^{2}}
$$

а их координаты можно найти с помощью параметрических уравнений вида (5.7):

$$
\left.\begin{array}{l}
x_{q, p}=x_{e} \pm \tau\left(x_{b}-x_{a}\right) \\
y_{q, p}=y_{e} \pm \tau\left(y_{b}-y_{a}\right)
\end{array}\right\} .
$$

Очевидно, что отрезок ab и дуга $r \breve{s}$ пересекаются, если точки $p$ и/или $q$ инцидентны как отрезку ab, так и дуге $r \breve{s}$. Инцидентность точек отрезку проверяется с помощью выражений вида (5.9), (5.10). Для установления инцидентности точки $p$ дуге ориентированной окружности проводится ориентированная прямая $r s$ от начальной точки дуги $r$ к ее конечной точке $s$.

Если точка $p\left(x_{p}, y_{p}\right)$ принадлежит дуге, то при $R>0(R<0)$ она лежит справа (слева) от ориентированной прямой rs. Поэтому точка $p$ принадлежит дуге $r \breve{s}$, если выполняется условие [33]:

$$
R\left(\left(y_{p}-y_{r}\right)\left(x_{r}-x_{s}\right)-\left(x_{p}-x_{r}\right)\left(y_{r}-y_{s}\right)\right) \geq 0 .
$$

Проверка инцидентности точки $q$ дуге $r \breve{s}$ выполняется аналогично.

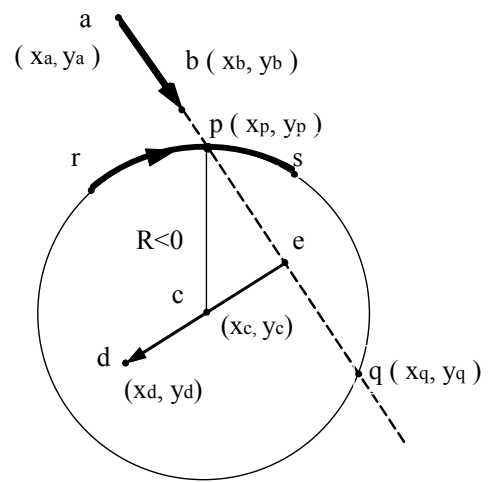

Рисунок 5.8 - Нахождение точки пересечения отрезка ab и дуги $r \breve{s}$

Для нахождение точек пересечения двух дуг необходимо найти точки пересечения $p, q$ двух ориентированных дуг $r \breve{s}$ и $u \breve{v}$ радиуса $R_{1}$ и $R_{2}$ с цен- 
трами в точках $a\left(x_{a}, y_{a}\right)$ и $b\left(x_{b}, y_{b}\right)$ соответственно (см. рис. 5.9). Построим единичные отрезки ed и ec, равные расстоянию между центрами дуг (отрезок $\mathbf{a b )}$ и перпендикулярные отрезку ab. С этой целью необходимо отрезок $\mathbf{a b}$ повернуть на $90^{\circ}$ по часовой стрелке и переместить его начало из точки $a$ в точку $e$ (отрезок ес); тогда координаты точки $c$ можно найти как (5.11):

$$
\left.\begin{array}{l}
x_{c}=y_{b}-y_{a}+x_{e} \\
y_{c}=x_{a}-x_{b}+y_{e}
\end{array}\right\} .
$$

Отрезок ed получают аналогично поворотом отрезка $\mathbf{a b}$ на $90^{\circ}$ против часовой стрелки; координаты точки $d$ :

$$
\left.\begin{array}{l}
x_{d}=-\left(y_{b}-y_{a}\right)+x_{e}=y_{a}-y_{b}+x_{e} \\
y_{d}=-\left(x_{a}-x_{b}\right)+y_{e}=x_{b}-x_{a}+y_{e}
\end{array}\right\} .
$$

Координаты точки $e$, лежащей на отрезке $\mathbf{a b}$, можно найти, используя параметрическое уравнение этого отрезка вида (5.7), используя в качестве параметра $t$ отношение длин отрезков $\mathbf{a e}$ и $\mathbf{a b}$ :

$$
t=L / D \text {, }
$$

где $\quad L-$ длина отрезка аe $L=\left(R_{1}^{2}-R_{2}^{2}+D^{2}\right) / 2 D$;

$D$ - длина отрезка $\mathbf{a b}$ (расстояние между центрами дуг)

$$
D=\sqrt{\left(x_{a}-x_{b}\right)^{2}+\left(y_{a}-y_{b}\right)^{2}} .
$$

Тогда координаты точек $p$ и $q$, расположенных соответственно на отрезках ес и ed, можно найти, используя параметрические уравнения этих отрезков вида (5.7).

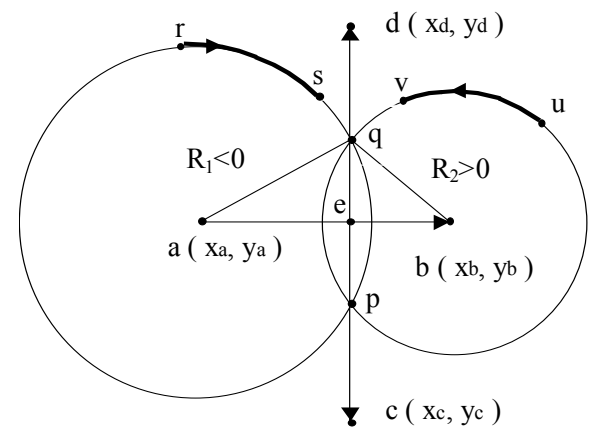

Рисунок 5.9 - Нахождение точки пересечения двух дуг $r \breve{s}$ и $u \breve{v}$ 
При этом в качестве параметра $\pm \tau$ необходимо использовать отношение длины $\lambda$ отрезков ер (eq) к длине отрезка $\mathbf{a b}(D)$ :

$$
\tau= \pm \lambda / D .
$$

Значение $\lambda$, очевидно, равно

$$
\lambda=\sqrt{R_{1}^{2}-L^{2}} .
$$

При этом, если $R_{1}^{2}-L^{2}<0$, то окружности-носители дуг не пересекаются.

После получения координат точек пересечения $p$ и $q$ окружностей, которым принадлежат дуги $r \breve{s}$ и $u \breve{v}$, необходимо проверить инцидентность каждой из этих точек обеим указанным дугам. Проверку инцидентности можно выполнить с помощью условий вида (5.13). Если точка $p(q)$ инцидентна как дуге $r \breve{s}$, так и дуге $u \breve{v}$, то дуги пересекаются в данной точке; в противном случае пересечение дуг отсутствует. 
ГЛАВА 6

ПРОГРАММНАЯ РЕАЛИЗАЦИЯ СИСТЕМЫ АВТОМАТИЗИРОВАННОГО ПРОЕКТИРОВАНИЯ ПЛАНА ПУТЕВОГО РАЗВИТИЯ СТАНЦИЙ

\section{1. Общая характеристика программного обеспечения}

На основании разработанной модели плана путевого развития и алгоритмов разработан пакет прикладных программ для автоматизированного проектирования железнодорожных станций. Указанный пакет состоит из двух программных модулей:

- программа графического ввода схем путевого развития, которая предназначена для ввода топологической структуры плана путевого развития в виде немасштабной схемы, установки конструктивных параметров и формирования входной модели;

- программа расчета координат, которая предназначена для обработки входной модели, преобразования ее во внутреннюю модель, расчета координат элементов плана путевого развития станций и формирования чертежа.

\section{2. Программа графического ввода немасштабной схемы станции}

Программа графического ввода плана путевого развития реализована на основе графического пакета AutoCAD. План путевого развития составляется из графических примитивов AutoCAD, при этом объектам плана путевого развития соответствуют следующие графические примитивы:

- участки путей - графические примитивы LINE;

- стрелочные переводы - блоки SWITCH;

- вершины углов поворота - блоки CURVE;

- номера путей - блоки NUMWAY;

- указатель ширины междупутья - блоки MIDWAY.

Передача конструктивных параметров осуществляется с помощью атрибутов блоков и расширенных данных AutoCAD.

Ввод и редактирование немасштабной схемы осуществляется с помо- 
щью специально разработанной системы команд. Вызов команд осуществляется с помощью командной строки или панели инструментов PLAN (см. рис. 6.1).

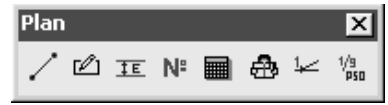

Рисунок 6.1 - Панель инструментов PLAN

Ввод плана путевого развития осуществляется с помощью команды PL-LINE ( $\nearrow$ ). Указанная команда выдает следующие запросы:

\section{Command: PL-LINE \\ Введите начальную точку: \\ Введите конечную точку:}

В ответ на данные запросы необходимо левым щелчком мыши указать на экране необходимую точку.

При вводе очередного путевого участка осуществляется контроль его конечных точек. На основании количества сходящихся в этих точках отрезков определяется степень соответствующих им вершин графа плана путевого развития. В случае, если степень вершины составляет 2 в данную точку вставляется блок CURVE, а если степень вершины составляет 3 - блок SWITCH, предназначенные для хранения конструктивных параметров соответствующих элементов плана путевого развития. Для поддержания целостности модели используется механизм объектных реакторов AutoCAD. При этом в моменты удаления, копирования и модификации объектов LINE автоматически осуществляется контроль и изменение конечных точек отрезков в их старом и новом положении. Окно графического редактора AutoCAD в процессе ввода немасштабной схемы изображено на рис. 6.2. Ввод номеров путей и вставка указателей ширины междупутий осуществляется с помощью команд PL-NUMWAY (№ ) и PL-MIDWAY (重). 


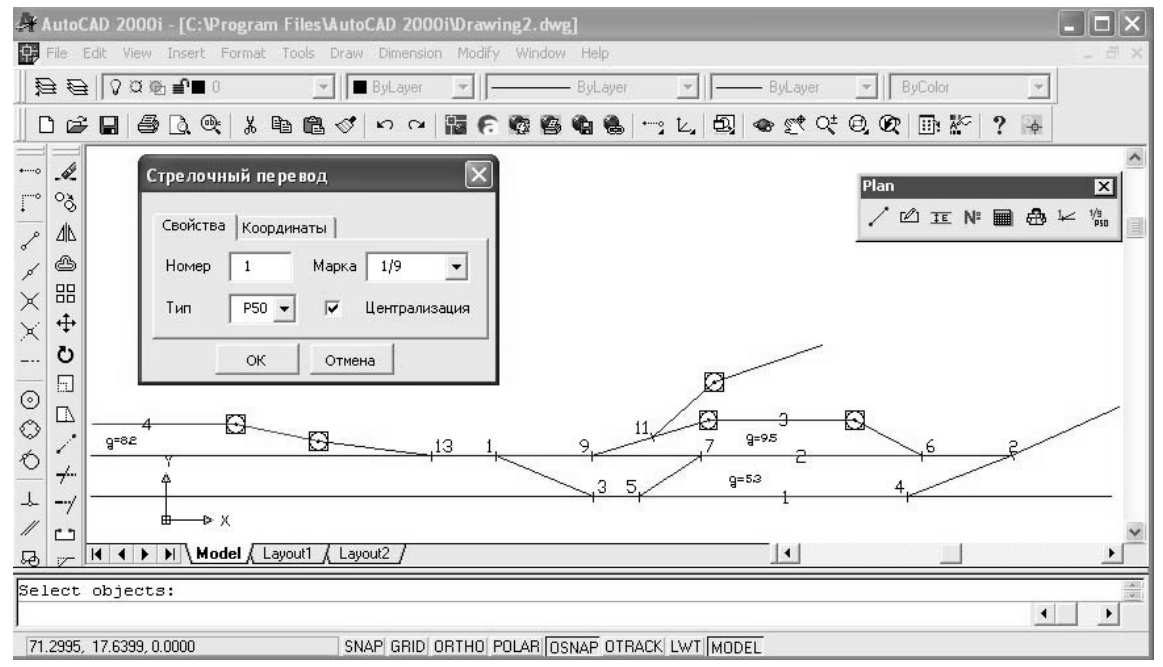

Рисунок 6.2 Проектирование плана путевого развития

При создании объектов плана путевого развития их начальные значения устанавливаются в соответствии с заданными параметрами по умолчанию. Для изменения параметров по умолчанию используется команда

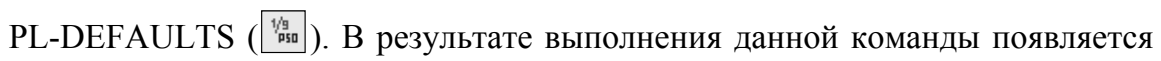
диалоговое окно "Параметры по умолчанию" (см. рис. 6.3). В данном диалоговом окне можно установить марку крестовины, тип рельса стрелочных переводов и радиус кривых, которые устанавливаются для соответствующих объектов при их создании.

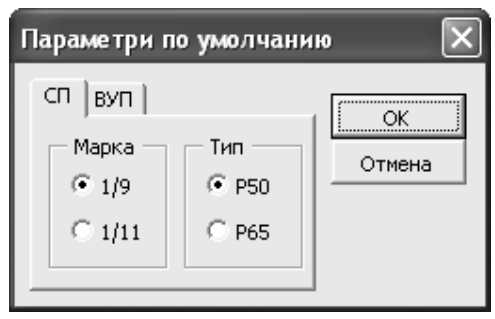

Рисунок 6.3 Диалоговое окно для изменения начальных параметров элементов плана путевого развития

Команда PL-NUMERATION ( $\longleftarrow$ ) позволяет установить режим четной и нечетной нумерации элементов плана путевого развития. 
Изменение конструктивных параметров выполняется с помощью команды PL-EDIT (ø). Указанная команда выдает следующие запросы:

\section{Command: PL-EDIT \\ Select objects:}

В ответ на указанный вопрос необходимо выбрать объект или группу однотипных объектов плана путевого развития и нажать клавишу ENTER. B результате будет открыто диалоговое окно для изменения параметров. Внешний вид этого окна зависит от того, какой элемент был выбран (см. рис. 6.4).
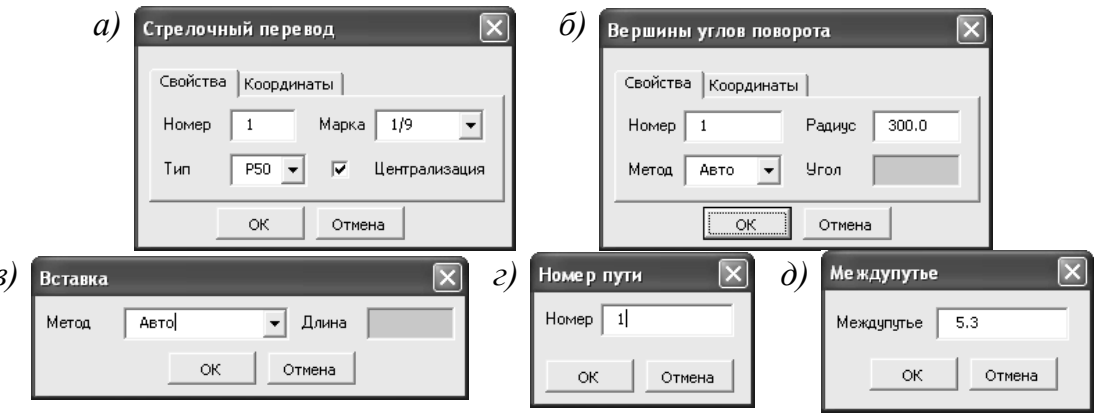

Рисунок 6.4 - Диалоговые окна для редактирования параметров элементов плана путевого развития: $a$ - стрелочных переводов; $\sigma$ - кривых; $b$ - участков пути; 2 - номеров пути; $\partial$ - указателей междупутий

На вкладке “Координаты” могут быть заданы мировые координаты точки, которые не будут изменяться при расчете.

В случае выбора для редактирования группы однотипных объектов те поля, значения которых различаются, устанавливаются в неопределенное значение.

Расчет координат элементов плана путевого развития инициируется с помощью команды PL-CALCULATE (凅). В результате выполнения данной команды формируется файл ktb0000.stn и автоматически загружается программа SaprConv.exe. 


\section{3. Программа расчета координат точек плана путевого развития станции}

Расчет координат элементов плана путевого развития выполняется программой SaprConv.exe. Внешний вид окна данной программы показан на рис. 6.5 .

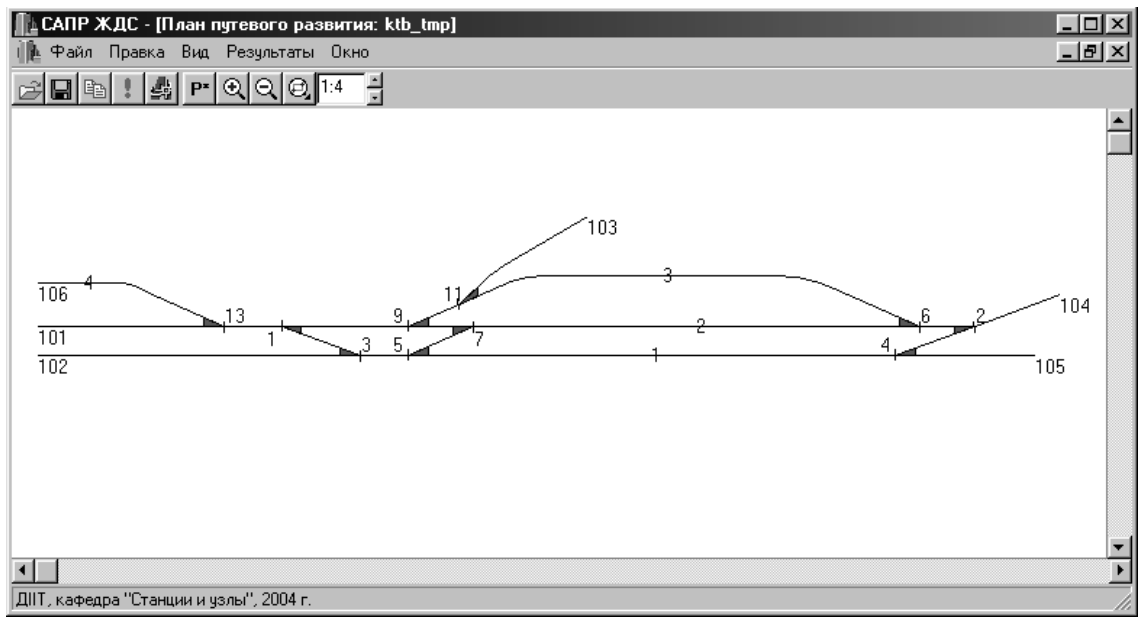

Рисунок 6.5 - Внешний вид окна программы расчета плана путевого развития станций

Управление рабой программы выполняется с помощью меню и панели инструментов.

Для того, чтобы открыть файл необходимо выполнить команду меню Файл $\rightarrow$ Открыть или нажать кнопку данных для расчета должны быть представлены в формате stn или ktb (см. Приложение А). В результате будет выполнен расчет координат схемы и построение масштабного плана. Замечания по кодированию схемы выводятся в окне «Сообщения».

Для анализа конструктивных элементов схемы необходимо выполнить команду Вид $\rightarrow$ Выделить вставки или нажать кнопку $\mathrm{P}^{\mathbf{x}}$. В результате указанных действий вставки р* будут выделены зеленым цветом, а вставки, дли- 
на которых задана проектировщиком - синим. Стрелочные переводы марки 1/9 будут залиты желтым, более пологие - красным цветом.

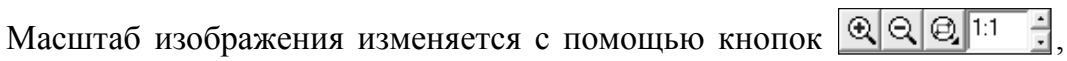
команды меню Вид $\rightarrow$ Масшаб и клавиш +, -, *, /, Home.

Программа SaprConv.exe предусматривает редактирование плана путевого развития на уровне внутренней модели. Для изменения конструктивных параметров элементов плана путевого развития (стрелочных переводов, круговых кривых, путевых вставок) необходимо выделить нужный элемент и выбрать команду контекстного меню «Свойства». При этом в зависимости от типа элемента необходимо будет открыто диалоговое окно (см. рис. 6.6) для изменения параметров. Для повторного расчета необходимо нажать кнопку !.

Корректирование модели может быть также выполнено с помощью специального редактора. Указанный редактор позволяет также выполнять логический контроль вводимых данных и в необходимых случаях выдает сообщения об ошибках.

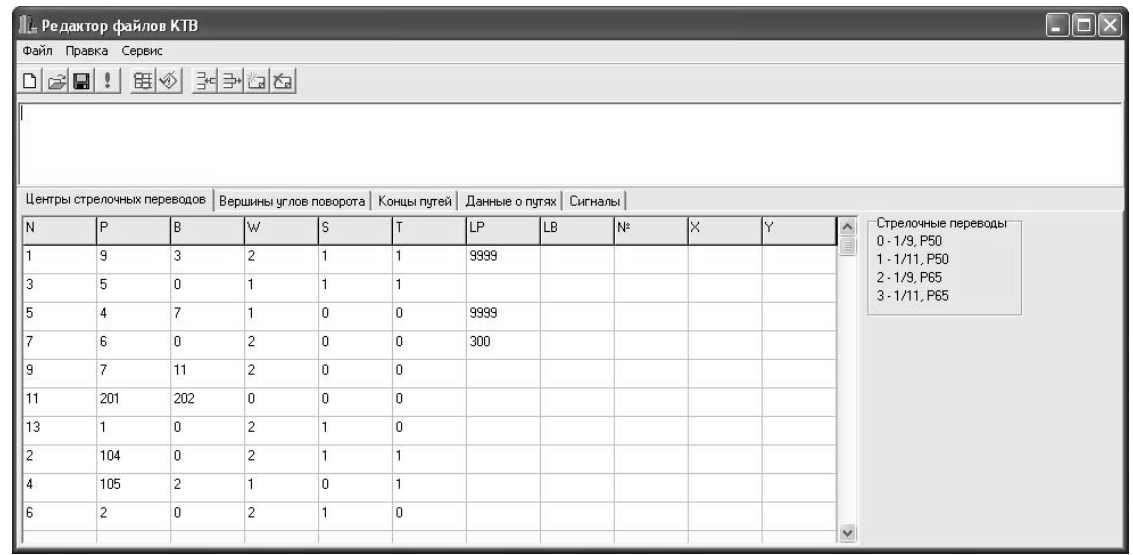

Рисунок 6.6 - Окно редактора внутренней модели путевого развития

Результатами автоматизированного расчета являются таблицы кривых, расстояний между точками и координат. Вывести эти таблицы в окно «Сообщения» можно с помощью команд меню Результаты (см. Приложение В). 
На основании данных расчетов программа SaprConv.exe формирует пакетный файл чертежа AutoCAD в формате scr. Для формирования пакета необходимо нажать кнопку масштаб изображения равны, формируется чертеж путевого развития, иначе формируется двухмасштабная схема для ТРА станции. Пример масштабного плана приведен на рис. 6.7.

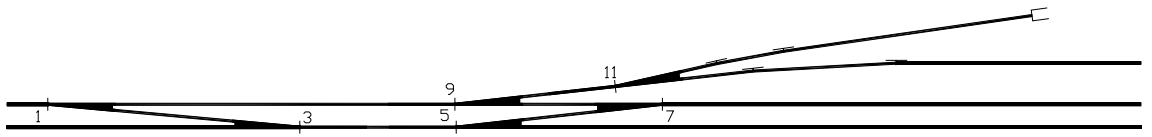

Рисунок 6.7 - Фрагмент горловины станции, полученный на основании автоматизированного расчета

При работе с программой можно копировать фрагмент изображения в буфер обмена. С этой целью необходимо мышью выделить фрагмент и выполнить команду Правка $\rightarrow$ Копировать, или нажать кнопку 㖒. 


\section{ВЫВОДЫ}

В данной монографии представлено решение актуальной научнотехнической проблемы создания интегрированной системы геометрических моделей железнодорожных станций для автоматизации синтеза планов их путевого развития.

Основные научные результаты и выводы работы заключаются в следующем:

1. Выполненный анализ научных работ по проблеме автоматизации проектирования железнодорожных станций показал, что методы прямого синтеза оптимальной конструкции планов путевого развития практически отсутствуют. В связи с этим система моделей должна поддерживать итерационный процесс совершенствования исходного варианта станции на основе последовательного многократного решения задач анализа и синтеза. Для реализации данного подхода разработана методика автоматизированного преобразования немасштабной схемы станции, разработанной проектировщиком, в масштабный план. При этом обеспечивается возможность интерактивного ввода, обработки и вывода информации о путевом развитии станций в графической форме.

2. Разработана система структурно-параметрических моделей станций (входная, внутренняя, выходная) а также алгоритмов выполнения проектных процедур, которые позволяют автоматизировать процесс синтеза путевого развития станций на всех его этапах. В основу моделей положено представление схем станций в виде ориентированных графов.

3. В качестве входной модели использован орграф, представляемый списком графических примитивов, каждый из которых представляется ассоциативным списком. Указанная модель позволяет выполнить графический ввод и редактирование схемы, а также автоматизировать построение внутренней модели станции. Графический ввод схем, дополненный вводом отдельных числовых параметров, позволяет существенно ускорить процесс синтеза за счет ликвидации этапа ручного кодирования. 
4. Для графического ввода схем адаптирован универсальный графический пакет AutoCAD, использование которого позволяет уменьшить затраты на разработку программного обеспечения и на обучение пользователей.

5. Внутренняя модель станции включает каноническую модель и модель горизонтальных путей станции. Каноническая модель станции построена на основе взвешенного орграфа, для представления которого использованы списки инцидентности, являющихся наиболее компактной формой записи орграфов рассматриваемого типа. Данная модель позволяет выполнить анализ схемы, автоматическое распознавание типовых элементов и их расчет с использованием соответствующих алгоритмов.

Разработана модель горизонтальных путей станции, основанная на использовании древовидного графа, позволяющая автоматически определять пары путей, для которых должна быть задана ширина междупутья.

6. Разработана методика расчета основных параметров плана станции (прямых вставок, координат, сопрягающих кривых). Для контроля наличия в схеме замкнутых контуров в процессе расчета осуществляется построение остовного дерева на орграфе схемы.

7. В качестве канонической модели горочных горловин использовано взвешенное ориентированное бинарное дерево; на базе данной модели разработан итерационный метод расчета углов поворота дополнительных кривых.

8. При проектировании конечных соединений сортировочных путей, реализован интерактивный метод, обеспечивающий изменение параметров путей с одновременной графической визуализацией его результатов и контролем точек, определяющих места возможной установки замедлителей парковых тормозных позиций. Указанные точки находятся в местах пересечения эквидистант смежных путей, построение которых осуществляется с использованием методов вычислительной геометрии.

9. Для проектировании крупных станций, состоящих из нескольких парков, а также расположенных на кривых, их разбивают на блоки, в пределах которых пути прямолинейны и горизонтальны. Каждый блок рассчиты- 
вается в локальной системе координат, после чего блоки объединяются в глобальной системе координат станции. При этом выполняется преобразование координат точек блоков и сопряжение соответствующих путей соединительными кривыми.

10. Состав и структура выходных моделей станции определяется целями решаемой прикладной задачи. Разработаны модели для построения масштабных планов и схем станций средствами AutoCAD, подготовки технической документации, организации интерактивного синтеза планов путевого развития станций сложной конструкции.

11. Выполнена программная реализация разработанных алгоритмов синтеза планов путевого развития станций, которая подтвердила их эффективность. Разработанные методы и алгоритмы могут быть использованы при создании САПР железнодорожных станций и узлов; их реализация позволит увеличить производительность труда проектировщиков, повысить качество проектирования. 


\section{БИБЛИОГРАФИЧЕСКИЙ СПИСОК}

1. Проектирование железнодорожных станций и узлов / Справ. и метод. руководство / Под ред. Козлова А.М., Гусевой К.Г. - М.: Транспорт, 1981. $592 \mathrm{c}$.

2. Савченко И.Е., Земблинов С.В., Страковский И.И. Железнодорожные станции и узлы. - М.: Транспорт, 1980. - 479 с.

3. Таль К.К., Пономарева Л.Ф. Методика расчета соединений путей на ЭВМ / Вопросы проектирования железных дорог: Сб. научн. тр. ЦНИИС. Вып. 63. - М.: Транспорт, 1967. - с. 142 - 156.

4. Мирошниченко В.М. О машинном представлении схем крупных железнодорожных станций // Сб. Кибернетика и транспорт. - М.: Наука, 1968. c. 178 - 206.

5. Расчеты соединения путей на ЭВМ / Методические указания по проектированию железнодорожных узлов и станций. - К.: Киевгипротранс, 1981. $-23 \mathrm{c}$.

6. Антонов К. В. Автоматизированные расчеты при проектировании планов парков путей на станции // Пути повышения производительности труда, сокращения сроков проектирования и строительства транспортных сооружений: Сб. научн. тр. - М.: ЦНИИС, 1986. - с. 133 - 135.

7. Мирошниченко В.М. Некоторые вопросы автоматизации проектирования железнодорожных станций с помощью ЭЦВМ: Автореф. дис... канд. техн. наук: 434/ ХИИТ. - К., 1969. - 25 с.

8. Б. дел Рио, Мирошниченко В.М. Определение на ЭЦВМ пропускной способности станций по горловинам // Сб. Кибернетика и транспорт. - М.: Наука, 1968. - с. 207 - 231.

9. Акулиничев В.М., Бодюл В.И., Голубев В.В. Проблемы автоматизации проектирования ж.д. станций и узлов // Вопросы проектирования и технология транспортных узлов: Межвуз. сб. научн. тр. - Вып. 674. - М.: МИИТ, 1980. - с. 3 - 9. 
10. Акулиничев В.М. Алаев М.М. Основы автоматизации проектирования железнодорожных станций и узлов: Межвуз. сб. научн. тр. - Вып.735. М.: МИИТ, 1983. - с. 3 - 14.

11. Железнодорожные станции и узлы промышленного транспорта: Учебник для ВУЗов / В.М. Акулиничев, Л.П. Колодий, Н.Г. Мищенко, В.А. Сидяков / Под ред. В.М. Акулиничева. - М.: Транспорт, 1986. - 352 с.

12. Алаев М.М. Формализация элементов схем станций для расчетов на ЭВМ. // Проблемы наращивания мощности станций и узлов: Межвуз. сб. научн. тр. - Вып. 765. - М.: МИИТ, 1985. - с. 58 - 59.

13. Олейников Л.М., Алаев М.М. Оптимизация проектных решений элементов схем станций с помощью ЭВМ // Тезисы докладов IX областной научно - практической конференции. - Куйбышев: КИИТ. - 1985. - с. 14 - 15.

14. Олейников А.М., Алаев М.М., Корешков В.Н. Основные принципы проектирования схем станций с помощью ЭВМ. // Тезисы докладов IX областной научно - практической конференции. - Куйбышев: КИИТ. - 1985. - с. 15 -16 .

15. Корешков В.Н. Способ представления схемы сортировочной станции в ЭВМ // Проблемы перспективного развития железнодорожных станций и узлов: Межвуз. сб. научн. тр. - Гомель: БелИИЖТ, 1985. - с. 91 - 96.

16. Чернов В.Н., Пивоваров В.С. Формализация схем железнодорожных станций для графического отображения на ЭВМ. // Вопросы совершенствования системы автоматизированного проектирования железнодорожных станций и узлов: Межвуз. сб. научн. тр. - Вып. 214/54. - Ташкент: ТашИИТ, 1989. - c. 8 - 13.

17. Методические указания по проектированию железнодорожных узлов и станций. - Киев: Киевгипротранс, Вып. 100, 1985 - 32с.

18. Томилина Г.С. Схемы горловин участковых станций с минимальными затратами на ремонт стрелочных переводов и подвижного состава // Проблемы перспективного развития железнодорожных станций и узлов: Межвуз. сб. научн. тр. - Гомель: БелИИЖТ, 1987. - с. 74 - 78. 
19. Томилина Г.С. Необходимые условия для автоматизации проектирования станций. // Вопросы совершенствования системы автоматизированного проектирования железнодорожных станций и узлов: Межвуз. сб. научн. тр. - Вып. 214/54. - Ташкент: ТашИИТ, 1989. - с. 29 - 30.

20. Родимов Б.А., Павлов В.Е., Прокинова В.Д. Проектирование механизированных и автоматизированных сортировочных горок. - М.: Транспорт, 1980. $-96 \mathrm{c}$.

21. Бузанов С.П., Карпов А.М., Рыцарев М.А. Проектирование механизированных и автоматизированных сортировочных устройств. М.: Транспорт, 1965.-232c.

22. Павлов В.Е. Элементы оптимального проектирования плана горловины автоматизированной сортировочной горки. // Железнодорожные системы автоматики и телемеханики с применением бесконтактных элементов: Сб. научн. тр. ЛИИЖТа. - Вып. 314. - Л.: Транспорт, 1971. - с. 148 - 155.

23. Бобровский В.И., Козаченко Д.Н. Интегрированные модели железнодорожных станций // Інформаційно - керуючі системи на залізничному транспорті. - 2002. - № 4, 5 (додаток). - с. 23.

24. Кристофидис Н. Теория графов. Алгоритмический подход. - М.: Мир, 1978.

25. Липский В. Комбинаторика для программистов. - М.: Мир, 1988. $213 \mathrm{c}$.

26. Бобровский В.И. Структурные модели путевого развития железнодорожных станций для автоматизированного проектирования // Информационно - управляющие системы на железнодорожном транспорте. - 1997. - №3. - c. $58-63$.

27. Головнич А.К. Автоматизация проектирования железнодорожных станций. - Гомель.: БелГУТ. 2001, - 202 с.

28. Майкл Ласло. Вычислительная геометрия и компьютерная графика на С++. - М.: Издательство БИНОМ, 1997. - 304 с. 
29. Фоли Дж., вэн Дэм А. Основы интерактивной машинной графики: В 2 - х книгах. Кн. 1. - М.: Мир, 1985. - 368 с.

30. Муха Ю.А., Бобровский В.И. Использование ЭВМ при расчете плана горочной горловины сортировочного парка // Применение вычислительной техники в учебном процессе: Труды вузов МПС. - Вып. 591. - М.: МИИТ. - 1977. - с. 140 - 147.

31. Бобровский В.И. Автоматизация проектирования стрелочных горловин сортировочных парков // Совершенствование технических устройств и технологии управления процессом расформирования составов на сортировочных горках: Межвуз. сб. научн. тр. - Днепропетровск: ДИИТ, 1986. - с. 42 $-49$.

32. Правила и нормы проектирования сортировочных устройств на железных дорогах Союза ССР. ВСН 207 - 89. - М.: Транспорт, 1992. - 104 с.

33. Горелик А.Г. Автоматизация инженерно - графических работ с помощью ЭВМ. - Мн.: Вышейшая школа. 1980, - 208 с.

34. Конструкции и параметры стрелочных горловин автоматизированных и механизированных сортировочных горок: Альбом схем. - Л.: Гипротранссигналсвязь, 1983. - 75 с 
ПРИЛОЖЕНИЯ 


\section{ПРИЛОЖЕНИЕ А РАСЧЕТ ПЛАНА ПУТЕВОГО РАЗВИТИЯ СТАНЦИИ}

В данном приложении приведены входная и внутренняя модели станции, показанной на рис. 2.3 (см. п. 2.2.1), а также результаты расчета плана путевого развития.

В табл. А.1 приведена входная модель в виде текстового файла в формате *.stn (см. п. 2.1); в табл. А.2 - внутренняя модель, состоящая из канонической модели и модели горизонтальных путей, в виде текстовых файлов KTВ и PUT.

Результаты расчета плана путевого развития станции (элементы кривых, координаты точек и расстояния между ними) приведены в табл. А.2. На рис. А.1 приведен масштабный план станции, полученный с помощью программы SaprConv.exe. 


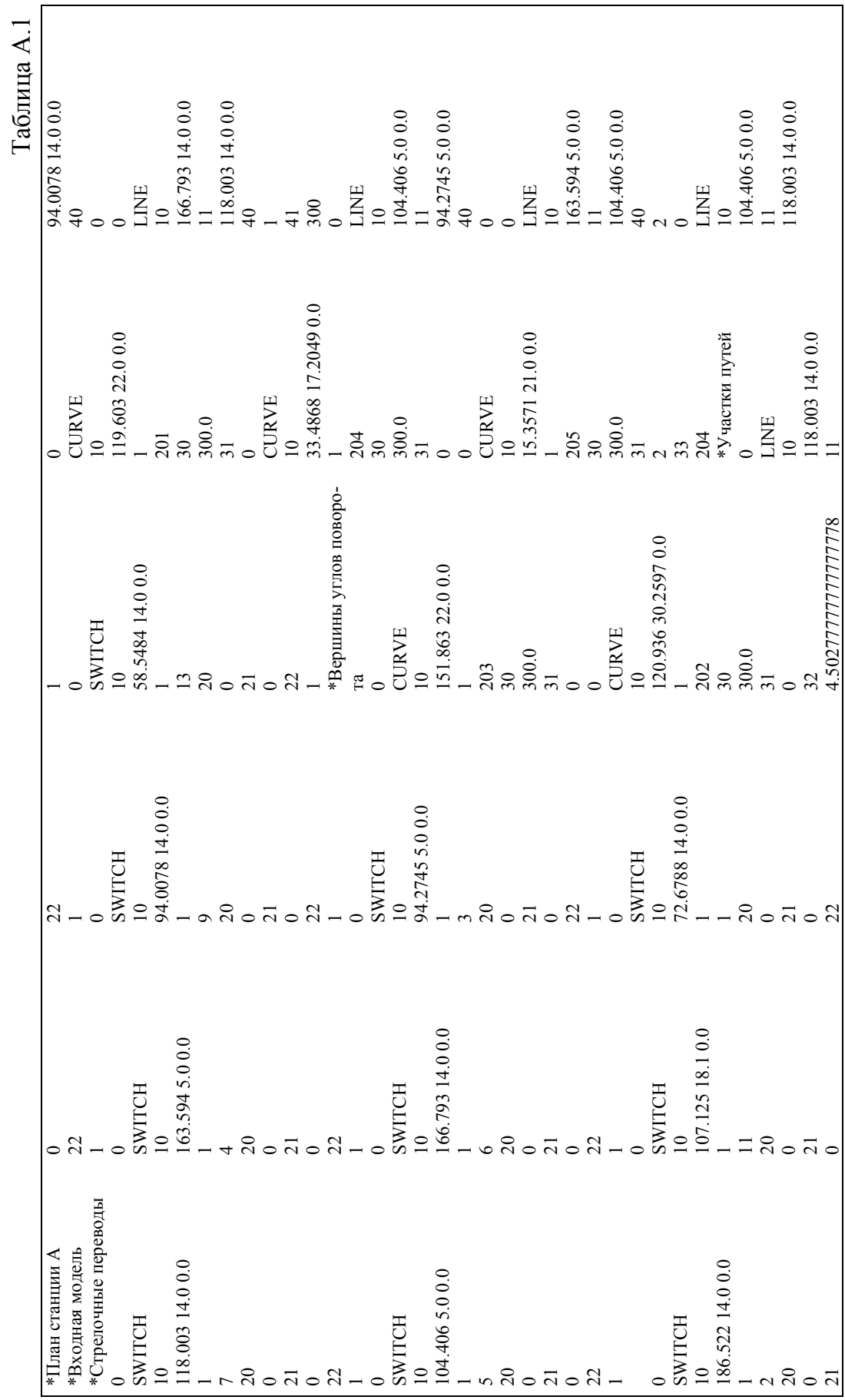




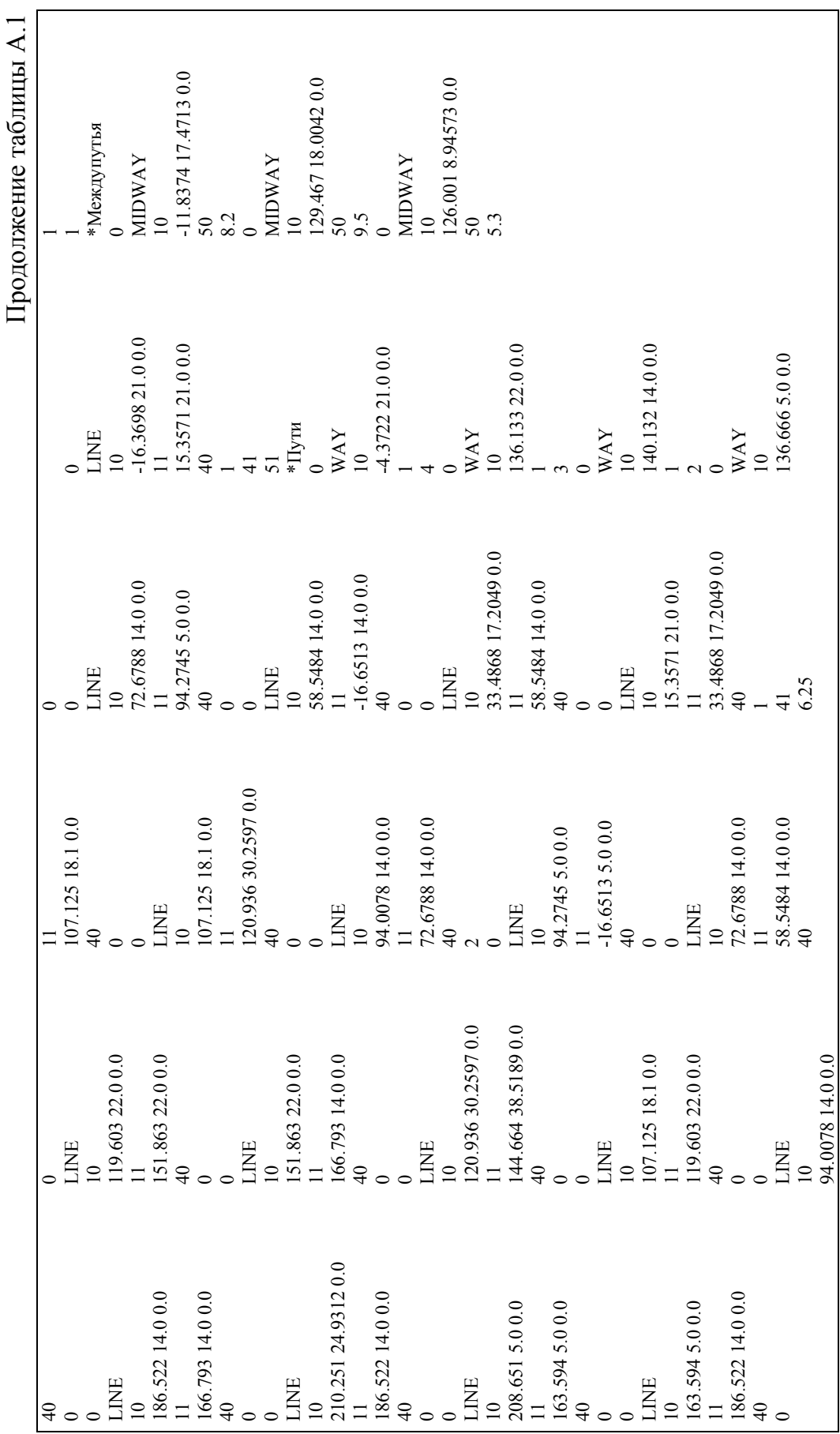




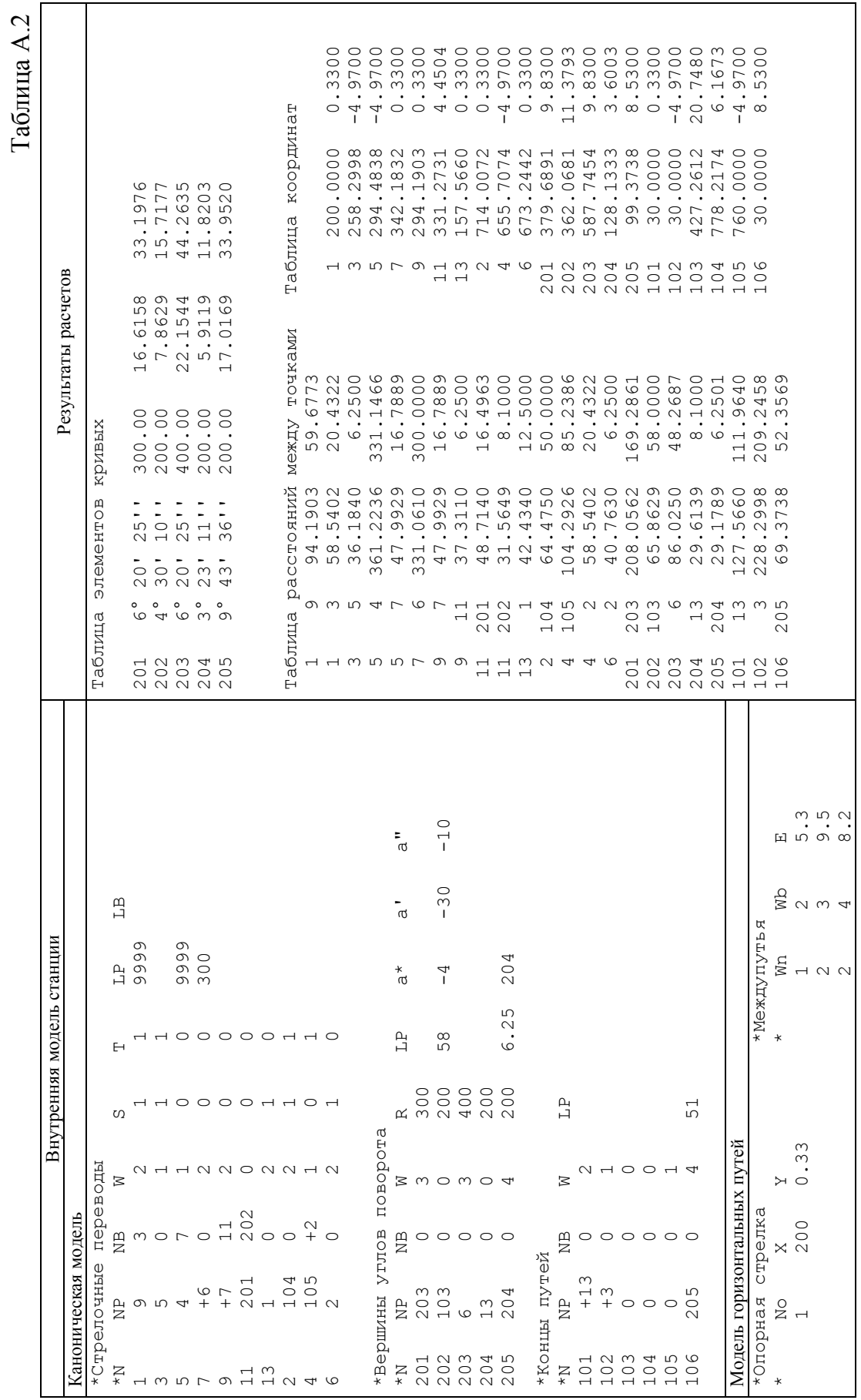




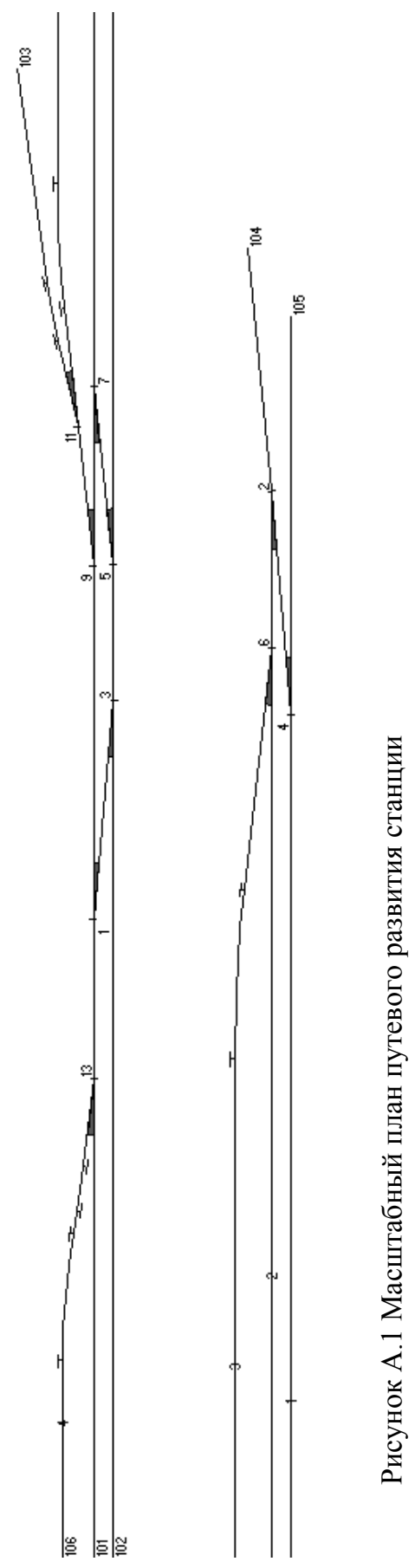




\section{ПРИЛОЖЕНИЕ Б \\ РАСЧЕТ ПЛАНА ГОРОЧНОЙ ГОРЛОВИНЫ}

В приложении приведен пример автоматизированного расчета плана горочной горловины, схема которой показана на рис. 2.6 (см. п. 2.3).

В табл. Б.1 приведена внутренняя модель горочной горловины, в табл. Б.2 - результаты расчета (параметры кривых, координаты центров стрелочных переводов, координаты точек установки замедлителей, расстояния между точками плана). В качестве расчетного принят путь 6.

Далее на рис. Б.1 приведен масштабный план горочной горловины.

Таблица Б.1

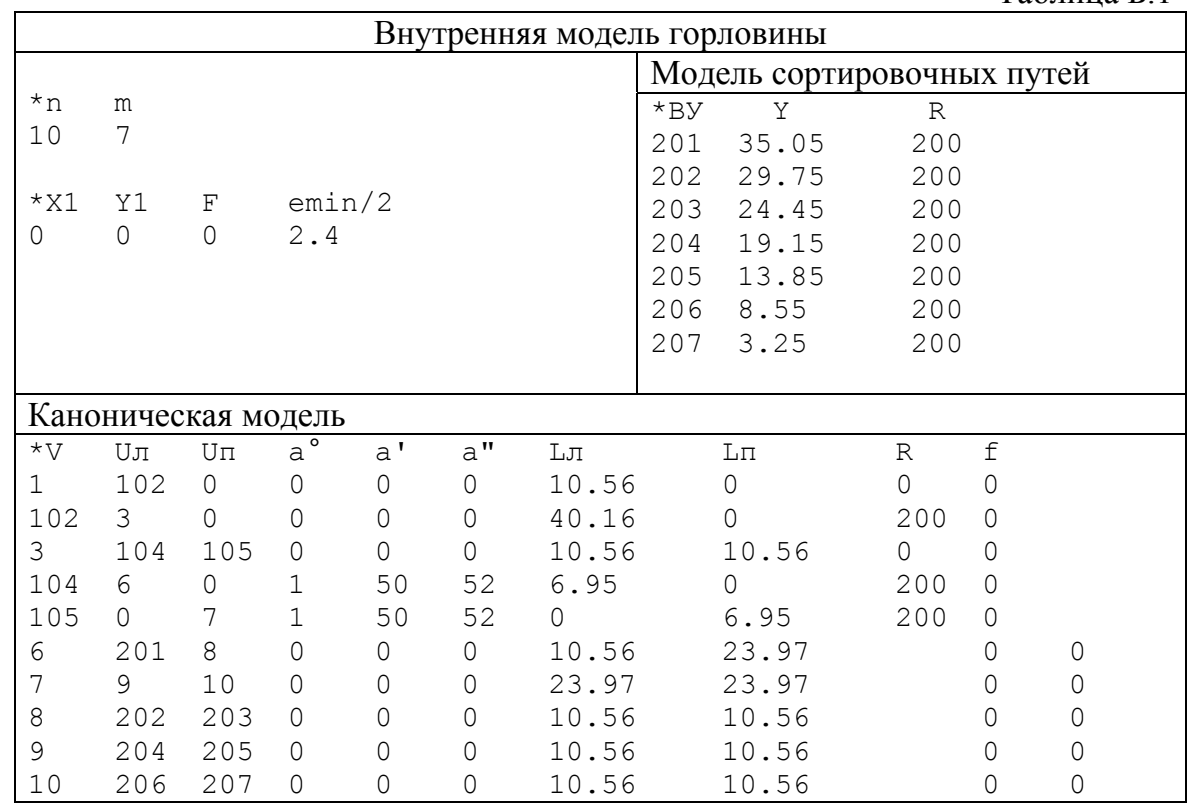


Таблица Б.2

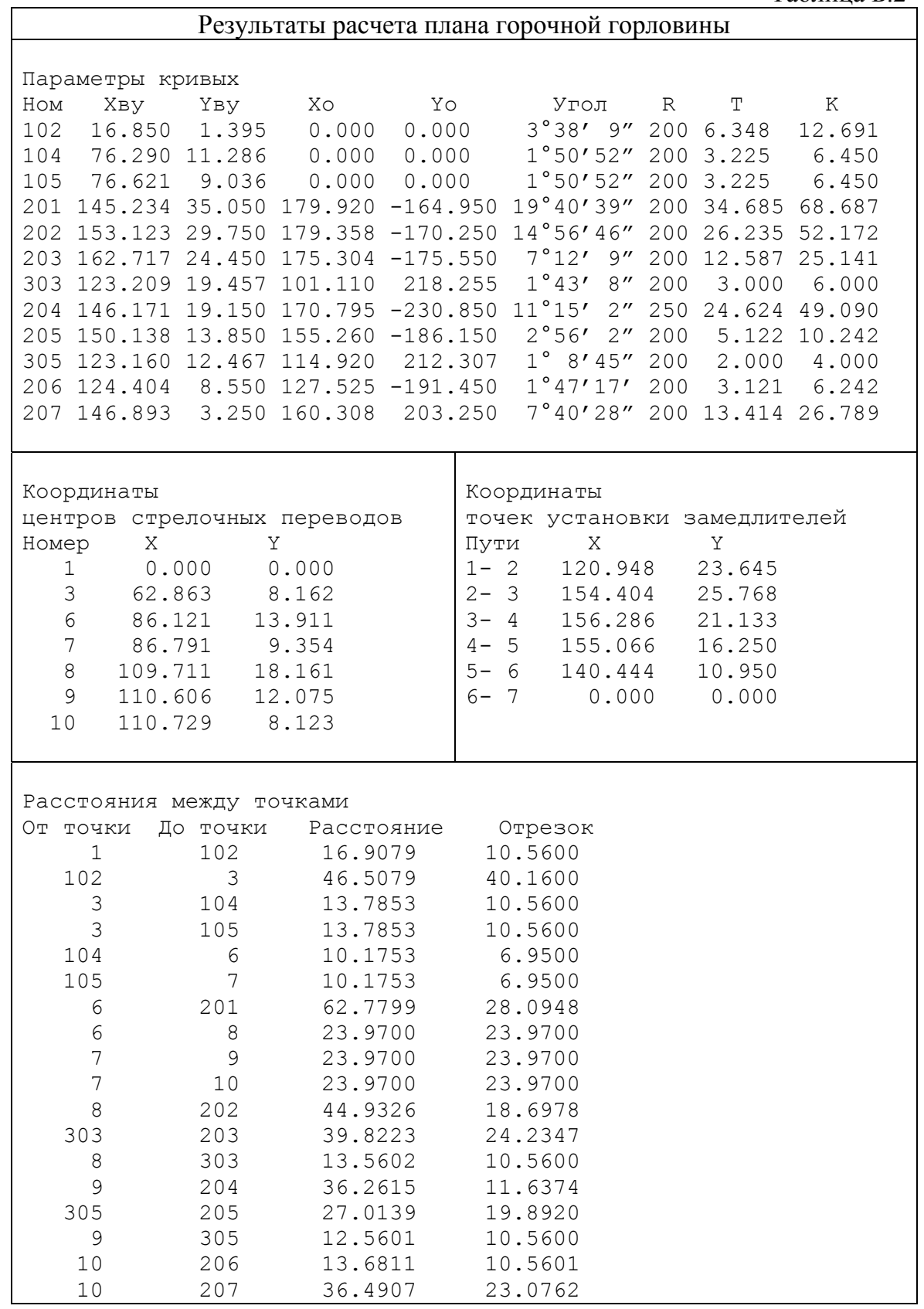




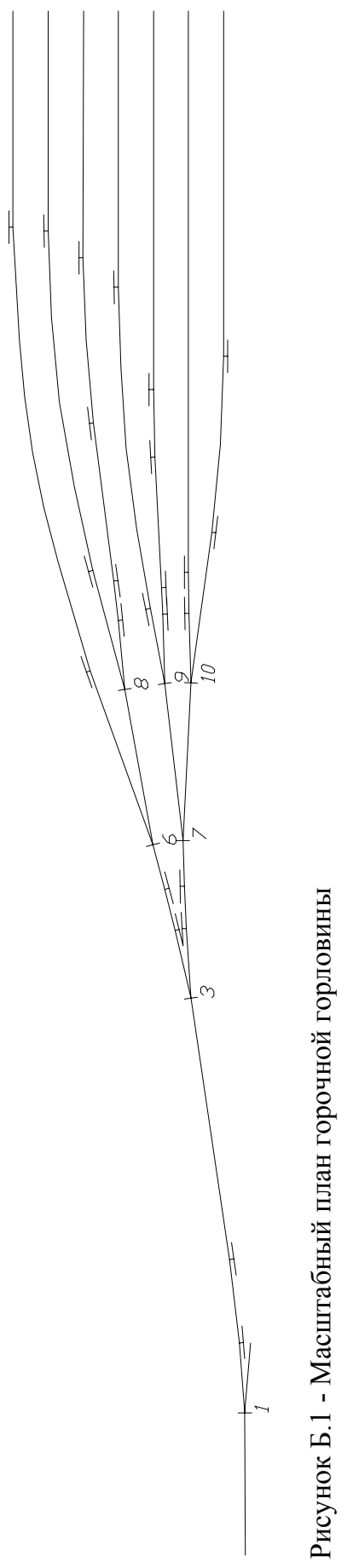




\section{ПРИЛОЖЕНИЕ В \\ РАСЧЕТ ПЛАНА СОКРАЩЕННОЙ ГОРЛОВИНЫ}

В табл. В.1 приложения приведена внутренняя модель сокращенной стрелочной горловины, схема которой показана на рис. 4.6 (см. п. 4.4). В данной горловине для сокращения ее длины уложена дополнительная кривая 201 с углом поворота $\beta$ и обратные ей кривые 203-207, соответственно, на путях 3-7. Для задания расчетного пути в канонической модели горловины в строке вершины 201 вместо неизвестного значения угла $\alpha$ указан номер конечной вершины расчетного пути (207).

В табл. В.1 также приведены результаты расчета плана сокращенной горловины: параметры кривых, координаты точек и расстояния между ними.

На рис. В.1 приведен масштабный план сокращенной горловины, полученный с помощью программы SaprConv.exe. 


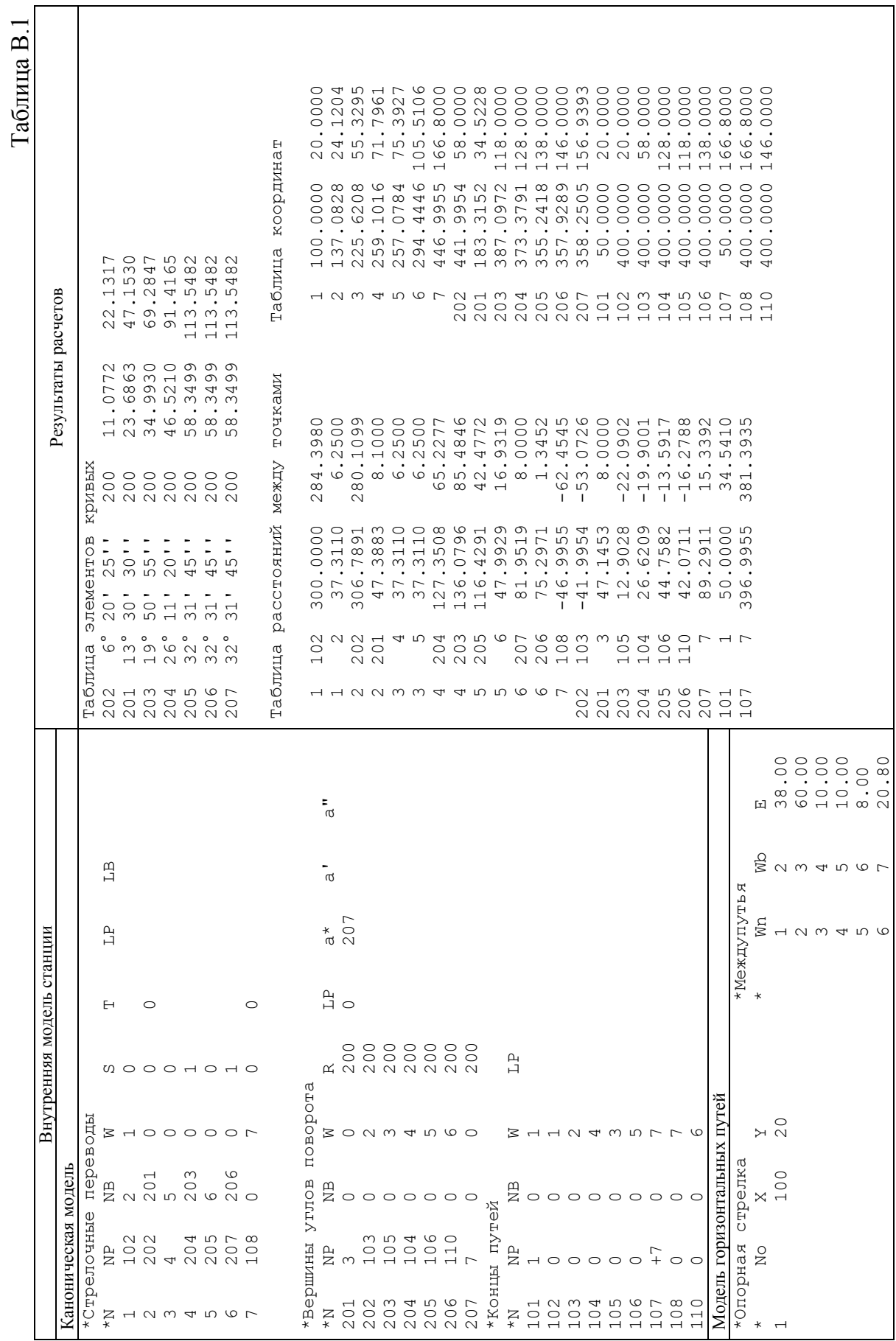




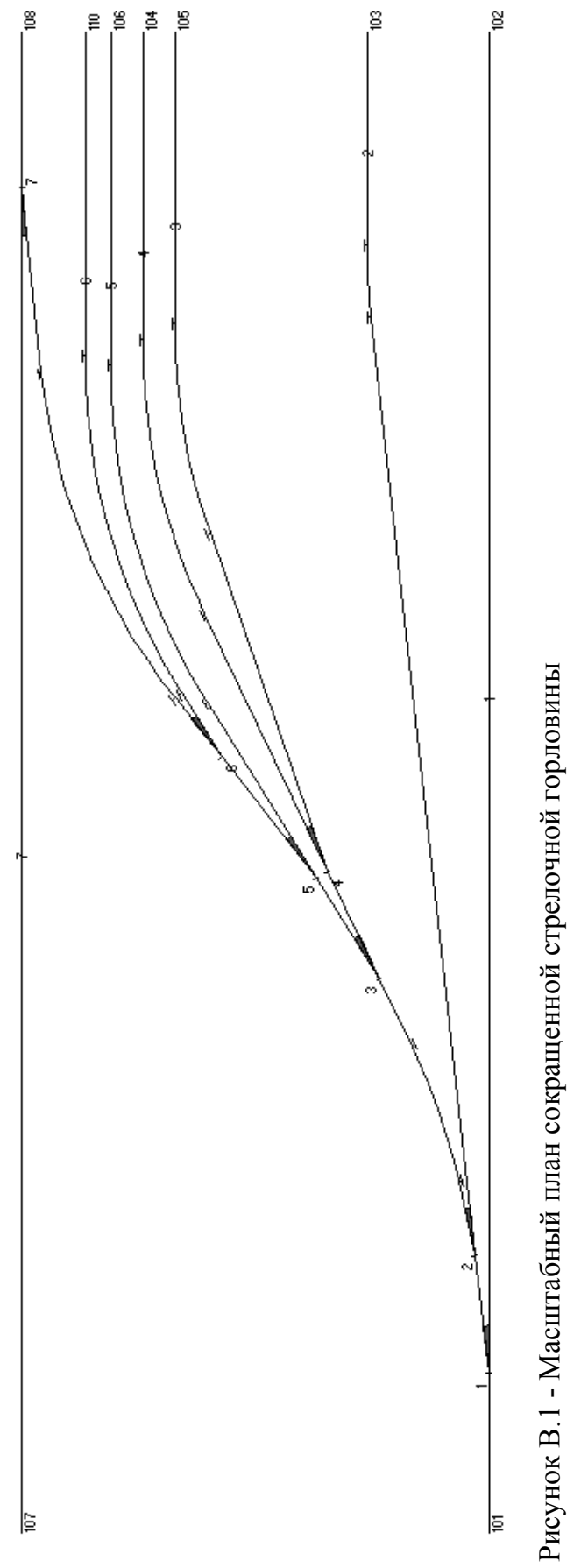




\section{ПРИЛОЖЕНИЕ Г}

\section{РАСЧЕТ ПЛАНОВ СТРЕЛОЧНЫХ УЛИЦ}

В данном приложении приведены внутренние модели стрелочных улиц, рассмотренных в п. 4.5, а также результаты их расчета и масштабные планы, полученные с помощью программы SaprConv.exe.

Таблица Г.1 - Сокращенная улица с увеличенным 1-м междупутьем (7,6115 м), равным расчетному (минимальному); другие междупутья одинаковые. Для кривой с углом $\beta$ (201) в канонической модели задана расчетная вершина (202) для его определения.

Таблица Г.2 - Сокращенная улица с увеличенным 1-м междупутьем (10 м), которое больше расчетного (минимального); другие междупутья одинаковые. Для кривой с углом $\beta$ (201) в канонической модели задана его величина (149'34.2"), pacсчитанная при $d=6.25$ м и $e=5.3$ м.

Таблица Г.3 - Сокращенная улица с увеличенным 1-м междупутьем (6.5 м), которое меньше расчетного (минимального); другие междупутья одинаковые. Для кривой с углом $\beta$ (201) в канонической модели задана расчетная вершина (202) для его определения. Неизвестные вставки 2-3, 3-4 должны быть определены в результате расчета, с этой целью они помечены кодом 9999.

Кроме того, в приложении приведены внутренние модели, результаты расчета и масштабные планы: стрелочной улицы под двойным углом крестовины (табл. Г.4), концентрической веерной улицы с одинаковыми междупутьями (табл. Г.5), неконцентрической веерной улицы с одинаковыми междупутьями (табл. Г.6). 


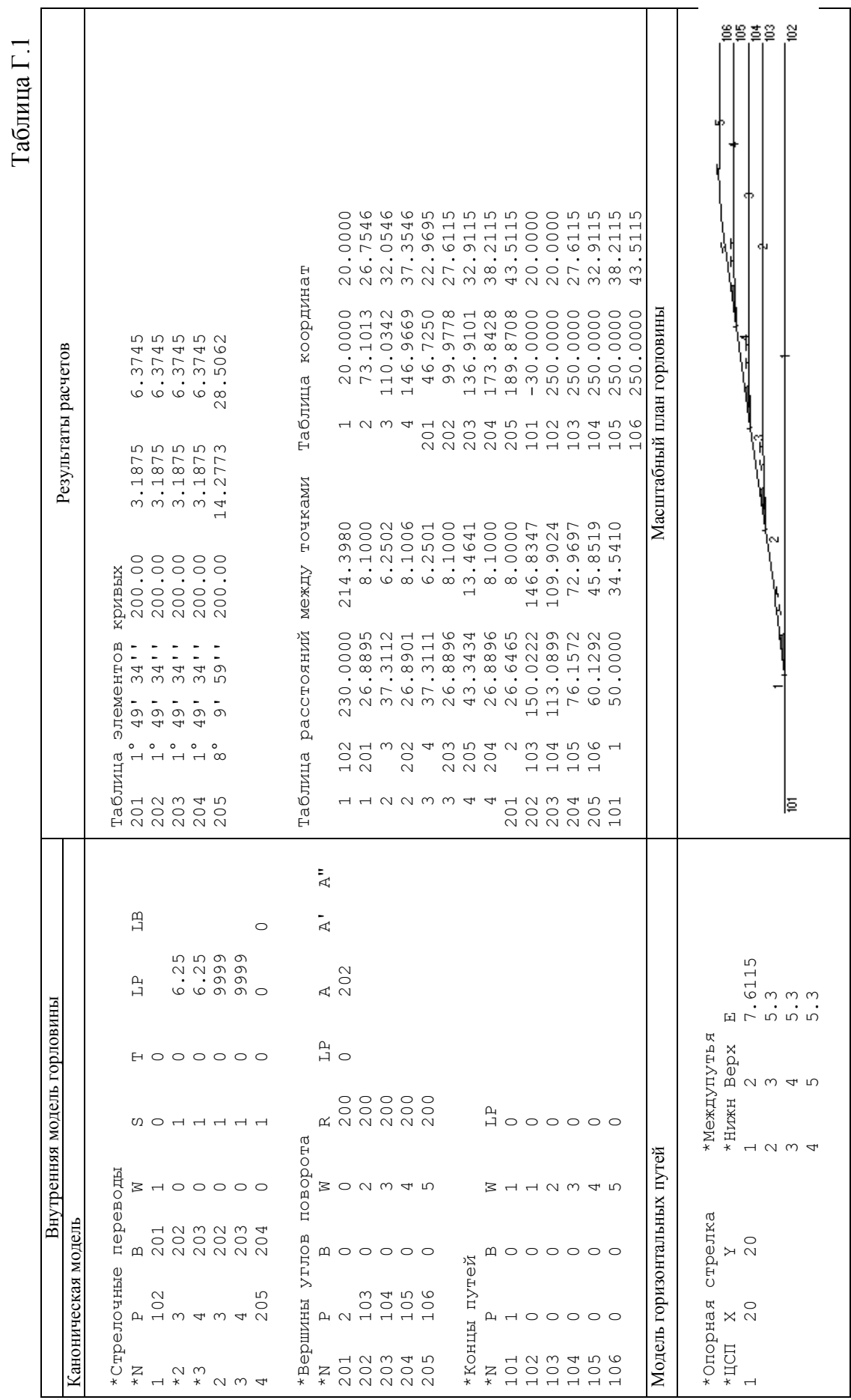




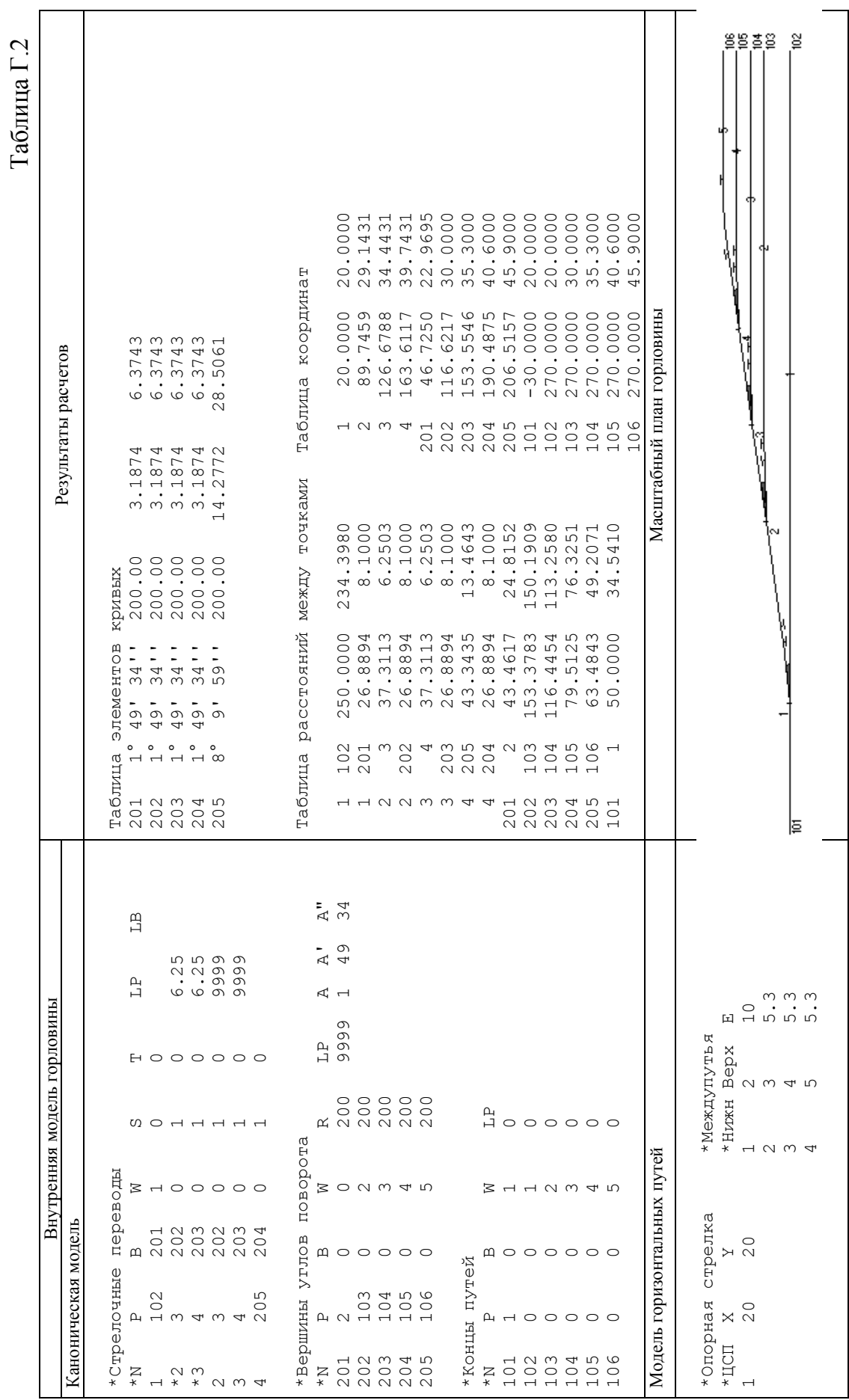




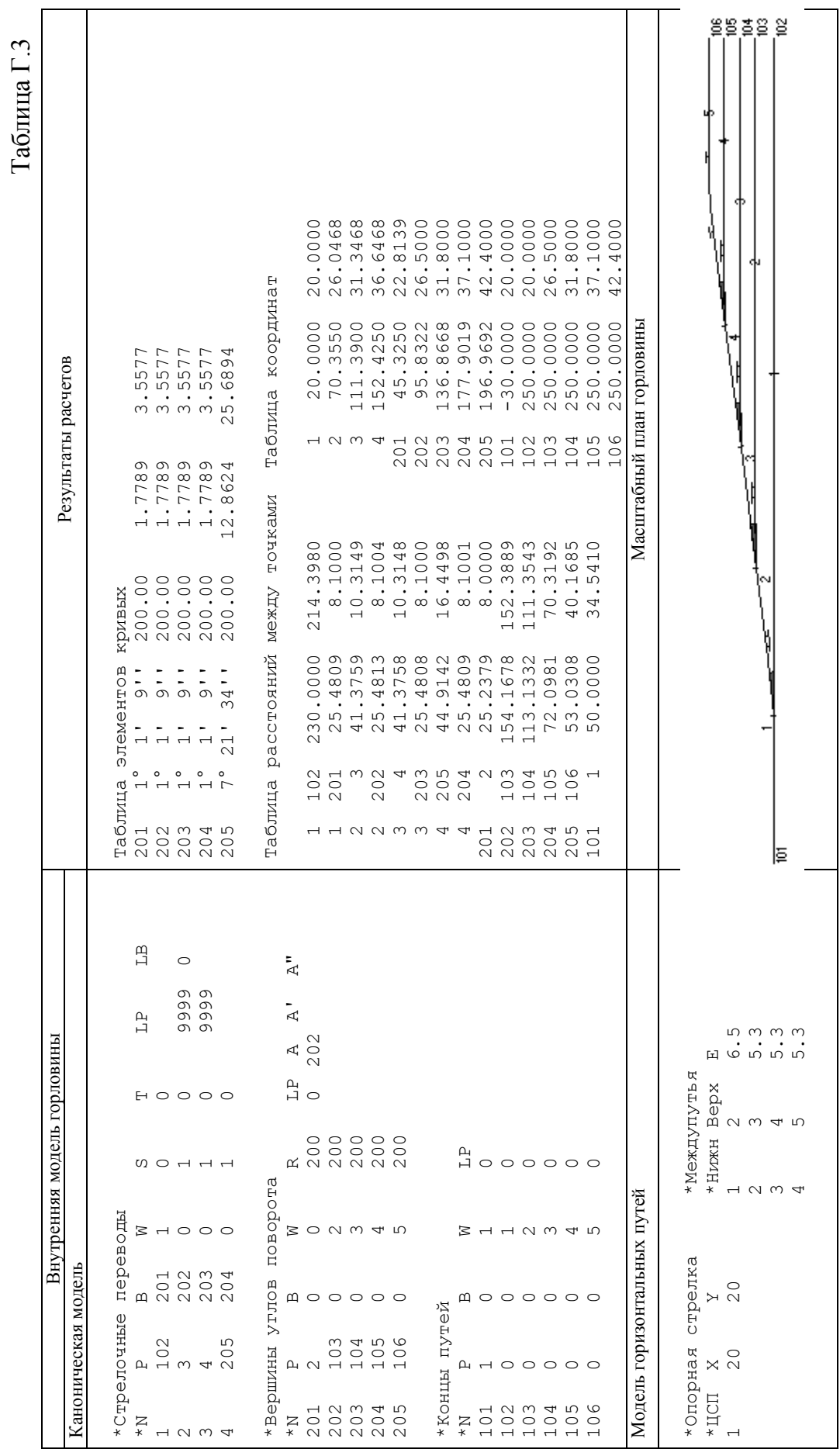




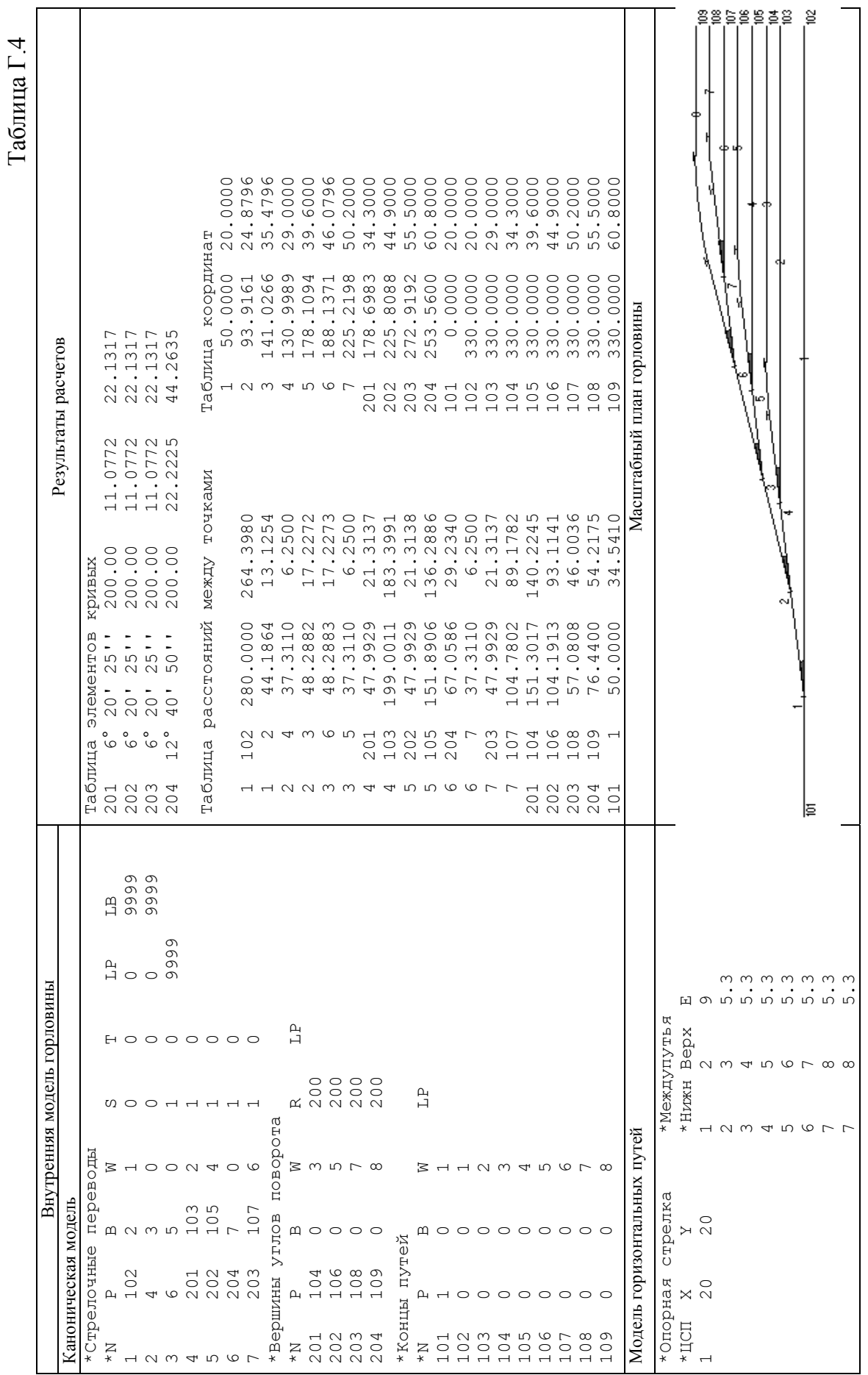




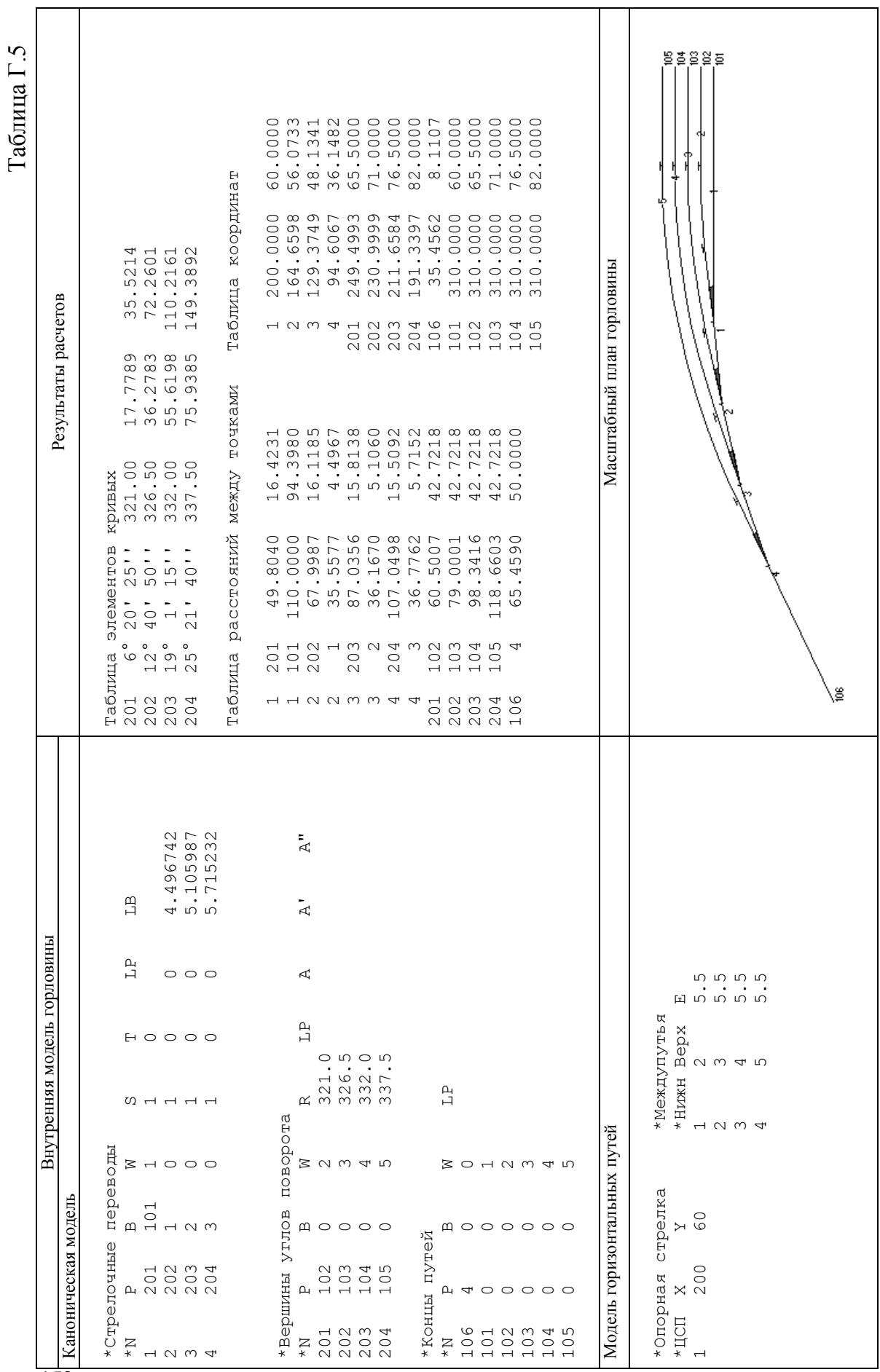


응

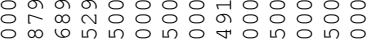

$v \sim \infty$ न ने क 긴

:

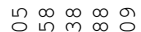

$6+1 m$ in 0

$-6 \mathrm{~m} \sim$

营 $\dot{m} \dot{\circ}$

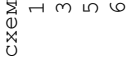

x:८:

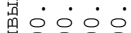
일 $\mathrm{m} \stackrel{m}{m}$

. . . . . . . . . .

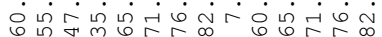

ำฟ

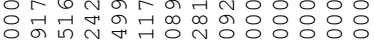
$\dot{0} \dot{0} \cdot \dot{0} \cdot \cdot \cdot \dot{0} \dot{0} \dot{0}$

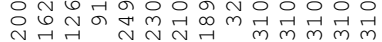

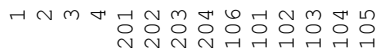

m $=z=$

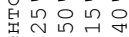

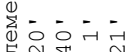
त) ซึํํㄱำ

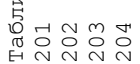

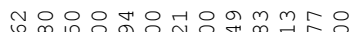

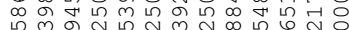
$\dot{r} \dot{\sigma} \dot{\omega} \dot{\omega} \dot{\omega} \dot{\varphi} \dot{\omega} \dot{\sigma} \dot{0}$ \%

装

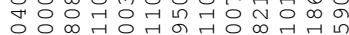
$\infty \circ \infty$ m न म न

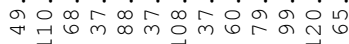
胥

\section{疍}

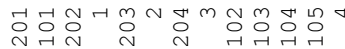

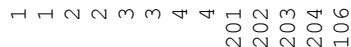

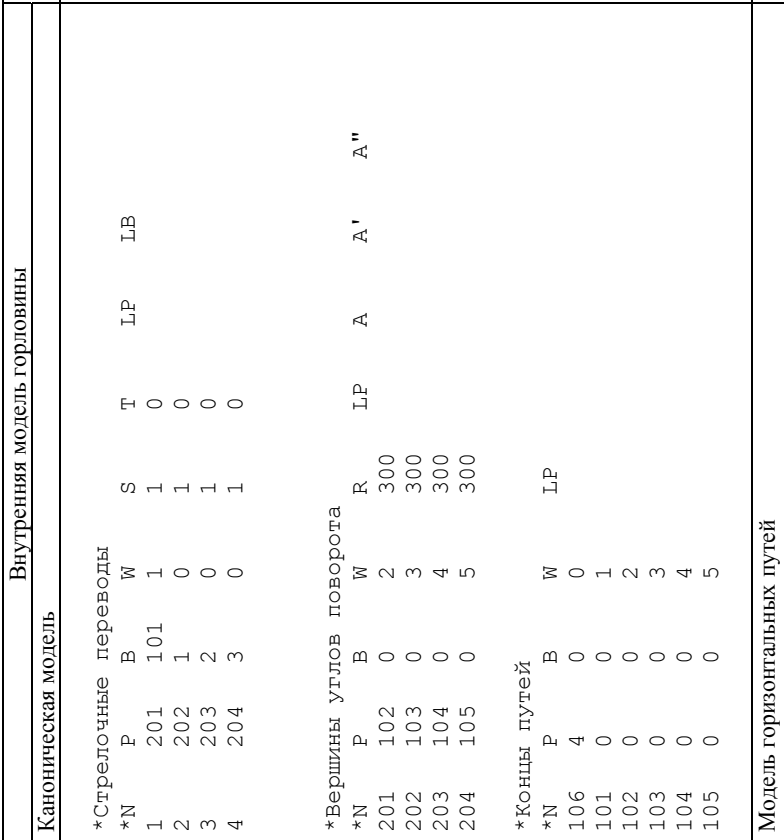

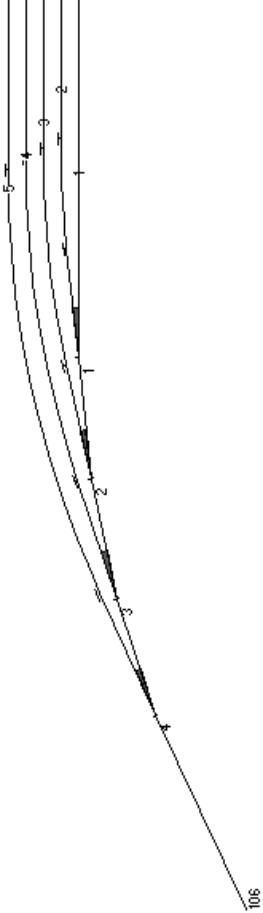

เก เก เก เก प्र ம் ம்

药

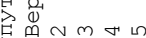

泾
先

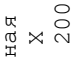
号品 
ДЛЯ ЗАМЕТОК 
ДЛЯ ЗАМЕТОК 
В. И. БОБРОВСКИЙ, Д. Н. КОЗАЧЕНКО, Р. В. ВЕРНИГОРА,

В. В. МАЛАШКИН

\title{
МОДЕЛИ, МЕТОДЫ И АЛГОРИТМЫ АВТОМАТИЗИРОВАННОГО ПРОЕКТИРОВАНИЯ ЖЕЛЕЗНОДОРОЖНЫХ СТАНЦИЙ
}

\author{
Монография \\ (на русском языке)
}

Редактор С. В. Мямлин

Компьютерная верстка $A$. А. Заиченко

Дизайн обложки $A$. A. Заиченко

Компьютерный набор А. А. Заиченко

Издательство Маковецкий Юрий Вадимович

Свидетельство ДК № 2665 от 25.10.2006 г.

49000, Украина, г. Днепропетровск, ул. Плеханова, 16, к.14

Тел. (056) 798-33-64, факс (0562) 36-79-93

e-mail: europress@gala.net

Отпечатано:

ООО фирма „Вета”. г. Днепропетровск, ул. Наб. Ленина, 9, тел. (056) 370-30-22.

Подписано в печать 18.01.10p. Формат 29,7x42 1/4. Бумага офсетная.

Печать ризограф. Усл.печ.л. 9,01. Тираж 300 экз. 